FOREST LEAF WATER CONTENT ESTIMATION USING

LiDAR AND HYPERSPECTRAL DATA 
Graduation committee:

\section{Chairman/Secretary}

Prof.dr.ir. A. Veldkamp

\section{Supervisor(s)}

Prof.dr. A. K. Skidmore

University of Twente

\section{Co-supervisor(s)}

Dr. T. Wang

University of Twente

Dr. R. Darviszadeh Varchehi University of Twente

\section{Members}

Prof.dr.ir. A. Stein

Prof.dr. F.D. van der Meer

Prof.dr. B. Höfle

University of Twente

University of Twente

Prof.dr. W. Bastiaanssen

Technical University Delft / IHE Delft

ITC dissertation number 319

ITC, P.O. Box 217, 7500 AE Enschede, The Netherlands

ISBN 978-90-365-4533-4

DOI 10.3990.1.9789036545334

Cover designed by Job Duim

Printed by ITC Printing Department

Copyright (C) 2018 by Xi Zhu

TU FACULTY OF GEO-INFORMATION SCIENCE AND EARTH OBSERVATION 


\title{
FOREST LEAF WATER CONTENT ESTIMATION USING LiDAR AND HYPERSPECTRAL DATA
}

\author{
DISSERTATION
}

to obtain

the degree of doctor at the University of Twente, on the authority of the rector magnificus, prof.dr. T.T.M. Palstra, on account of the decision of the graduation committee, to be publicly defended on Thursday 5 April 2018 at $12.45 \mathrm{hrs}$

\footnotetext{
by
}

Xi Zhu

born on 18 June 1987

in Anhui, China 
This thesis has been approved by

Prof. dr. A.K. Skidmore, supervisor

Dr. T. Wang, co-supervisor

Dr. R. Darvishzadeh Varchehi, co-supervisor 
"A journey of a thousand miles begins with a single step" Laozi 



\section{Acknowledgements}

I would like to express my sincere appreciation to those who contributed to this thesis and supported my $\mathrm{PhD}$ research in one way or the other during this memorable journey. Without their support, this work could not have reached fruition.

First and foremost, I would like to give my sincere thanks to my promoter Prof. Andrew K. Skidmore for your continuous support of my research, for your trust and motivation. I am grateful for the freedom and trust you gave me to explore on my own. Gradually I became more and more confident during my PhD. Thanks to your encouragement, I was brave enough to broaden my topic from passive remote sensing to LiDAR remote sensing, which was a completely new study area for me. Ever since I started to touch this field, I have been so passionate about it. Your insightful advice and critical comments helped me in all the time of my research and this thesis. I could not have imagined having a better promoter and mentor for my $\mathrm{PhD}$ study.

My sincere thanks go to my daily supervisor, Dr. Tiejun Wang, who has always supported me since the beginning of the $\mathrm{PhD}$ application. I am very grateful for your guidance and support during these 4 years. Especially at the beginning of my $\mathrm{PhD}$ study, your encouragement and advice helped me out deciding which research direction to pursue and proposal writing. Through the PhD study, you have always trusted and defended my research ideas and provided all the help you could to get me through the obstacles. Your comments and suggestions have always been very prompt and effective. Without your support, the papers could not have been published so smoothly. I really appreciate your academic and mental support through the rough road to finish this thesis.

Special thanks to my supervisor Dr. Roshanak Darvishzadeh for your excellent supervision. You have always been there supporting me from the proposal development to thesis writing. Your logical thinking and critical comments helped me through the $\mathrm{PhD}$ study. I am grateful for the regular meetings and discussions we shared. Whenever I had difficulties with my experiment and study, no matter if they were about facilities, experiment design or results analysis, I could always seek help from you. Your suggestions and comments were always to the point, which dramatically improved my work. I am also thankful for your kindness and emotional support during my PhD.

I would also thank Prof. K. Olaf Niemann from University of Victoria for the guidance of your knowledge about LiDAR and collaborating in my manuscripts. Bryan Hally, thank you for helping us in the Bavarian Forest National Park for fieldwork, your fieldwork and driving skills are excellent. Jing Liu, thank you for your engagement in my boring experiment and collaboration in the fieldwork. I would also like to thank Yifang Shi and Joe Premier for the help with terrestrial LiDAR data collection. Without you guys, none of the work would have been possible. I would like to thank Dr. Zhihui Wang and Dr. Abebe Mohammed Ali for providing the in situ data which were essential for the validation of my fourth paper. 
I acknowledge the German Aerospace Center (DLR) and Bavarian Forest National Park (BFNP) for providing the airborne hyperspectral and LiDAR data. In particular, I would like to thank Dr. Marco Heurich for the assistance and providing logistical facilities in my fieldwork. I would also thank Dr. Uta Heiden, Dr. Stefanie for organizing the HySpex overflight, and the provision of the pre-processed hyperspectral data.

I am thankful to ITC which provided me a nice working environment and facilities. I would like to thank Prof. Andy Nelson for the support at NRS department. My special thanks go to Dr. Boudewijn de Smeth, Dr. Caroline Lievens and Mr. Watse Siderius who helped me with my experiment in ITC Geoscience laboratory. I am grateful to Esther Hondebrink for being always so kind, supportive and helpful during my PhD. I would like to express my gratitude to Loes Colenbrander for the assistance of my $\mathrm{PhD}$ application and the support during my stay at ITC. I would also like to thank Willem Nieuwenhuis for helping me with all the technical issues. Sincere thanks to Dr. Tom Rientjes for organizing all the PhD events and ITC publication awards. I would like to thank Benno Masselink and Job Duim for the assistance in making posters and designing the thesis cover. I would also like to thank Petra Weber for being always supportive. I appreciate all the help from Theresa van den Boogaard. Special thanks to Roelof Schoppers who has always been so nice and helpful. Thanks go to all the colleagues in NRS department for their support, they are Thomas, Iris, Yousif, Henk, Louise, Anton, Wieteke, Michael and etc. Thanks to the staff members in the ITC student affairs, the ITC library, the Facility Management Service, ITC International Hotel and the Finance department for their assistance. I am very grateful to Eva Skidmore and Jackie Senior for their English editing.

I would express my sincere gratitude to my officemates and friends in Room 4-110, Dr. Elnaz Neinavaz, Maria Fernanda Buitrago, Festus Wanderi Ihwagi, Yifang Shi, and Phil Wilkes. We have had lots of memorable moments as well as profound discussions. I would also thank my fellow PhD students and colleagues in ITC: Matthew, Dimitris, Fangyuan, Ying, Nina, Sugandh, Trinidad, Haidi, Anahita, Tina, Xiaoling, Xu, Xiaolong, Junping, Zhihui, Linlin, Peiqi, Yiwen, Yijian, Luna, Yolanda, Effie, Oliver, Mitra, Yifei, Sonia, Alby, Hakan, Novi, Dewi, Riswan, Parinaz, Tawanda, Manuel, Yuhang, Hong, Ruosha, Zhenchao, Chengliang and so on.

I would like to thank my family and friends in China who have always been supportive throughout my $\mathrm{PhD}$ journey. I am most grateful to my mother and my grandparents for their unconditional love. Special thanks to Elly, Xiaoming and Caijuan who have been my best friends since college for their support of each decision I made.

Most importantly I would like to thank Roger Mulet Lázaro for being my best friend and partner. You have offered me tremendous support and love whenever I felt lost and lonely. You have always stood by me through the good times and bad. I am sincerely grateful to have you in my life. 


\section{Table of Contents}

Acknowledgements ............................................................................................................................................. i

Table of Contents............................................................................................................... iii

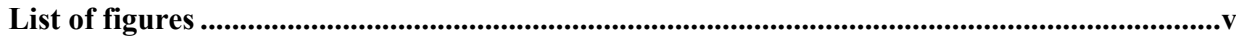

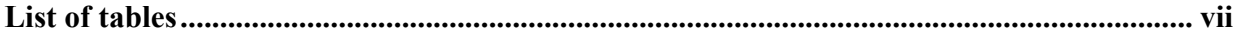

Chapter 1 Introduction ..........................................................................................................................

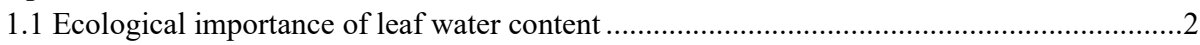

1.2 Role of remote sensing in the estimation of leaf water content .........................................

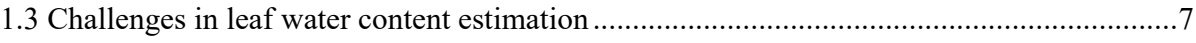

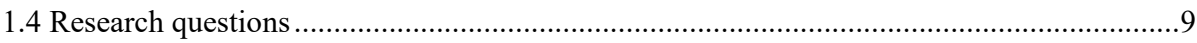

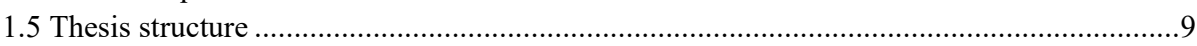

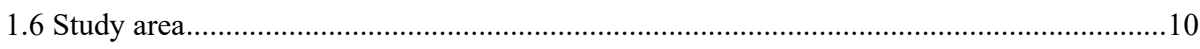

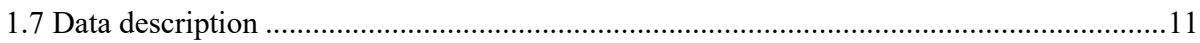

Chapter 2 3D leaf water content mapping using terrestrial laser scanner backscatter intensity

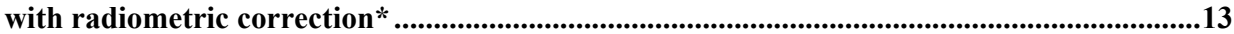

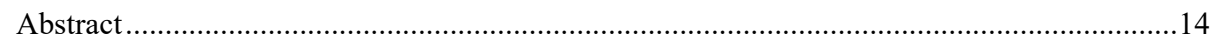

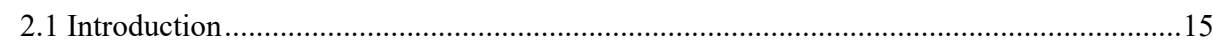

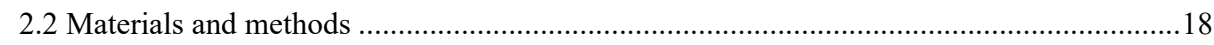

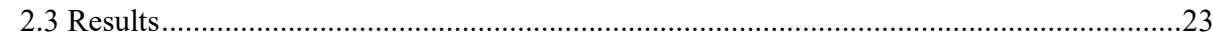

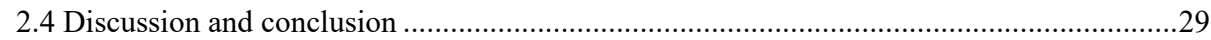

Chapter 3 Canopy leaf water content estimated using terrestrial LiDAR* ............................33

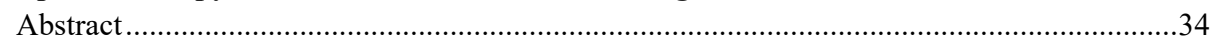

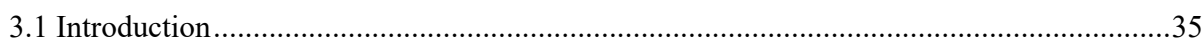

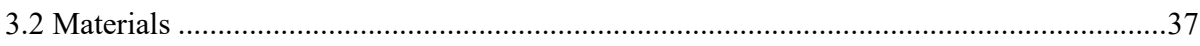

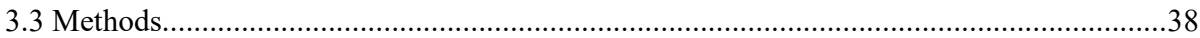

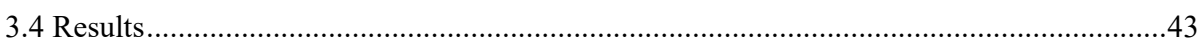

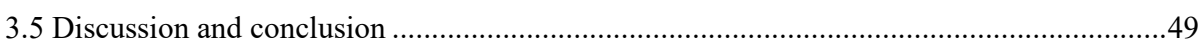

Chapter 4 Retrieval of leaf water content from airborne LiDAR and hyperspectral data* .53

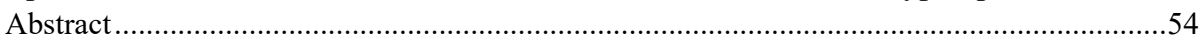

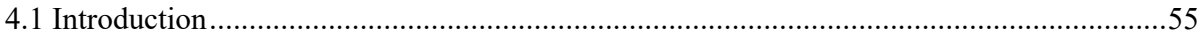

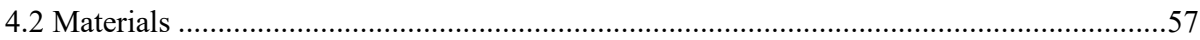

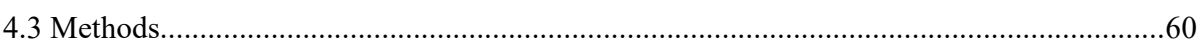

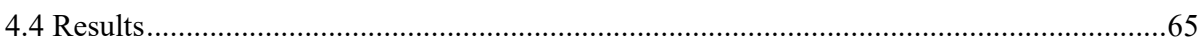

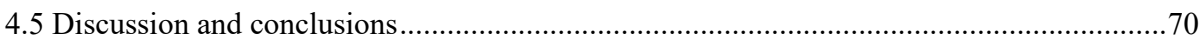

Chapter 5 Foliar and woody materials discriminated using terrestrial LiDAR in a mixed

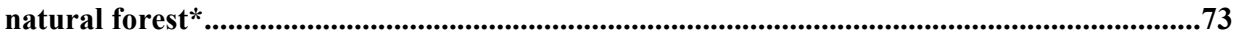

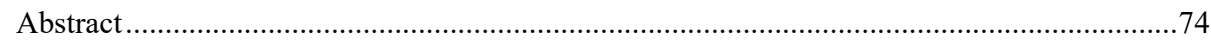

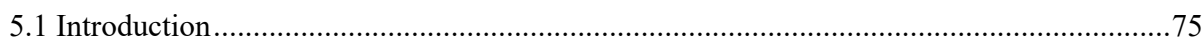

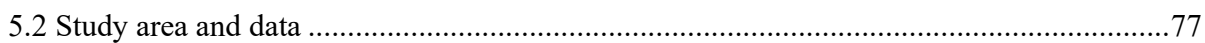

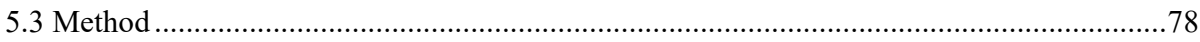

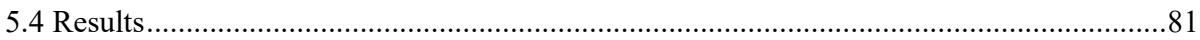

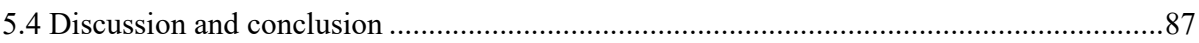

Chapter 6 Synthesis: LiDAR and hyperspectral remote sensing of leaf water content .........91

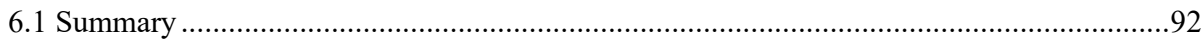

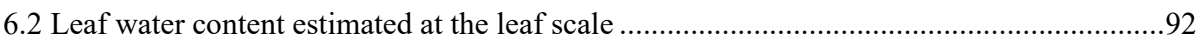

6.3 Leaf water content distribution within the canopy of individual plants..............................94 
6.4 Leaf water content estimation at the regional scale .......................................................... 98

6.5 Foliar and woody materials discriminated using terrestrial LiDAR ....................................102

6.6 Broader implication of leaf water content estimation for ecological studies .......................105

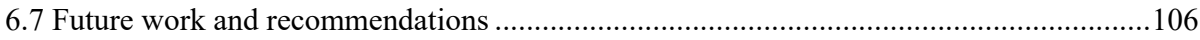

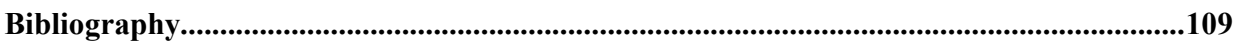

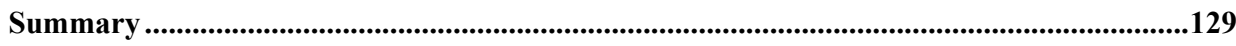

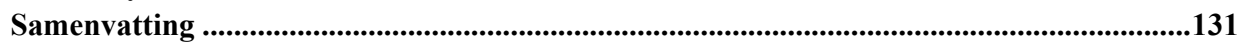

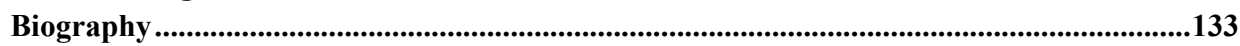

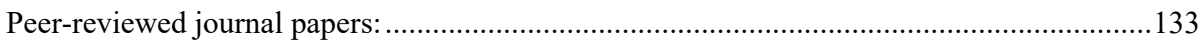

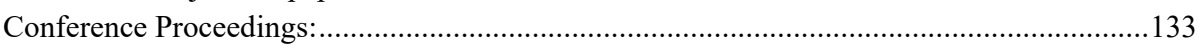

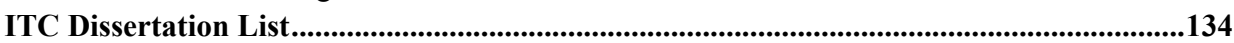




\section{List of figures}

Figure 1.1 Interaction of laser pulse with vegetation and waveform decomposition .......................5

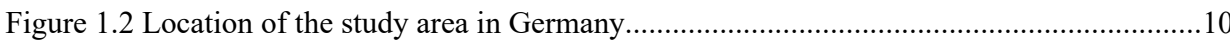

Figure 2.1 The terrestrial laser scanner measuring Spectralon panels .........................................20

Figure 2.2 Leaves of 8 plant species attached to a goniometric platform .....................................22

Figure 2.3 Surface visualization of the radiometric calibration model generated from observations

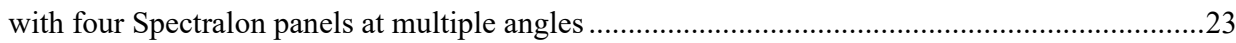

Figure 2.4 Relationships between leaf water content and backscatter intensity at a perpendicular angle. (a) before removal of specular backscatter intensity, (b) after removal of specular backscatter intensity. 24

Figure 2.5 Linear combination of the Lambertian model and Beckmann law simulation for eight plant species. Backscatter intensity at a normal incidence angle has been scaled to 1 ....................26 Figure 2.6 Terrestrial laser scanner backscatter intensity as a function of the incidence angle and reflectance for the Spectralon panels

. .27

Figure 2.7 Leaf backscatter intensity changes with incidence angle and backscatter intensity at a

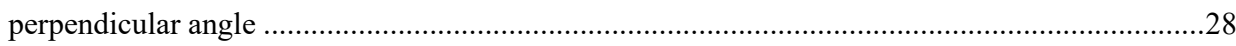
Figure 2.8 Leaf water content estimation using terrestrial laser scanner backscatter intensity before angle correction ...28

Figure 2.9 Terrestrial laser scanner backscatter intensity correction visualization. (a) before correction, (b) after correction. . .28 Figure 2.10 Leaf water content estimation using terrestrial laser scanner backscatter intensity after angle correction. (a) theoretical model based correction, (b) reference target based correction. ...29 Figure 2.11 Example of leaf water content mapping. (a) Polyscias fabian, (b) Camellia japonica.

.. .29

Figure 3.1 A waveform generated from a RIEGL VZ-400 single laser return for extended targets with high reflectance.

Figure 3.2 Waveform decomposition of a single high amplitude (a) and low amplitude (b), echo with the standard Gaussian model (G) and the modified skewed Gaussian (SG) model (the vertical lines are peak position) ...43

Figure 3.3 Waveform decomposition of a multiple echo with the standard Gaussian model (G) and the skewed modified Gaussian (SG) model (the vertical lines are peak position) ...........................4 Figure 3.4 Results of decomposition for four reflectance panels (Spectralon from Labsphere, Inc. with reflectance of $99 \%, 61 \%, 34 \%$ and $17 \%$, from left to right). (a) Gaussian decomposition, and

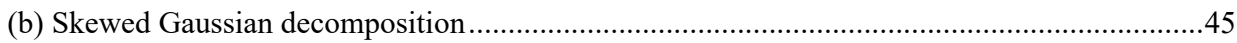
Figure 3.5 Relationships between three waveform parameters (i.e., amplitude, backscatter crosssection, and backscatter coefficient) and LWC before correcting for incidence angle effect ........46 Figure 3.6 Relationships between the backscatter coefficient and LWC after correcting for

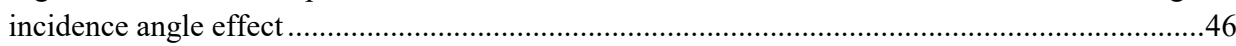
Figure 3.7 Scatterplots of the measured and estimated leaf water content by the backscatter coefficient. (a) prediction without echo width and (b) prediction with echo width ........................47 Figure 3.8 Comparison of measured and predicted leaf water content vertical profile (dwarf

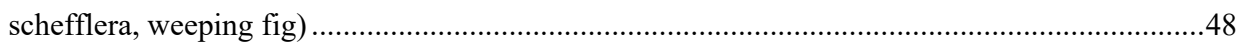
Figure 3.9 Examples of leaf water content distribution of the 2 plant species (weeping fig and ficus). (a) LWC vertical distribution (The red square indicates the mean value and the blue rectangle indicates the standard deviation) and (b) LWC visualization (plants were scanned downward from the TLS).. 
Figure 4.1 The location of the study area in Germany and the distribution of sample plots in the

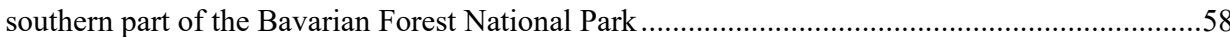

Figure 4.2 Cross validation of LWC estimation using LiDAR intensity .....................................65

Figure 4.3 An example of background pixels from hyperspectral image and canopy height model. (a) original canopy height model (b) dilated canopy height model; (c) and (d) spectral reflectance (red pixels indicate height below $0.1 \mathrm{~m}$ ), before and after dilation, respectively...........................66 Figure 4.4 Mean and spectral variability of the soil reflectance spectrum (dotted line: standard soil; solid lines: from remote sensing derived background)

Figure 4.5 Scatterplot showing the relationship between canopy cover obtained from hemispherical photos and the LiDAR index

Figure 4.6 Scatterplots between observed leaf water content and predicted leaf water content obtained from (a) inversion of LUT using hyperspectral only; (b) using prior information of remote sensing soil reflectance; (c) using all prior information

Figure 4.7 Leaf water content mapping of Bavarian Forest National Park using LiDAR and

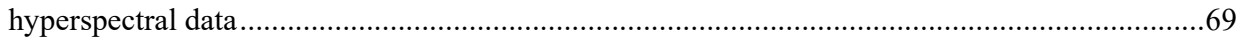

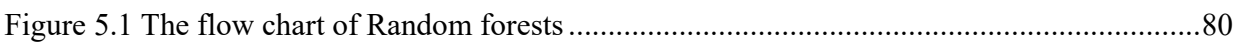

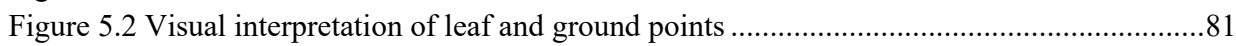

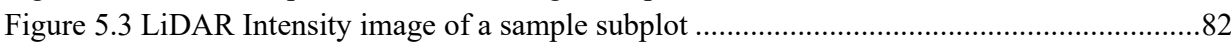

Figure 5.4 Distribution of LiDAR point clouds for different features; (a) frequency distribution of mean intensity data, (b) distribution of mean intensity and surface feature of point clouds ...........82 Figure 5.5 Visual comparison of classification results based on (a) radiometric features, (b) geometric features, and (c) the combination of both radiometric and geometric features ..............83 Figure 5.6 Visual comparison of classification results using based on different searching radius for two sample plots; (a) $\mathrm{r}=0.2 \mathrm{~m}$, (b) $\mathrm{r}=0.3 \mathrm{~m}$, (c) $\mathrm{r}=0.4 \mathrm{~m}$ and (d) adaptive radius......................85 Figure 5.7 Comparison of the ratio between foliage and woody materials derived from TLS and hemispherical photo for the 10 sample plots .....

Figure 5.8 TLS point cloud colorized by RGB photos and its final classification result for a mixed

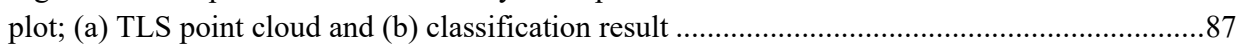
Figure 6.1 The backscatter intensity of two species with same leaf water content. (a) Before removal of the specular intensity and (b) after removal of the specular intensity.....................................93 Figure 6.2 3D Leaf water content mapping $\left(\mathrm{g} / \mathrm{cm}^{2}\right)$. (a) Polyscias fabian and (b) Camellia japonica.

Figure 6.3 Relationships between three waveform parameters (i.e., amplitude, backscatter crosssection, and backscatter coefficient) and LWC before correcting for incidence angle effect. .......95 Figure 6.4 Relationships between the backscatter coefficient and LWC after correcting for incidence angle effect (The circled points indicate extremely high LWC) .................................96 Figure 6.5 Scatterplot of the measured and estimated leaf water content by the backscatter coefficient. (a) excluding extreme case and (b) including extreme case.......................................96

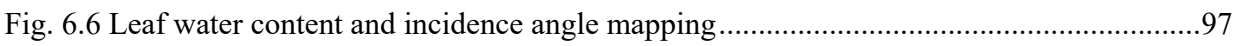

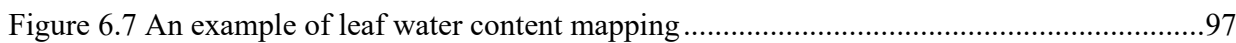
Figure 6.8 Results of FAST first-order sensitivity coefficients to canopy reflectance with modified INFORM (Cw: leaf water content, Cm: dry mater content, LAIs: single tree LAI, LAIu: understory LAI, CC: canopy cover, H: height, CD: crown diameter, Backref: background reflectance)........99 Figure 6.9 Canopy cover mapped using LiDAR data in the Bavarian Forest National Park, Germany 
Figure 6.11 The distribution of the ground, wood, and foliage in the geometric (surface feature: the shape of the local points is close to a surface) and radiometric domain.

Figure 6.12 Visual comparison of classification results based on (a) radiometric features, (b) geometric features, and (c) the combination of both radiometric and geometric features ............103 Figure 6.13 Classification results for a broadleaf plot and a needle leaf plot. (a) broadleaf, (b) needle leaf.

\section{List of tables}

Table 2.1 Statistics of the leaf water content of modeling samples

Table 2.2. Polynomial $R^{2}$ of the correlation between terrestrial laser scanner backscatter intensity at a perpendicular angle and leaf water content for eight plant species individually.....

Table 2.3 Terrestrial laser scanner backscatter intensity simulation for 8 plant species using linear combination of the Lambertian model and Beckmann law. $\mathrm{k}_{\mathrm{d}}$ : diffuse fraction, $\mathrm{m}$ : surface roughness

Table 3.1 Summary statistics of the measurements

Table 3.2 Mean $R^{2}$ and RMSE values of waveform decomposition comparison between Gaussian decomposition $(\mathrm{G})$ and skewed Gaussian decomposition (SG)

Table 4.1 Summary of statistics regarding in situ measurements of leaf and canopy variables collected in Bavarian Forest National Park. (LMA: leaf dry mass per area, CD: crown diameter, SH: stand height, CC: canopy cover).

Table 4.2 The input parameters and their ranges for LUT generation using INFORM ..................64

Table 4.3 Summary of statistics for leaf water content of different forest types.............................70

Table 5.1. Plot characteristics collected during the fieldwork in Bavarian Forest National Park, Germany.

Table 5.2 List of the radiometric and geometric features used/ extracted in this study from TLS data

Table 5.3 Feature importance values derived from Random Forests classifier ( : smaller than 0.02)

Table 5.4 Classification performance of using different features ................................................ 83

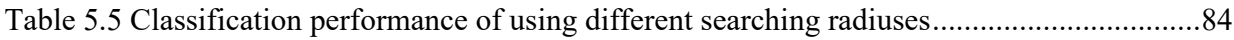

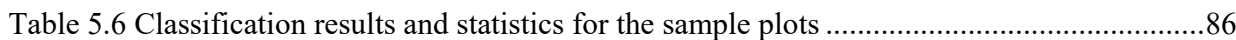

Table 5.7 Spearman's partial correlation test between the slope, understory coverage and the accuracy (bold: P value $<0.05$ )

Table 6.1 Terrestrial laser scanner backscatter intensity simulation for 2 plant species using a linear combination of the Lambertian model and Beckmann law. $\mathrm{k}_{\mathrm{s}}$ : specular fraction..... 93

Table 6.2 Summary of statistics for leaf water content of different forest types............................100

Table 6.3 Average accuracy of the classification results using different searching radius ...........104 
Chapter 1

Introduction 


\subsection{Ecological importance of leaf water content}

Forests provide a wide variety of goods and ecosystem services for humans. In order to improve the evaluation of forest conditions and changes, frequent and spatially continuous measurements of forest essential biodiversity variables are needed (Asner et al. 2016). Leaf water content (LWC) is one of the important essential biodiversity variables (Skidmore et al. 2015). It plays a key role in physiological processes and ecosystem functions such as photosynthesis, transpiration, thermal regulation, net primary production process and forest fire susceptibility and propagation (Chuvieco et al. 2004; Sanchez et al. 1983; Scriber 1977b). LWC may be used as an (early) indicator of plant and environmental stress (de Jong et al. 2012). As an essential biodiversity variable, LWC is important for ecosystem function and ecosystem structure evaluation (Skidmore et al. 2015). The qualification of LWC and knowledge on its spatial variation provide important information to assess future forest change associated with climate change, which can further help forest management and resource decision making (Asner et al. 2016).

\subsection{Role of remote sensing in the estimation of leaf water content}

Traditionally, LWC is estimated by conventional in situ destructive sampling, which is costly and time-consuming (Peñuelas et al. 1993) and is often restricted in a small spatial extent which limits its applicability (Tucker 1980). At a landscape scale, in situ observations are rarely sufficiently dense to accurately characterize its regional variation in LWC (Davidson et al. 2006). Especially in remote areas, in situ observations are often not feasible (Fleming 1988). Remote sensing techniques provide a non-destructive, rapid and economical way for the estimation of LWC across a wide range of spatial and temporal scales (Asner et al. 2016; Colombo et al. 2008; Yi et al. 2014).

In principle, the estimation of LWC using remote sensing is based on the relationship between spectral reflectance and LWC that relies on the spectral absorption features associated with LWC throughout the near-infrared (750-1300 nm) (Peñuelas et al. 1993), shortwave infrared (1300-2500 $\mathrm{nm}$ ) (Asner and Martin 2008), and mid-infrared to thermal infrared (3000-15000 nm) (Ullah et al. 2014) spectral regions. The changes in reflectance depending on LWC can be recognized and quantified as water content variations (Colombo et al. 2008).

Among a variety of different parameters, two are mainly used to describe plant water status by remote sensing, namely gravimetric water content (GWC, \%) and leaf equivalent water thickness (EWT, g/ $\mathrm{cm}^{2}$ ) (Yebra and Chuvieco 2009). The former refers to the proportion of leaf water relative to leaf dry mass which is more common in the fire risk literature (i.e., fuel moisture content) (Chuvieco et al. 2002), while the latter expresses leaf water content in mass per unit leaf area which is used in radiative transfer models 
(Ceccato et al. 2001). Studies show that reflectance is more related to changes in leaf EWT rather than changes in GWC (Ceccato et al. 2001; Colombo et al. 2008). Estimating EWT is usually more accurate than GWC. To estimate GWC, two independent variables (i.e., EWT and leaf dry mass area) both affecting leaf optical properties need to be estimated simultaneously (Yebra and Chuvieco 2009). Therefore, EWT is used throughout this work.

\subsubsection{LiDAR remote sensing}

Light detection and ranging (LiDAR) is a remote sensing method that uses visible and infrared light in the form of a pulsed laser to measure ranges (variable distances) to the earth (Us Department of Commerce and Atmospheric 2012). LiDAR systems produce a quantitative $3 \mathrm{D}$ digital representation of the objects in a given field of view with a measurement uncertainty (Vosselman and Maas 2010). LiDAR is an active remote sensing technique, which is not affected by solar illumination or shadowing (Woodhouse et al. 2011). Additionally, LiDAR can provide both horizontal and vertical information, enabling it to eliminate the influence of background, understory and canopy geometry (Morsdorf et al. 2006). The ability to capture 3D information makes LiDAR a powerful tool for forest structure characterization (Guang and Moskal 2012; Lefsky et al. 1999).

There are two types of LiDAR instruments - pulsed laser and continuous wave laser. The pulsed laser is most commonly used for forest applications (Lim et al. 2003). It measures the round-trip time of a short light pulse from the system to the target and back to the receiver (Mallet and Bretar 2009). Amongst various applications, two kinds of system can be identified: discrete echo system and full waveform system.

Discrete return LiDAR only provides the signal at a certain time of the echo, measuring the range $(\mathrm{R})$ to a target by recording the time delay $(\mathrm{t})$ created by a laser pulse from the source to the target (Vosselman and Maas 2010).

$$
R=c \cdot \frac{t}{2}
$$

where $\mathrm{c}$ is the speed of light.

Discrete return LiDAR systems identify major peaks in the return signal that represent discrete objects (Fig. 1.1). The potential of discrete return LiDAR for characterizing forest structure has long been demonstrated (Lovell et al. 2003; Morsdorf et al. 2006; Morsdorf et al. 2004). Depending on the application, LiDAR instruments can be used with a terrestrial laser scanner (TLS) or mounted on airborne (ALS) and spaceborne platforms. Most forest applications using LiDAR rely on ALS for large area data acquisition (Hilker et al. 2010). High-density ALS has a good chance to penetrate the vegetation canopy to provide ground information, so tree height can be correctly estimated. The accuracy relies largely on the individual tree detection and segmentation (Hyyppä et al. 2008). The variables obtained from segmentation can be later used for further applications such as 
biomass estimation and species classification (Ni et al. 2017; Zhao et al. 2009). The individual tree segmentation may result in large errors in dense forest where tree canopies are grouped (Zhao et al. 2009). Another method to derive forest structural variables is based on the discrete metrics within pre-defined grids or plots (Hilker et al. 2010; Solberg et al. 2009). This method requires an empirical model between the in situ measurement and LiDAR metrics, which can be used to estimate various structural variables such as canopy cover and leaf area index (LAI) (Korhonen et al. 2011; Tang et al. 2014a). Airborne discrete return LiDAR is able to cover large areas more efficiently, but it fails to detect the lower canopy and the details of different vegetation elements due to the relatively large footprint size and low sampling density (Hilker et al. 2012; Vierling et al. 2013). The advent of TLS filled the gap between costly traditional field measurements and relatively low-resolution airborne data, providing a variety of canopy characterizations at a fine scale. Due to its $3 \mathrm{D}$ nature and small footprints, it is able to capture the information of single leaves without being affected by the existence of background and other vegetation components (Zhu et al. 2018). In addition, it can be positioned under the canopy to reduce the shadowing effects and the obstruction of overstory canopy (Vierling et al. 2013). A growing number of studies have used terrestrial LiDAR to quantify forest parameters including both biophysical and biochemical parameters such as LAI, leaf angle and chlorophyll content (Eitel et al. 2010b; Jupp et al. 2009; Loudermilk et al. 2009; Loudermilk et al. 2007; Moorthy et al. 2008; Strahler et al. 2008; Vierling et al. 2013).

In addition to geometric information, discrete return LiDAR can also provide the backscatter intensity of each return. The definition of intensity varies in different studies, while in this study, the intensity is defined as the peak amplitude of the returned waveform. The backscatter intensity recorded by LiDAR instruments is a function of the reflectance property of an object (Penasa et al. 2014). As an active remote sensing technique, the intensity is, to some extent, insensitive to ambient light and atmospheric conditions. It provides good spectral separability for objects identification and classification (Höfle and Pfeifer 2007). Despite the challenges of calibration for angle and distance effects, the intensity has been used in combination with geometric information for various forest applications including species classification, gap fraction modeling and biomass estimation (García et al. 2010; Kim et al. 2009; Solberg et al. 2008). However, little is known about how useful backscatter intensity data are for LWC estimation.

Full-waveform LiDAR systems record the continuous signal from the reflected energy, allowing advanced processing of the echo's full shape which increases pulse detection reliability, accuracy and resolution (Mallet and Bretar 2009; Vosselman and Maas 2010) (Fig. 1.1). Full-waveform systems also provide additional information about the structure and physical backscattering properties of the targets such as the backscatter coefficient (Mallet and Bretar 2009; Wagner 2010). These backscatter properties derived from fullwaveform data are very useful for classification as well as vegetation structure estimation 
(Lindberg et al. 2012; Yao et al. 2012). Fieber et al. (2013) derived reflectance values of orange trees from the backscatter coefficient of single-peak waveforms, which were consistent with published reflectance values. It hints that waveform data have the potential for biochemical variables estimation.
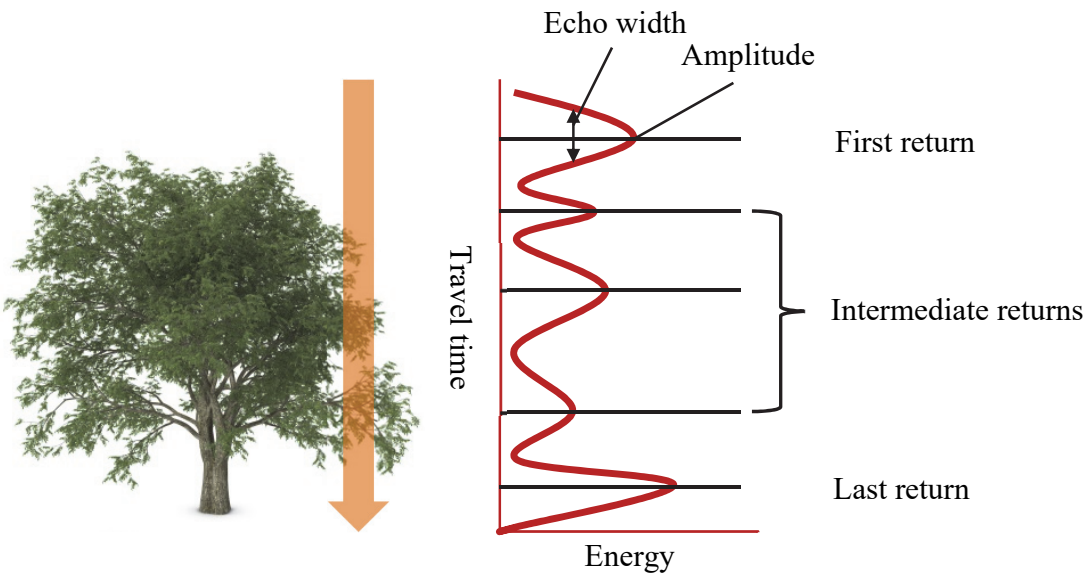

Figure 1.1 Interaction of laser pulse with vegetation and waveform decomposition

\subsubsection{Hyperspectral remote sensing}

Broad band satellite data have been used for the assessment for LWC using either empirical models or physical models (Ceccato et al. 2002; Jackson et al. 2004; ZarcoTejada et al. 2003). However, the spectral characteristics of vegetation biochemicals are oftern masked by convolving the incoming radiation across broad wavelength ranges (Broge and Leblanc 2001). By contract, hyperspectral data can provide detailed narrow spectral information (Houborg et al. 2009; Ma et al. 2014). Hyperspectral sensors can acquire contiguous spectrum for each image pixel over a selected wavelength interval (Goetz 2009). The strength of hyperspectral remote sensing in the observation of vegetation is found in the exactness of the spectral response due to its contiguous, narrow spectral channels (Anderson et al. 2008). In the late 1980's, many hyperspectral imagers became commercially available in the market (Goetz 2009). Since then, hyperspectral remote sensing has been used to retrieve various essential biodiversity variables with good accuracy (Asner et al. 2015; Meroni et al. 2004; Wang et al. 2017). Gong et al. (2003) constructed 12 vegetation indices from 168 Hyperion bands for forest LAI estimation with a high accuracy. Darvishzadeh et al. (2008b) successfully estimated canopy chlorophyll content with a canopy reflectance model in a grassland using hyperspectral measurement. Skidmore et al. (2010) used hyperspectral remote sensing to map the simultaneous distribution of foliar nitrogen and polyphenol in African mopane savannas and explained how the variation of forage quality can influence management. The contiguous sampling characteristic has rendered hyperspectral remote sensing an 
effective technique especially for LWC estimation, as it is capable of detecting the absorption features of leaf water with its narrow bands. Gao and Goetzt (1995) showed a good relationship between LWC and hyperspectral reflectance data, obtaining a good agreement with an $\mathrm{R}$ value of 0.78 . Champagne et al. (2003) applied a physical model using hyperspectral data to directly calculate LWC with an index of agreement (D) of 0.92. Cheng et al. (2006) used a canopy reflectance model to retrieve LWC, showing a consistent relationship between retrieved and measured LWC.

Amongst these studies, the methods for LWC estimation can be categorized mainly into two groups: empirical and physical approaches. Estimation using empirical models based on the relationship between ground-based LWC and reflectance or its transformation (Houborg et al. 2007). The estimation of LWC using empirical approaches has been demonstrated in numerous studies (Cheng et al. 2011; Seelig et al. 2008; Ullah et al. 2013). They provide a good accuracy at the local scale and can be applied without high computational demands (Richter et al. 2007). However, the main drawback of empirical approaches is their lack of generality (Curran and Williamson 1986). When transferred across vegetation types and study sites, reliable in-situ measurements are required for model calibration (Darvishzadeh et al. 2008b).

On the other hand, physical approaches take into account physical processes describing the interaction between solar radiation and vegetation components based on radiation transfer models (Jacquemoud 1993). Radiative transfer models (RTM) offer an explicit physical connection between vegetation biophysical and biochemical variables and reflectance, thus offer advantages in transferability and robustness compared to statistical approaches (Darvishzadeh et al. 2012; Schlerf and Atzberger 2006). RTMs inversion allows the estimation of various vegetation variables, while the inversion can be ill-posed since different combinations of input parameters may produce the same spectral signature (Yebra and Chuvieco 2009). At leaf level, leaf RTMs (e.g. PROSPECT, LIBERTY) have been established to model the interaction between leaf components and radiation (Dawson et al. 1998; Jacquemoud and Baret 1990). They have been successfully used for LWC estimation at leaf level (Bowyer and Danson 2004; Ceccato et al. 2001; Yi et al. 2014).

Canopy reflectance models accurately describe canopy reflectance, as a function of canopy, leaf and background characteristics (Atzberger 2000). Four main categories of canopy reflectance models can be distinguished (Schlerf and Atzberger 2006): Turbid medium models (1-D radiative transfer model; e.g. Suits (1971); Verhoef (1984)) characterized forest canopy layer as horizontally homogeneous and infinitely extended, which is unsuited for the sparse forest that is horizontally heterogeneous. Darvishzadeh et al. (2011) used PROSAIL to map grassland LAI with airborne hyperspectral data with accuracies comparable to those of statistical approaches. Geometric models (e.g. Li and Strahler (1986)) assume that the canopy consists of a series of regular geometric shapes, placed on the ground surface in a prescribed manner (Liang 2005). Consequently, crown 
transparency is assumed to be zero. The neglected transmissivity of tree crowns is a fundamental weakness of these models (Atzberger 2000). Ray-tracing models (e.g. North (1996); Kobayashi and Iwabuchi (2008)) can accurately compute the radiation distribution over a complex canopy configuration. However, due to the complex structure and a large number of input parameters required, these models are computationally expensive and difficult to invert (Schlerf and Atzberger 2006). Hybrid models (e.g. Huemmrich (2001); Li et al. (1995)) are combinations of geometric and turbid medium models. These types of models provide a compromise between the realism of simulation of canopy and invertibility (Schlerf and Atzberger 2006). Yang et al. (2011) presented a new forest LAI inversion method from multisource and multiangle data using a hybrid model of the invertible forest reflectance model (INFORM) with an $\mathrm{R}^{2}$ value of 0.772 (Atzberger 2000). (Ali et al. 2016b) investigated the relationship between leaf dry matter content and specific leaf area with the canopy reflectance using INFORM model, which demonstrated the advantage of INFORM for canopy reflectance simulation and plant traits estimation.

\subsubsection{Integration of LiDAR and hyperspectral}

Hyperspectral remote sensing has the advantage for estimating leaf biochemical variables at many levels, while it also has certain limitations. At the regional scale, canopy structure often confounds the link between leaf variables and canopy reflectance (Niemann et al. 2012; Wang et al. 2017). In addition, background reflectance is another source that weakens the relationship between leaf variables and the reflectance. Both empirical and physical approaches are challenged with these issues. Water-related optical indices are not only related to LWC but also LAI and canopy cover (Zarco-Tejada et al. 2003). The large variation of canopy structural variables makes the parameterization of physical models challenging, resulting in the so-called "ill-posed problem" in the inversion procedure (Yebra and Chuvieco 2009). Many radiative transfer models have incorporated background reflectance as a separate layer (Atzberger 2000; Verhoef and Bach 2003), but from hyperspectral data alone, it is very difficult to extract the background layer in the mixed pixels. These limitations of hyperspectral remote sensing can be partly overcome by the integration of LiDAR. The advantage of LiDAR lies in its capability to capture 3D structural information, which offers a complement for hyperspectral remote sensing. However, most currently LiDAR systems employ a single wavelength. The relationship between the desired varible and the intensity of a single wavelength is often weakened by enviromental, instrumental and geometric factors (Höfle and Pfeifer 2007). The integration of hyperspectral and LiDAR remote sensing has the potential to overcome the weekness of both hyperspectral and LiDAR data.

\subsection{Challenges in leaf water content estimation}

The estimation of LWC faces many challenges at different scales. Terrestrial LiDAR has very high point density coupled with a small footprint, rendering it an effective tool for 
LWC estimation at the leaf scale. However, TLS backscatter intensity for LWC estimation has not been explored, since the intensity is affected by the distance and incidence angle effects. The distance effect is mainly dominated by instrumental factors, so the calibration is straightforward (Kaasalainen et al. 2011). Nonetheless, the incidence angle effect is largely dependent on the target surface properties (Krooks et al. 2013). The intensity decreases with increasing incidence angle due to the energy dispersion, while the rate of decrease is difficult to evaluate before knowing the surface properties of the target (Zhu et al. 2015). A study by Krooks et al. (2013) showed that the incidence angle effect is dominated by target reflectance. This finding offered a useful perspective for intensity calibration.

At the individual canopy scale, except for the influence of distance and incidence angle, estimating LWC using TLS intensity is also complicated by the effect of partial hits at the leaf edge (Gaulton et al. 2013). This effect is not significant at the leaf scale, but at the canopy scale, a large number of laser beams partially hit the edge of the leaves thereby reducing the fraction of returned energy (Eitel et al. 2010b). This partial hits effect has to be eliminated before the intensity can be used for LWC estimation. Current studies show that dual-wavelength TLS such as SALCA (Salford Advanced Laser Canopy Analyser) has the ability to remove the influence of partial hits by using an intensity ratio of two bands (Danson et al. 2014). However, the challenge of partial hits calibration remains for single wavelength TLS which is still the most commonly used terrestrial LiDAR instrument.

At the regional scale, there are also a few challenges when estimating LWC using airborne data. As airborne LiDAR has a relatively big footprint (much larger than single leaves), it is not feasible to calculate the incidence angle between each leaf and the laser beam. In addition, when the laser beam hits multiple targets, it is not directly related to the target reflectance, since the intensity is not only a function of the target reflectance, but also the portion of the beam hitting the target (Béland et al. 2011). Unlike TLS, due to the large footprint size of ALS, most laser beams hit multiple targets within the footprint. With insufficient prior knowledge about full hits from the same target, the true reflectance of partially hit individual targets cannot be unmixed because the target reflectance and the collision area between the laser beam and partial hits both contribute to the returned intensity (Béland et al. 2014). On the other hand, canopy reflectance models using hyperspectral data simulating the spectral bidirectional reflectance as a function of many important forest characteristics are well established (Atzberger 2000). The downside of hyperspectral data is as mentioned: the inability to fully eliminate forest structure and background effects. Therefore, in order to accurately estimate LWC at the regional scale, the use of an individual sensor is not sufficient. A number of studies have revealed that more accurate results can be achieved by integrating both sensor types (Fu et al. 2011; Thomas et al. 2007; Torabzadeh et al. 2014). However, the integration of LiDAR and 
hyperspectral data in radiative transfer modeling has not been attempted for LWC estimation.

An additional important factor that contributes to the bias of the estimation of leaf variables (e.g. LAI, LWC) is the presence of plant woody material, especially at the canopy scale. Hosoi and Omasa (2007) pointed out that one of the most significant factors that affected the estimation accuracy of LAI was the presence of woody material. When the remote sensing signal is a mixture of foliar and woody materials, the estimated variable is a plant variable, instead of a pure leaf variable. Thus, the accurate estimation of leaf variables requires an accurate classification of foliar and woody materials. On a 2D image, the complete unmixing between these two classes is not possible since not only are they horizontally mixed, but they are also vertically mixed. Additionally, the footprint size and point density of airborne LiDAR are not fine enough for the separation between foliar and woody materials. In comparison, TLS is able to capture the 3D spatial arrangement of different vegetation components due to its small footprint size and high point density (Côté et al. 2009). The geometric information provided by TLS has been assessed for the classification in a few studies (Ma et al. 2014; Zheng et al. 2016a), but the combination of TLS radiometric (i.e. intensity) and geometric information has received little attention.

\subsection{Research questions}

The aim of this study is to retrieve LWC at the leaf and canopy level, as well as explore upscaling to the regional level, using LiDAR and hyperspectral remote sensing. Four research questions are outlined below:

(1) Does the calibration of terrestrial LiDAR intensity data to a common leaf angle significantly increase the estimation accuracy of LWC?

(2) Can canopy LWC be estimated using terrestrial LiDAR by removing the influence of partial hits?

(3) To what extent does the integration of LiDAR and hyperspectral improve the accuracy of LWC estimation when coupled in RTMs?

(4) How is the performance of the combination of geometric and radiometric features for the classification of foliar and woody materials compared to the usage of either one of them alone?

\subsection{Thesis structure}

This thesis comprises six chapters, four of which are standalone papers addressing the research questions presented in section 1.4. Three of them have been published in peerreview ISI journals, one is under review. 
Chapter 2 evaluates the ability of terrestrial LiDAR intensity to estimate LWC with radiometric calibration at the individual leaf scale. Chapter 3 assesses the feasibility of estimating canopy LWC using terrestrial LiDAR by removing the influence of partial hits. Chapter 4 focuses on LWC estimation at the regional scale by using LiDAR and hyperspectral data. Chapter 5 presents a method to classify foliar and woody materials through the combination of radiometric and geometric information using terrestrial LiDAR. Finally, the thesis is concluded with a synthesis of the research presented in Chapter 6 . The research findings are discussed, and future research and suggestions are proposed.

\subsection{Study area}

The experiments of Chapter 2 and Chapter 3 were conducted in the Geoscience Laboratory in the Faculty of Geo-Information Science and Earth Observation of the University of Twente, Enschede, the Netherlands. The study area of Chapter 4 and Chapter 5 was located in the Bavarian Forest National Park in southeastern Germany (Fig. 1.2). The natural forest ecosystems of the Bavarian Forest National Park vary according to altitude: there are alluvial spruce forests in the valleys, mixed mountain forests on the hillsides and mountain spruce forests in the high areas (Heurich et al. 2010).

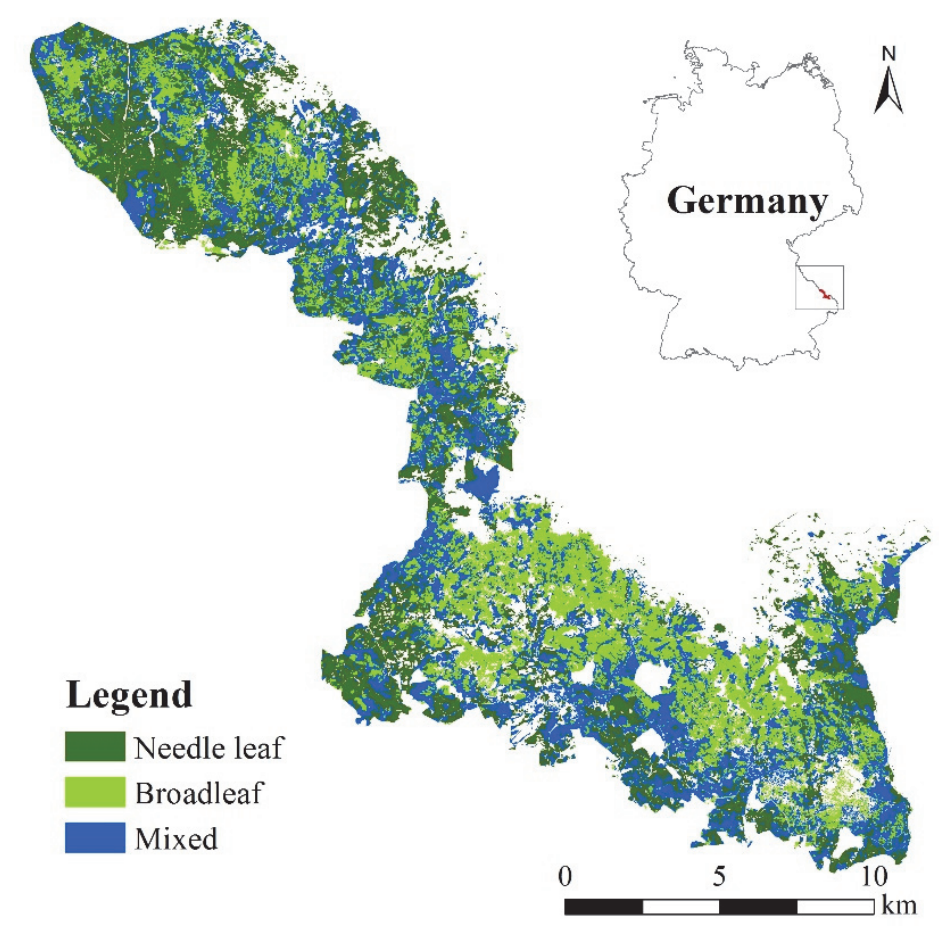

Figure 1.2 Location of the study area in Germany 


\subsection{Data description}

The terrestrial laser scanner used in this study was the time-of-flight scanner RIEGL VZ400 (Riegl LMS GmbH, Horn, Austria) which has been widely applied in forestry studies. It employs a SWIR (1550 nm) laser, known to be sensitive to LWC (Tucker 1980). The system has a beam divergence of $0.35 \mathrm{mrad}$, a range accuracy of $5 \mathrm{~mm}$, an effective measurement rate of 122,000 meas./sec, and a maximum range of $160 \mathrm{~m}$ at $20 \%$ reflectance in a high speed mode. The pulse's energy follows a Gaussian distribution within the laser beam.

The airborne LiDAR flight campaign was carried out at the end of July 2012 using a Riegl LMS-Q $680 \mathrm{i}$ sensor at a nominal height of $650 \mathrm{~m}$ above ground covering the whole park. The wavelength of the LiDAR data was $1550 \mathrm{~nm}$ and the data had an average point density of 30 points per $\mathrm{m}^{2}$. The system had a beam divergence smaller than $0.5 \mathrm{mrad}$, a range accuracy of $20 \mathrm{~mm}$ and a maximum pulse repetition rate of 400,000 $\mathrm{Hz}$.

Airborne hyperspectral data were obtained with a HySpex sensor by German Aerospace Center (DLR) for the study area on July 22, 2013. The system comprises two imaging spectrometers with spectral ranges from 400 to $1000 \mathrm{~nm}$ across 160 channels (visible and near infrared, VNIR) and from 1000 to $2500 \mathrm{~nm}$ across 256 channels (short-wave infrared, SWIR). The spatial resolutions of these two spectrometers were $1.65 \mathrm{~m}$ and $3.3 \mathrm{~m}$, respectively. The data were collected at an average nominal altitude of $3000 \mathrm{~m}$ in 19 image strips. The data were preprocessed by DLR. Image data were converted from digital numbers to surface reflectance using the atmospheric correction model ATCOR4 based on atmospheric lookup tables generated with the radiative transfer model MODTRAN4 (Wang et al. 2016). Ortho-rectification was performed based on the parametric model/table using recorded attitude and flight path data in combination with a digital terrain model (DEM) (Wang et al. 2017). 


\section{Chapter 2 \\ 3D leaf water content mapping using \\ terrestrial laser scanner backscatter intensity with radiometric correction*}

* This chapter is based on: Zhu, X., Wang, T., Darvishzadeh, R., Skidmore, A.K., \& Niemann, K.O. (2015). 3D leaf water content mapping using terrestrial laser scanner backscatter intensity with radiometric correction. ISPRS Journal of Photogrammetry and Remote Sensing, 110, 14-23. 


\begin{abstract}
Leaf water content (LWC) plays an important role in agriculture and forestry management. It can be used to assess drought conditions and wildfire susceptibility. Terrestrial laser scanner (TLS) data have been widely used in forested environments for retrieving geometrically-based biophysical parameters. Recent studies have also shown the potential of using radiometric information (backscatter intensity) for estimating LWC. However, the usefulness of backscatter intensity data has been limited by leaf surface characteristics, and incidence angle effects. To explore the idea of using LiDAR intensity data to assess LWC we normalized (for both angular effects and leaf surface properties) shortwave infrared TLS data $(1550 \mathrm{~nm})$. A reflectance model describing both diffuse and specular reflectance was applied to remove strong specular backscatter intensity at a perpendicular angle. Leaves with different surface properties were collected from eight broadleaf plant species for modeling the relationship between LWC and backscatter intensity. Reference reflectors (Spectralon from Labsphere, Inc.) were used to build a look-up table to compensate for incidence angle effects. Results showed that before removing the specular influences, there was no significant correlation $\left(R^{2}=0.01, P>\right.$ 0.05 ) between the backscatter intensity at a perpendicular angle and LWC. After the removal of the specular influences, a significant correlation emerged $\left(R^{2}=0.74, P<0.05\right)$. The agreement between measured and TLS-derived LWC demonstrated a significant reduction of RMSE (root mean square error, from 0.008 to $0.003 \mathrm{~g} / \mathrm{cm}^{2}$ ) after correcting for the incidence angle effect. We show that it is possible to use TLS to estimate LWC for selected broadleaved plants with an $R^{2}$ of 0.76 (significance level $\alpha=0.05$ ) at leaf level. Further investigations of leaf surface and internal structure will likely result in improvements of 3D LWC mapping for studying physiology and ecology in vegetation.
\end{abstract}




\subsection{Introduction}

Vegetation water content provides useful information for assessing plant physiological status (Sanchez et al. 1983; Scriber 1977a) and is critical in parameterization of radiative transfer models (Dawson et al. 1998; Jacquemoud and Baret 1990). In addition, it is commonly used to assess forest fire susceptibility and propagation (Chuvieco et al. 2002; Ray et al. 2005) and many forest fire behavior models rely on it as an input (Bowyer and Danson 2004; Cruz et al. 2004; Rothermel et al. 1986). Remote sensing techniques provide an efficient and non-destructive way to estimate vegetation water content. Passive multi-spectral and hyper-spectral data have been widely used for retrieving vegetation water content from 2-dimensional (2D) images using either empirical models (Gao 1996; Serrano et al. 2000; Ullah et al. 2014) or physical models (Jacquemoud and Baret 1990; Kötz et al. 2004; Zarco-Tejada et al. 2003). Tucker (1980) simulated spectral reflectance changes in the 700-2500 $\mathrm{nm}$ region using different leaf water contents (LWC), suggesting that the $1480-1750 \mathrm{~nm}$ spectral interval was the best-suited region for ground-based monitoring of vegetation water status. Serrano et al. (2000) showed that reflectance indices formulated from near infrared (NIR) water absorption bands were the best indicators of vegetation water content. Ullah et al. (2014) investigated the spectral region 390-14000 $\mathrm{nm}$ for retrieving LWC using narrow-band indices. The results showed that the most accurate spectral index could yield very high prediction accuracy $\left(R^{2}=0.93\right)$. However, such passive remote sensing could be affected by solar illumination and atmospheric conditions. In addition, 2D imaging techniques do not differentiate between different vegetation elements (e.g. leaves, branches), nor do they eliminate background soil and understory vegetation signal (Eitel et al. 2010a; Wagner et al. 2008a; Wessman 1994). Chuvieco et al. (2002) stated that the main challenge of using passive remote sensing is that reflectance is affected by factors of spectral variation, such as canopy geometry, soil background or atmospheric effects that confound the foliar biochemical signal.

Terrestrial laser scanners (TLS) have the potential to overcome many intrinsic limitations of optical platforms. Their data are independent of solar illumination, and are thus able to obtain the measurements at optimal viewing angles where no shadowing is present (Woodhouse et al. 2011). In addition, the 3-dimensional (3D) measurement techniques allow the separation of background noise such as understory vegetation and background soil using vertical information (Höfle 2014) or TLS backscatter intensity (Pirotti et al. 2013). TLS data have been extensively used for obtaining 3D geometrical information using more traditional airborne and spaceborne Light Detection and Ranging (LiDAR) modeling techniques. The high point-density of TLS enables vegetation information to be explored at small spatial scales (mm) (Vierling et al. 2013). The backscatter intensity value of the reflected backscatter signal recorded by TLS is a function of the reflectance property of an object (Penasa et al. 2014), which is, to some extent, insensitive to ambient light and atmospheric conditions (Höfle and Pfeifer 2007) and provides good spectral 
separability for detecting and classifying objects (Franceschi et al. 2009; Höfle 2014). Although little is known about how useful backscatter intensity values are for retrieving leaf biochemical characteristics, such as estimating LWC, some recent studies support the use of TLS in this realm. Eitel et al. (2010a) utilized the green band TLS (532 nm) to measure leaf chlorophyll content for two tree species, and showed that the green band backscatter intensity is strongly correlated with leaf chlorophyll content $\left(R^{2}=0.77\right)$. In another study, green band TLS backscatter intensity data were shown to be useful for estimating the foliar nitrogen concentration of spring wheat (Eitel et al. 2011b). Magney et al. (2014) found a strong relationship between green laser return intensity and nonphotochemical quenching, demonstrating that green laser return intensity holds promise to provide detailed information about plant physiological status. Gaulton et al. (2013) used a dual-wavelength laser scanning for LWC estimation. A strong relationship $\left(R^{2}=\right.$ $0.80)$ was found between a normalized ratio of the two wavelengths (1063 nm and 1545 $\mathrm{nm})$ and LWC. Other studies have examined the ability of TLS with SWIR wavelength for classifying minerals due to the strong water absorption in this region (Franceschi et al. 2009; Penasa et al. 2014). Béland et al. (2011) separated the photosynthetic part from the non-photosynthetic of six trees in order to obtain leaf area index using TLS backscatter intensity at the wavelength of $1535 \mathrm{~nm}$, since the water absorption in this band is higher in leaves than in branches (Tucker 1980). These studies show that TLS with a specific wavelength can be used to obtain certain leaf biochemical parameters and their exact spatial distribution. Therefore, it is feasible to use TLS with water absorption band to estimate LWC. With its 3D geometric and radiometric information, TLS has the potential to provide information on 3D plant structure and biochemical content for each measurement point at different parts of a plant. It can therefore be useful for analyzing plant physiology.

Using TLS backscatter intensity to estimate LWC is complicated by the leaf angle distribution, because the incidence angle between the laser beam and the leaf normal affects TLS backscatter intensity strongly (Eitel et al. 2010a; Gaulton et al. 2013). Gaulton et al. (2013) showed that a normalized ratio of two wavelengths from a dualwavelength laser is highly correlated with vegetation moisture content. The normalized ratio of two wavelengths should be insensitive to the incidence angle, since the effect is partially cancelled when the backscatter intensity of both wavelengths is similarly influenced by the incidence angle (Eitel et al. 2014; Gaulton et al. 2013). These newly emerged instruments provide means for removing incidence angle effects, while for the widely used TLS instruments with single wavelength, correction for incidence angle effects is necessary. Even for the dual-wavelength laser scanning, the effect can be removed by using the ratio only when the two wavelengths are similarly affected by the incidence angle (Eitel et al. 2014). Thus, radiometric correction for incidence angle effects is still necessary. The incidence angle effect mainly depends on the target's properties, including reflectivity and roughness (Kaasalainen et al. 2011). TLS backscatter intensity decreases as incidence angle increases due to the energy dispersion 
caused by spot spreading, while the rate of angle-dependent decay increases with the backscatter intensity measured at normal incidence if a regular surface is considered (Pesci and Teza 2008). Kukko et al. (2008) presented a comprehensive experiment how backscatter intensity depends on the incidence angle using a set of natural and artificial samples. They demonstrated that the incidence angle effect is stronger for high reflectance targets. The angle effect also depends on the surface roughness of targets with a large grain size compared to the laser spot size, while reflectance other than roughness plays a stronger role for targets with small grain size (Kukko et al. 2008). Krooks et al. (2013) investigated the role of surface properties such as grain size and reflectance on the angledependent decay of backscatter intensity. They showed that the incidence angle effect is dominated by target reflectance, whereas the variations in surface topography that are smaller than the laser footprint on the target do not have a significant influence on the incidence angle effect. This suggests that the incidence angle effect can be corrected using functions that depend on the object brightness (Krooks et al. 2013). Leaf surface topography is usually smaller than the laser spot size which is about $7 \mathrm{~mm}$ at a distance of $2 \mathrm{~m}$ for the instrument used in this study. Therefore, radiometric correction of TLS should allow object brightness to be accurately normalized to reflectance.

There are three generally accepted approaches for radiometric correction of LiDAR backscatter intensity: The first is a theoretical model based on the radar equation describing the relationship between the transmitted signal power and received signal power (Ding et al. 2013). In a perfect Lambertian distribution, the backscatter intensity value is related to the cosine of the incidence angle (Coren and Sterzai 2006). The main limitation of this approach is that most objects do not follow the Lambertian scattering law. Besides, some TLS systems do not follow the radar equation at close ranges due to optical vignetting or brightness reducers (Pfennigbauer and Ullrich 2010). A second approach employs an empirical model using predefined homogeneous samples (Höfle and Pfeifer 2007) to estimate parameters for a function accounting for angle-dependent factors. This method is very laborious and precludes automation of the backscatter intensity correction process (Ding et al. 2013). The third method uses a reference targetbased model based on commercial or natural reference targets (Kaasalainen et al. 2009) to acquire the different rate of angle-dependent decay caused by surface brightness. The reference target-based model can avoid laborious measurements, as it uses the same model based on standard reference targets for various species.

Before correcting for the angle effect, the relationship between LWC and backscatter intensity at an angle perpendicular to the TLS-object nadir needs to be established. However, a challenge to attain this relationship is to remove the specular backscatter intensity component for this wavelength $(1550 \mathrm{~nm}$ ) (Eitel et al. 2014). Although the contribution of specular backscatter intensity from the leaf surface may have little effect on total backscatter intensity from a different incidence angle, it has a noticeable effect at a perpendicular angle (Sinclair et al. 1973). At a perpendicular angle, there is a large 
fraction of specular backscatter intensity that varies due to the different surface features (Ding et al. 2013). As discussed by Eitel et al. (2010a), shiny leaf surfaces have a higher specular fraction of backscatter intensity than matte leaves and exhibit different backscatter intensity values than matte leaf surfaces, especially at a perpendicular angle ,even though they have the same leaf chlorophyll content. Thus, for LWC estimation, the backscatter intensity at a perpendicular angle may be affected by leaf surface type, which also needs to be corrected. Here we hypothesize that a reflectance model which incorporates both specular and diffuse backscatter intensity can eliminate surface feature effects across species, so that the correlation between backscatter intensity and LWC can be improved.

The overall objective of this study was to examine the suitability of TLS backscatter intensity data to estimate LWC. Specifically, we aimed: (1) to examine the correlation between LWC and TLS backscatter intensity at a perpendicular angle; (2) to investigate the influence of the leaf surface feature on backscatter intensity and to assess the application of a reflectance model on leaf backscatter intensity; and (3) to evaluate the incidence angle effect on backscatter intensity variation and LWC mapping.

\subsection{Materials and methods}

\subsubsection{Terrestrial laser scanner}

The laser scanner used in our study was the time-of-flight scanner RIEGL VZ-400 (Riegl LMS GmbH, Horn, Austria; Fig. 2. 1) which has been widely applied in forestry studies. It employs a SWIR (1550 nm) laser, known to be sensitive to LWC (Tucker 1980). The system has a beam divergence of $0.35 \mathrm{mrad}$, a range accuracy of $5 \mathrm{~mm}$, an effective measurement rate of 122,000 meas./sec, and a maximum range of $160 \mathrm{~m}$ at $20 \%$ reflectance in a high speed mode. The pulse's energy follows a Gaussian distribution within the laser beam. All of the data were acquired in a high speed mode with a pulse repetition rate (PRR) of $300 \mathrm{kHz}$.

Discrete return systems record the return amplitude of each received echo as backscatter intensity, while a large number of LiDAR systems are able to record full-waveform information providing range, peak amplitude (backscatter intensity) and the shape of the waveform (Jupp et al. 2005). RIEGL VZ-400 can provide both full waveform output and discrete returns. The result of online waveform processing is a stream of data providing precise information on target range, amplitude and reflectance for each detected target echo (RIEGL Laser Measurement Systems, 2014). The reflectance provided is a ratio of the actual amplitude of that target to the amplitude of a white flat target at the same range. It has been used as the backscatter intensity in this study for estimating LWC. The main advantage of this output is that the range-dependent effect has been removed (Pfennigbauer and Ullrich 2010). 


\subsubsection{Experiment design and TLS measurement}

Leaves (13-18 samples per species, 127 in total) were randomly collected from eight broadleaf plant species for modeling the relationship between LWC and TLS backscatter intensity. The eight plant species were Piggyback Plant (Tolmiea menziesii), Smooth Hydrangea (Hydrangea arborescens), Rhododendron (Rhododendron sp.), Garden Croton (Codiaeum variegatum), Red Robin (Photinia fraseri), Dwarf Umbrella Tree (Schefflera arboricola), Ficus Tree (Ficus benjamina) and Zanzibar Gem (Zamioculcas zamiifolia) (Fig. 2.2 from left to right; Table 2.1). As can be seen in Fig. 2.2, Zamioculcas zamiifolia and Ficus benjamina have shinier leaves than other species, while Tolmiea menziesii and Hydrangea arborescens have hairy and matte leaves. Other species have visually moderate shiny leaves. Four additional broadleaf plant species were used for validation on plants without detaching: Kangaroo Vine (Cissus antartica), Aralia Fabian (Polyscias fabian), African Hemp (Sparrmannia africana) and Cherry Laurel (Prunus laurocerasus).

To study the incidence angle effect on backscatter intensity, four Spectralon panels manufactured by Labsphere, Inc. with nominal reflectance values of $12 \%, 25 \%, 50 \%$, $99 \%$ were scanned at incidence angles between $0^{\circ}$ and $80^{\circ}$, in steps of $10^{\circ}$ (Fig. 2.1). A simple goniometric platform was built to measure the leaf backscatter intensity change with angle (Fig. 2.2). It can be rotated horizontally to change the incidence angle, whereas the elevation was fixed to the same height as the laser scanner. A protractor was fixed on the platform to show the incidence angle. The platform board was painted matte black to differentiate leaves from the background. Leaves were detached from the eight plant species, placed in an oven to dry progressively and taken out every hour for measuring backscatter intensity and weight. They were flattened and attached to the board for the backscatter intensity measurements made at a perpendicular angle to obtain the correlation between water content and backscatter intensity. In total, we obtained 463 measurements with different levels of water content for modeling (Table 2.1). Backscatter intensity was also measured at incidence angles of $0^{\circ}$ to $30^{\circ}$ in steps of $2^{\circ}$ to simulate the specular and diffuse fractions. The one sided area of each leaf was computed by multiplying the number of leaf pixels by the pixel area. Leaf pixels were distinguished on the scanned image by performing a segmentation with the ENVI software (ITT Visual Information Solutions Inc., USA).

The LWC was calculated using the following formula (Danson et al. 1992):

$$
(M W-M D) / A\left(\mathrm{~g} / \mathrm{cm}^{2}\right)
$$

where $M W$ is the mass of the wet leaf $(\mathrm{g}), M D$ is the mass of the completely dried leaf (g), and $A$ is the surface area of the leaf $\left(\mathrm{cm}^{2}\right)$. 


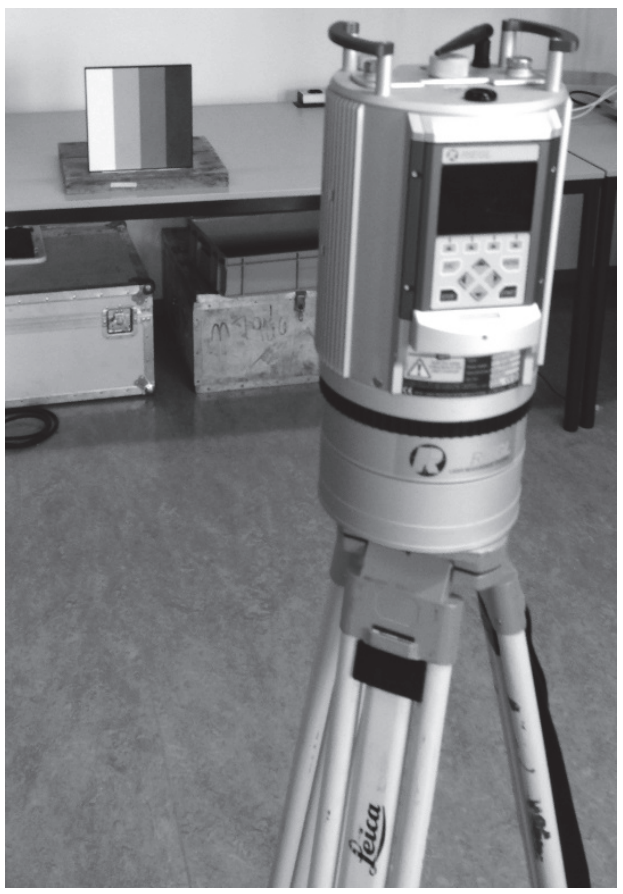

Figure 2.1 The terrestrial laser scanner measuring Spectralon panels

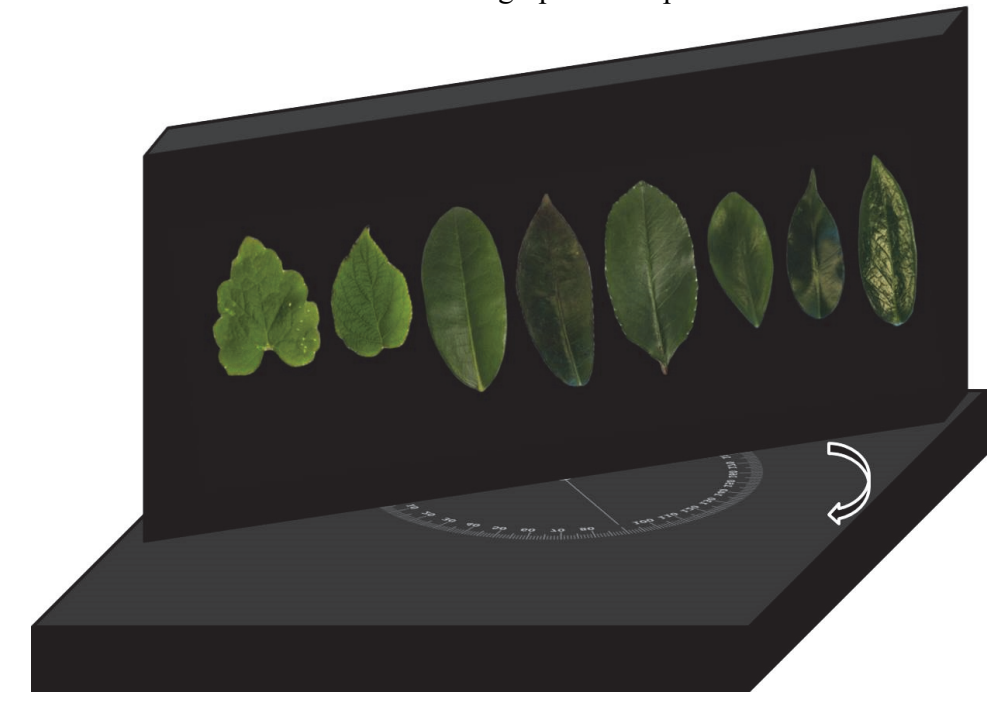

Figure 2.2 Leaves of 8 plant species attached to a goniometric platform 
Table 2.1 Statistics of the leaf water content of modeling samples

\begin{tabular}{|c|c|c|c|c|c|}
\hline Species name & Measurements & $\begin{array}{l}\text { Max LWC } \\
\left(\mathrm{g} / \mathrm{cm}^{2}\right)\end{array}$ & $\begin{array}{l}\text { Min LWC } \\
\left(\mathrm{g} / \mathrm{cm}^{2}\right)\end{array}$ & $\begin{array}{l}\text { Mean } \\
\text { LWC } \\
\left(\mathrm{g} / \mathrm{cm}^{2}\right)\end{array}$ & $\begin{array}{l}\text { Standard } \\
\text { deviation of } \\
\mathrm{LWC}\left(\mathrm{g} / \mathrm{cm}^{2}\right)\end{array}$ \\
\hline $\begin{array}{l}\text { Tolmiea } \\
\text { menziesii }\end{array}$ & 64 & 0.0133 & 0.0047 & 0.0089 & 0.0021 \\
\hline $\begin{array}{l}\text { Hydrangea } \\
\text { arborescens }\end{array}$ & 48 & 0.0109 & 0.0024 & 0.0070 & 0.0019 \\
\hline $\begin{array}{l}\text { Rhodedendron } \\
s p\end{array}$ & 96 & 0.0236 & 0.0064 & 0.0147 & 0.0034 \\
\hline $\begin{array}{l}\text { Codiaeum } \\
\text { variegatum }\end{array}$ & 39 & 0.0195 & 0.0062 & 0.0137 & 0.0038 \\
\hline Photinia fraseri & 80 & 0.0194 & 0.0036 & 0.0114 & 0.0044 \\
\hline $\begin{array}{l}\text { Schefflera } \\
\text { arboricola }\end{array}$ & 58 & 0.0370 & 0.0080 & 0.0240 & 0.0080 \\
\hline Ficus benjamina & 36 & 0.0192 & 0.0030 & 0.0113 & 0.0049 \\
\hline $\begin{array}{l}\text { Zamioculcas } \\
\text { zamiifolia }\end{array}$ & 42 & 0.0536 & 0.0273 & 0.0457 & 0.0064 \\
\hline In total & 463 & 0.0536 & 0.0024 & 0.0161 & 0.0114 \\
\hline
\end{tabular}

\subsubsection{Reflectance model for terrestrial laser scanner backscatter intensity simulation}

Natural surface reflectance of an object is a combination of diffuse and specular reflectance. Thus, a mixed Lambertian/non-Lambertian model is needed for both the diffuse and specular reflectance modeling. The specular reflectance is the mirror-light reflectance of light from a surface. A linear combination of the Lambertian model and Beckmann law (Poullain et al. 2012), which provides a comprehensive theory that can be applied to a wide range of surface conditions ranging from smooth to very rough, was used to simulate the backscatter intensity. The Lambertian model defines the diffuse intensity for the dull, matte surfaces, while the Beckmann law (Beckmann and Spizzichino 1987) models specular intensity properties.

$$
I=f\left(k_{d} \cos \alpha+\left(\left(1-k_{d}\right) \exp \left(-\left(\tan ^{2} \alpha / m^{2}\right)\right) /\left(\cos ^{5} \alpha\right)\right)\right)
$$

where $I$ is the backscatter intensity, $f$ represents backscatter intensity at normal incidence angle, $k_{d}$ is the fraction of the diffuse intensity, $\alpha$ is the incidence angle, and $m$ is the surface roughness and typically takes values between 0 (smooth surface) and 0.6 (rough surface). 


\subsubsection{Incidence angle effect correction on backscatter intensity}

\subsubsection{Incidence angle}

The incidence angle is the angle between the surface normal and the laser beam. Thus, the surface normal is required. The K-nearest neighbor (KNN) algorithm was used to search for the nearest neighbors of each point, since KNN can cope with boundaries, holes and different sampling densities (Hoppe et al. 1992). Then the surface normal was estimated based on the analysis of the eigenvectors and eigenvalues of a covariance matrix from the nearest neighbors of the query point (Gross and Thoennessen 2006). The incidence angle was therefore calculated from the following equation:

$$
\alpha=\arccos (\mathbf{P} \cdot \mathbf{N} /|\mathbf{P N}|)
$$

where $\alpha$ is the incidence angle, and $\mathbf{P}$ is the laser beam vector from the laser scanner to the surface. $\mathbf{N}$ is the surface normal vector.

\subsubsection{Models for incidence angle correction}

In this paper, the reference target-based model is used to test its potential for retrieving LWC, while the Lambertian model is used as a baseline to compare the results.

\section{- $\quad$ Theoretical model for incidence angle correction}

According to the radar range equation (Jelalian 1992), the backscatter intensity observed from an ideal diffusely reflecting surface or ideal diffuse radiator is directly proportional to the cosine of the angle $\alpha$. Assuming that footprints on objects are circular, the surface has Lambertian scattering characteristics and that atmospheric conditions are constant, it can be simplified to the following equation (Höfle and Pfeifer 2007):

$$
I_{r}=I_{t} \rho \eta_{a t m} \cos \alpha \cdot C / R^{2}
$$

where $I_{r}$ is the received signal power, $I_{t}$ is the transmitting signal power, $\rho$ is the target reflectance, $R$ is the distance from sensor to target, $\eta_{a t m}$ is the atmospheric attenuation constant, $\alpha$ is the incidence angle, and $C$ is the sensor system constant factor.

Since $I_{t}, \eta_{\text {atm }}$, and $C$ were constant, $R$ has been corrected (Pfennigbauer and Ullrich 2010). $I_{r}$ is proportional to $\cos \alpha$. Therefore, for all points, the measured backscatter intensity was normalized by $\mathrm{I}=\mathrm{I}_{\mathrm{r}} / \cos \alpha$ considering the incidence angle (Jutzi and Gross 2009).

- Reference target-based model for incidence angle correction 
In order to correct the backscatter intensity, the observed backscatter intensity at different incidence angles for the four panels with different backscatter intensity at perpendicular angles $\left(\mathrm{I}\left(0^{\circ}\right)\right)$ was stored in a look-up table (LUT). The LUT is interpolated to provide I $\left(0^{\circ}\right)$ for any specific incidence angle and observed backscatter intensity, so that $\mathrm{I}\left(0^{\circ}\right)$ can be linearly interpolated from the model for a given backscatter intensity recorded by the instrument and incidence angle calculated (see 2.2.4.1). Fig. 2.3 gives a surface visualization of the radiometric calibration model.

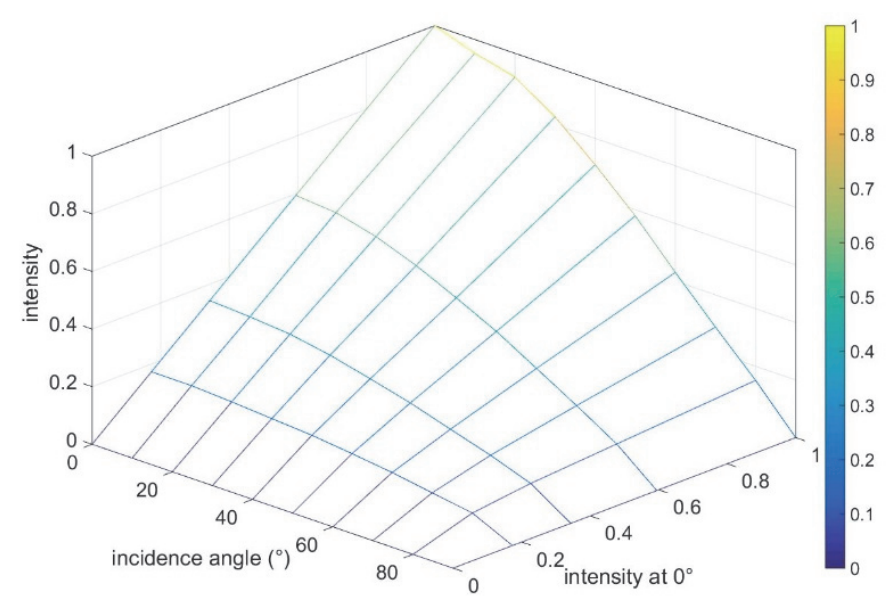

Figure 2.3 Surface visualization of the radiometric calibration model generated from observations with four Spectralon panels at multiple angles

\subsection{Results}

\subsubsection{Correlation between backscatter intensity and leaf water content at a perpendicular angle before removing specular backscatter intensity}

The relationship between TLS backscatter intensity and LWC is demonstrated in Fig. 2.4. It shows that for most species, TLS backscatter intensity at a perpendicular angle decreased with increasing LWC (Fig. 2.4a). This suggests that backscatter intensity is negatively correlated with LWC, since the energy of laser is partly absorbed by the leaves' water. When the LWC is higher, the absorptance of backscatter intensity at $1550 \mathrm{~nm}$ is stronger. Table 2.2 shows the polynomial correlation between TLS backscatter intensity and LWC for eight plant species. TLS backscatter intensity was correlated with LWC for each of the seven species, while there was no significant correlation observed for Zamioculcas zamiifolia $\left(R^{2}=0.01\right)$. Combining the eight plant species together, no significant correlation was found between TLS backscatter intensity and LWC (Fig. 2.4a). 

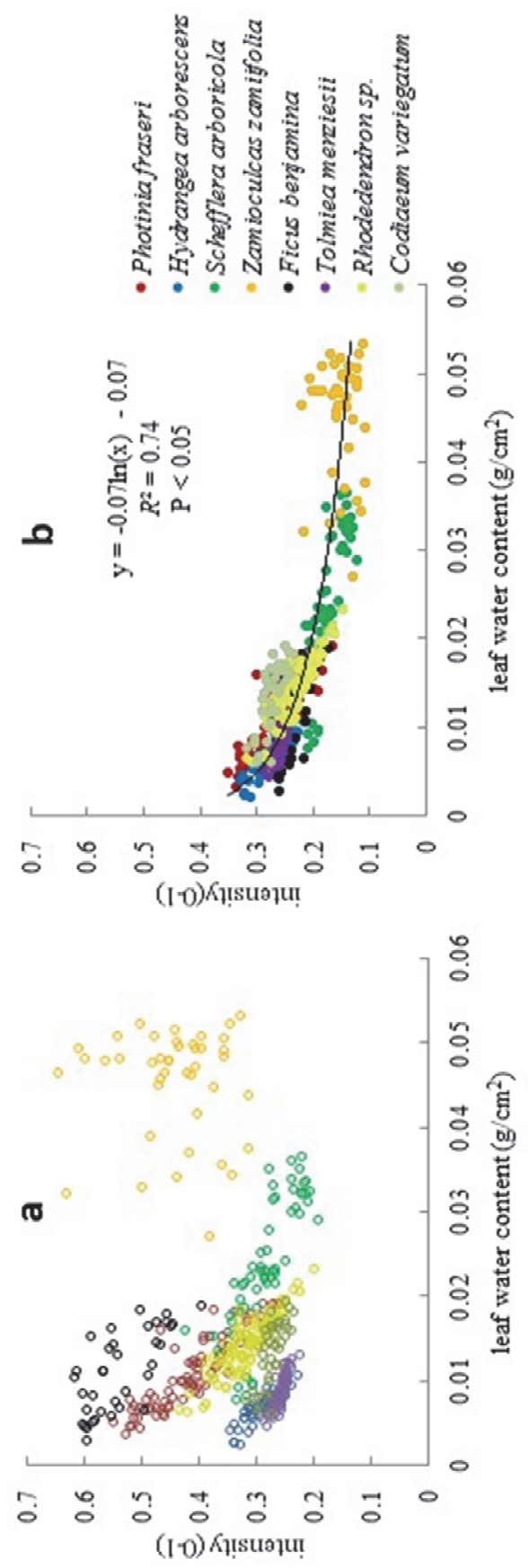

Figure 2.4 Relationships between leaf water content and backscatter intensity at a perpendicular angle. (a) before removal of specular backscatter intensity, (b) after removal of specular backscatter intensity. 
Table 2.2. Polynomial $R^{2}$ of the correlation between terrestrial laser scanner backscatter intensity at a perpendicular angle and leaf water content for eight plant species individually

\begin{tabular}{lll}
\hline Species name & $R^{2}$ & RMSE \\
\hline Zamioculcas zamiifolia & 0.01 & 0.09 \\
Ficus benjamina & 0.54 & 0.04 \\
Schefflera arboricola & 0.65 & 0.03 \\
Photinia fraseri & 0.80 & 0.03 \\
Codiaeum variegatum & 0.54 & 0.02 \\
Rhodedendron sp. & 0.78 & 0.02 \\
Hydrangea arborescens & 0.77 & 0.01 \\
Tolmiea menziesii & 0.75 & 0.01 \\
\hline
\end{tabular}

\subsubsection{Effect of removing the specular backscatter intensity on the correlation between leaf water content and backscatter intensity at a perpendicular angle}

\subsubsection{Terrestrial laser scanner backscatter intensity simulation using a linear combination of the Lambertian model and Beckmann law}

TLS backscatter intensity has been simulated to exclude the specular component in order to attain a more general model for LWC prediction using a linear combination of the Lambertian model and Beckmann law (Table 2.3). Zamioculcas zamiifolia and Ficus benjamina showed the lowest diffuse fractions and highest specular fractions compared to the other species. Fig. 4a shows that these two species exhibited higher specular backscatter intensity, while Hydrangea arborescens and Tolmiea menziesii had the lowest specular fractions. Table 2.3 also shows that leaves with higher ' $k_{d}$ ' values also had higher ' $\mathrm{m}$ ' values, suggesting that rough leaves might have higher diffuse fraction.

For species with a lower value of parameter $k_{d}$ (Table 2.3), with the same backscatter intensity at a perpendicular angle, the backscatter intensity dropped more significantly as the incidence angle increased (Fig. 2.5). For instance, Zamioculcas zamiifolia had the highest specular fraction (smallest $k_{d}$ value). At off-normal angles, it was the diffuse fraction that contributed most to the backscatter intensity, so the whole backscatter intensity dropped faster than for other species. On the other hand, Tolmiea menziesii had the lowest specular fraction, which was very close to a Lambertian surface (Fig. 2.5). 
Table 2.3 Terrestrial laser scanner backscatter intensity simulation for 8 plant species using linear combination of the Lambertian model and Beckmann law. $\mathrm{k}_{\mathrm{d}}$ : diffuse fraction, $\mathrm{m}$ : surface roughness

\begin{tabular}{lllll}
\hline Species name & $\mathrm{k}_{\mathrm{d}}$ & $\mathrm{m}$ & $R^{2}$ & RMES \\
\hline Zamioculcas zamiifolia & 0.34 & 0.09 & 0.95 & 0.03 \\
Ficus benjamina & 0.44 & 0.13 & 0.98 & 0.01 \\
Schefflera arboricola & 0.63 & 0.18 & 0.98 & 0.01 \\
Photinia fraseri & 0.64 & 0.21 & 0.94 & 0.02 \\
Codiaeum variegatum & 0.68 & 0.17 & 0.97 & 0.01 \\
Rhodedendron sp. & 0.73 & 0.20 & 0.99 & 0.01 \\
Hydrangea arborescens & 0.95 & 0.22 & 0.99 & 0.01 \\
Tolmiea menziesii & 0.99 & 0.38 & 0.83 & 0.01 \\
\hline
\end{tabular}

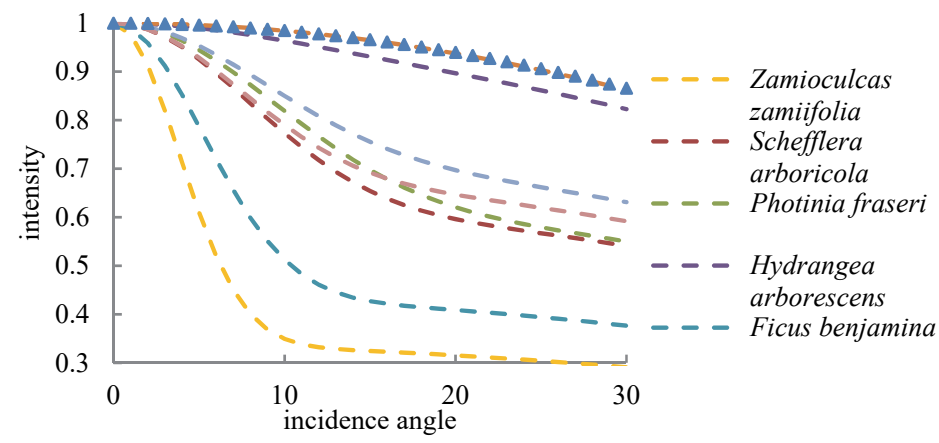

Figure 2.5 Linear combination of the Lambertian model and Beckmann law simulation for eight plant species. Backscatter intensity at a normal incidence angle has been scaled to 1 .

\subsubsection{Correlation between terrestrial laser scanner backscatter intensity and leaf water content at a perpendicular angle after removing the specular backscatter intensity}

TLS backscatter intensity at a perpendicular angle was corrected by subtracting the specular component. As expected, species with a higher backscatter intensity at the same LWC level, such as Zamioculcas zamiifolia and Ficus benjamina, had a higher specular backscatter intensity, although after correction, values dropped to the same level as other species (Fig. 2.4b), whereas the backscatter intensity of species which were less shiny experienced a smaller decrease after correction due to their lower specular fraction. Therefore, after removing specular backscatter intensity, when we pooled the samples from the eight plant species together (Fig. 2.4b), we obtained a more accurate $R^{2}$ value of 0.74 (compared to the uncorrected data shown in Fig. 2.4a). As expected, TLS backscatter intensity decreased as LWC increased, while it became less sensitive when LWC was high (Fig. 2.4b) (they had a logarithmic relationship). 


\subsubsection{Correction of incidence angle effect on terrestrial laser scanner backscatter intensity and leaf water content prediction}

Fig. 2.6 shows the recorded backscatter intensity as a function of incidence angle for four panels: when the reflectance was higher, the angle decay was more significant. This could also be observed for leaves (Fig. 2.7). Fig. 2.8 shows the result of estimating LWC using uncorrected TLS backscatter intensity: the LWC was significantly overestimated.

Fig. 2.9 shows the visualization of TLS backscatter intensity correction on a plant (Polyscias fabian). For leaves with a large incidence angle (off-nadir to TLS), the backscatter intensity was much lower than those with small incidence angle (Fig. 2.9a). After backscatter intensity correction, it was observed that most parts of the plant had a higher backscatter intensity. Especially for leaves with a large incidence angle, the backscatter intensity became much higher compared to leaves with a small incidence angle, suggesting the correction was effective. In addition, backscatter intensity of branches was significantly higher than that of the leaves.

The estimation of LWC using corrected TLS backscatter intensity data showed the improvement of $R^{2}$ and smaller RMSE (root mean square error) (Fig. 2.10). The reference target-based model provided a more accurate estimation of LWC, yielding an $R^{2}$ value of 0.76 and RMSE of $0.003 \mathrm{~g} / \mathrm{cm}^{2}$ compared to the theoretical model $\left(R^{2}=0.55\right.$, RMSE $=$ $\left.0.004 \mathrm{~g} / \mathrm{cm}^{2}\right)$. When LWC was above $0.02 \mathrm{~g} / \mathrm{cm}^{2}$, the LWC was overestimated, indicating the saturation problem of TLS backscatter intensity data for high LWC levels.

Fig. 2.11 gives a visualization of LWC spatial distribution on two plant species (Polyscias fabian and Camellia japonica). It provides the information of the LWC for every TLS point on the leaves, thus it is possible to determine the water status of leaves on the plant. It is clear that the venation had a higher water content than the other parts of a leaf (Fig. 2.11a). Fig. 2.11b shows more variation of LWC on a plant: most of the newer leaves close to the ends of the branches had a higher LWC than older leaves closer to the root.

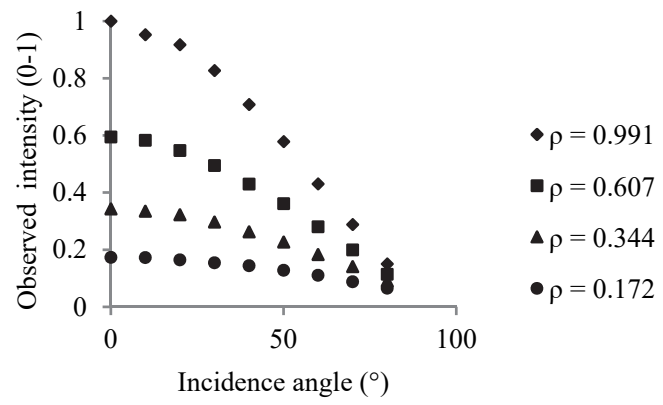

Figure 2.6 Terrestrial laser scanner backscatter intensity as a function of the incidence angle and reflectance for the Spectralon panels 


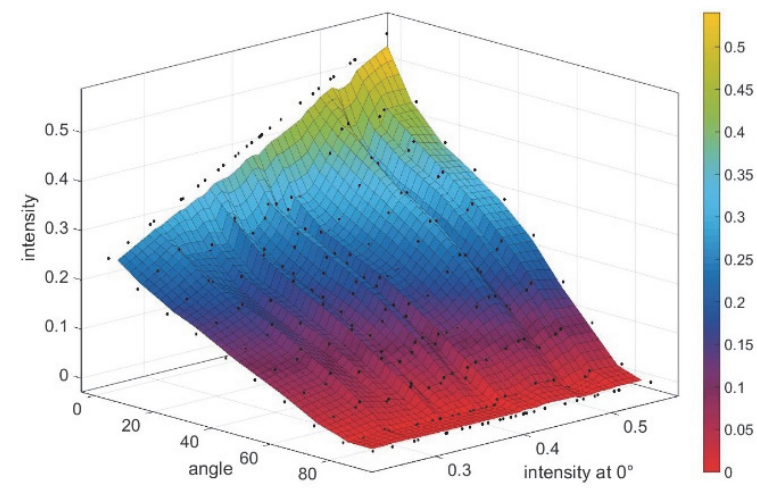

Figure 2.7 Leaf backscatter intensity changes with incidence angle and backscatter intensity at a perpendicular angle

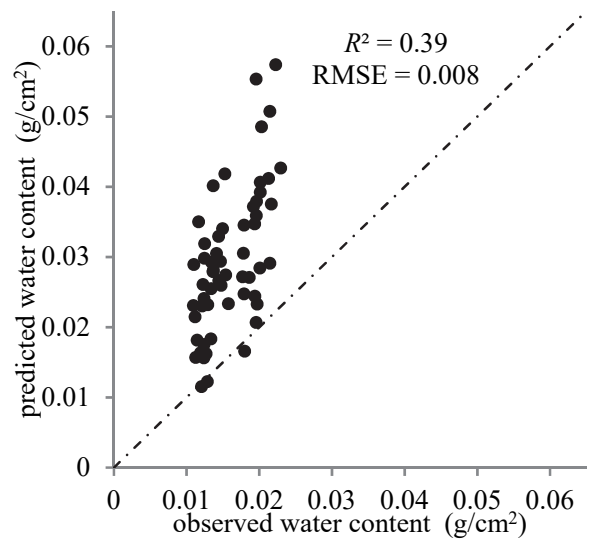

Figure 2.8 Leaf water content estimation using terrestrial laser scanner backscatter intensity before angle correction
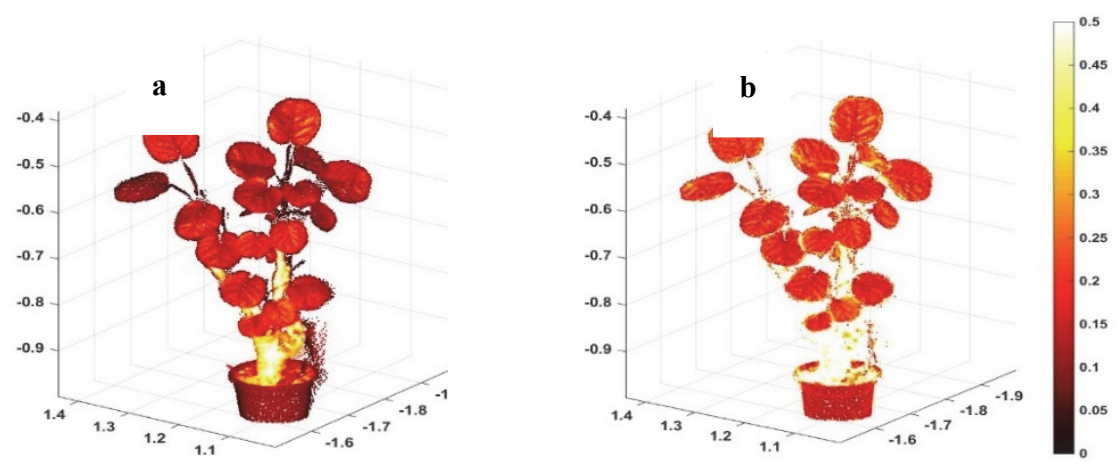

Figure 2.9 Terrestrial laser scanner backscatter intensity correction visualization. (a) before correction, (b) after correction 

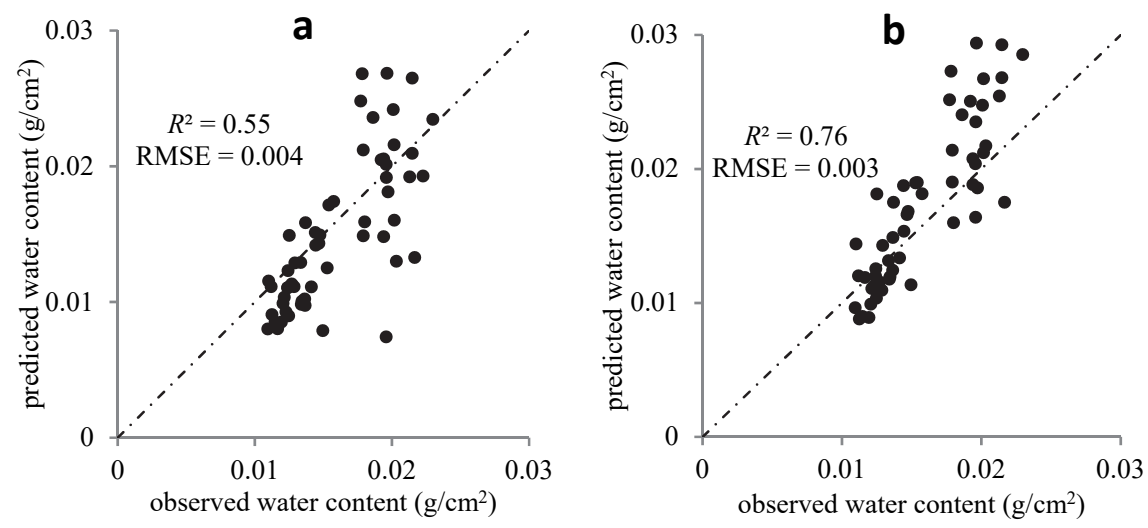

Figure 2.10 Leaf water content estimation using terrestrial laser scanner backscatter intensity after angle correction. (a) theoretical model based correction, (b) reference target based correction.
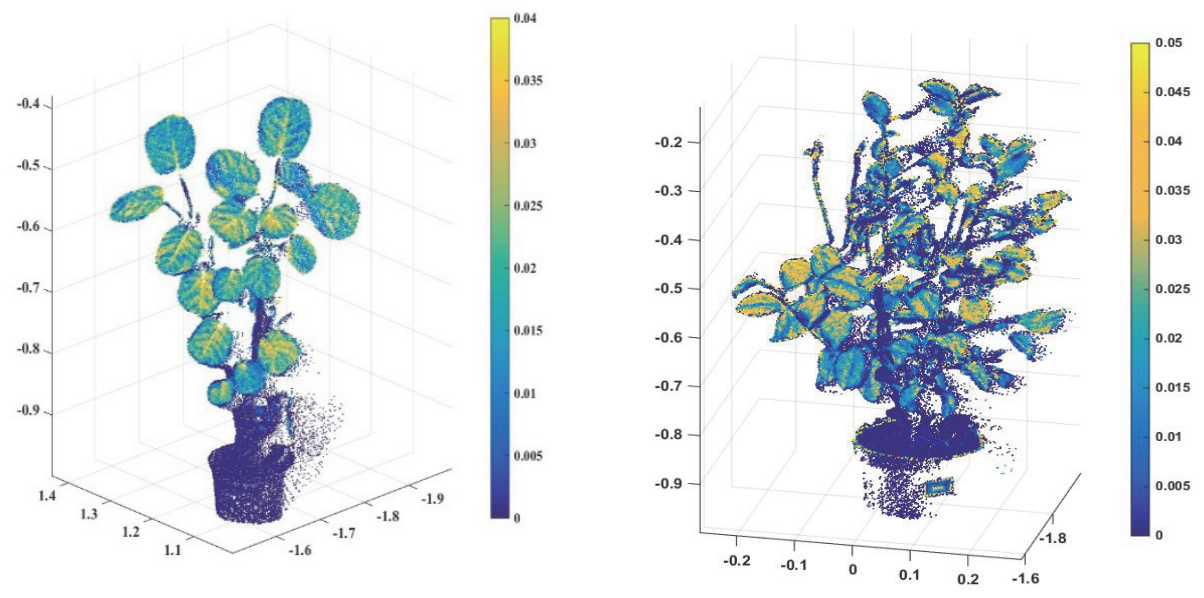

Figure 2.11 Example of leaf water content mapping. (a) Polyscias fabian, (b) Camellia japonica.

\subsection{Discussion and conclusion}

LWC estimation has so far mainly focused on hyperspectral passive remote sensing, which provides very high accuracy with an $R^{2}$ above 0.8 (Ullah et al. 2014; Zhang et al. 2012), however the advent of TLS allows the assessment of LWC and its exact spatial distribution. In this paper we have demonstrated that TLS with a SWIR wavelength can be used to estimate LWC for selected broadleaved plants at an acceptable accuracy $\left(R^{2}=\right.$ $\left.0.76, \mathrm{RMSE}=0.003 \mathrm{~g} / \mathrm{cm}^{2}, \alpha=0.05\right)$ at leaf level.

Before removal of the specular component, LWC was correlated with TLS backscatter intensity at a perpendicular angle for most species, although in the case of Zamioculcas 
zamiifolia, there was no significant correlation $(P>0.05$; Fig. $2.4 \mathrm{a})$. There are three possible reasons for this. Firstly, the LWC of Zamioculcas zamiifolia is very high (0.03$\left.0.06 \mathrm{~g} / \mathrm{cm}^{2}\right)$, so if the LWC is too high, it appears to induce the laser signal saturates. Fig. $2.4 \mathrm{~b}$ demonstrates that above a certain LWC level (around 0.02-0.03 $\mathrm{g} / \mathrm{cm}^{2}$ ), TLS backscatter intensity becomes less sensitive to LWC (logarithmic function). This can also be observed from Fig. 2.10, both the methods show that above about $0.02 \mathrm{~g} / \mathrm{cm}^{2}$, the prediction accuracy tends to be lower. This saturation phenomena of LWC estimation has been discussed in other studies using hyperspectral SWIR data (Ceccato et al. 2001) and thermal infrared data (Ullah et al. 2012). More research is needed to fully understand and solve this issue. Secondly, the diffuse fraction of Zamioculcas zamiifolia is very low $\left(\mathrm{k}_{\mathrm{d}}\right.$ $=0.34$ ) so that the dominant backscatter intensity at a perpendicular angle is 'specular'. Specular backscatter intensity varies widely at low incidence angles. Therefore, the backscatter intensity before removal of the specular component is affected by small changes of incidence angle, which explains the variation of TLS backscatter intensity at the same LWC level. Thirdly, when dried in the oven, the leaf surface of Zamioculcas zamiifolia became less shiny leading to the decrease of its specular fraction. This change is also present in other species, to various extents. When the leaf surface is shinier, the influence tends to be stronger.

After pooling the eight plant species together, before correcting for specular backscatter intensity, the TLS backscatter intensity showed no significant correlation with LWC (Fig. 2.4a). Leaf reflectance emanates from both the interior of the leaf and the leaf surface. The diffusive character of leaf backscatter intensity is derived from the multiple scattering of radiation within the leaf, whereas the specularly reflected intensity is a function of leaf surface characteristics (Grant 1987). At a perpendicular angle, the specular backscatter intensity is more noticeable (Sinclair et al. 1973). Different plant species may have the same LWC, but with different surface features. Shiny leaf surfaces exhibit a higher TLS backscatter intensity than matte leaf surfaces, as they are more affected by specular backscatter intensity at a perpendicular angle. This is especially true of Zamioculcas zamiifolia and Ficus benjamina, whose backscatter intensity values are much higher than other species at the same LWC level (Fig. 2.4a). A linear combination of the Lambertian model and Beckmann law successfully quantifies the difference of diffuse and specular fraction (Table 2.3). In Fig. 2.5, the decreasing backscatter intensity of Tolmiea menziesii was very close to a Lambertian surface due to its low specular backscatter intensity. After removing the specular backscatter intensity, a good correlation was found $\left(R^{2}=0.74\right)$ for the eight plant species pooled together, suggesting that the simulation and correction are effective (Fig. 2.4b). LWC difference can be clearly reflected by TLS backscatter intensity, since diffuse backscatter intensity is less affected by leaf surface.

Correcting the incidence angle effect on TLS backscatter intensity improved the accuracy of LWC retrieval (Fig. 2.10). We used reference targets of different brightness levels to model angle-dependent backscatter intensity decay. In Fig. 2.7 we show that the 
brightness difference (backscatter intensity $\left(0^{\circ}\right)$ ) has a notable influence on the leaf backscatter intensity decay with incidence angle, suggesting that brightness needs to be considered as a factor for backscatter intensity correction. Brightness dominates the incidence angle effect on TLS backscatter intensity, rather than grain size, due to the smaller leaf size (grain) compared with the laser footprint size. We will investigate grain size effect and its correction in future studies.

The reference target-based model is superior in correcting for backscatter intensity compared to the theoretical model (Fig. 2.10). The theoretical model assumes that leaves are perfect Lambertian reflectors that follow the cosine incidence angle law. In reality, leaves are not perfect Lambertian surfaces and the relationship between received optical power and reported backscatter intensity DNs (digital number) is nonlinear (Hartzell et al. 2015). However, radiometric calibration methods based on the theoretical model (radar equation) using normalized or raw backscatter intensity assume a linear relationship between TLS backscatter intensity and received optical power. For reference target-based models, the backscatter intensity at a perpendicular angle may be interpolated from the LUT without assuming a linear relationship.

In this study, the relative reflectance output of the online full waveform processing was used for LWC estimation. The online full waveform analysis converts the full waveform into multiple returns with range, amplitude, relative reflectance and pulse shape deviation. But in our controlled experiment, there were only single returns in modelling (leaves attached on the board) and very few multiple returns in validation, since the laser beam size was very small and the density of vegetation used in validation was low. Thus, the multiple returns of the online waveform output have little impact on the estimation of LWC. However, in a dense forest, the multiple returns could have larger influence on the result, because for multiple returns, only a fraction of the laser beam can reach the target. The backscatter intensity is therefore attenuated, resulting in inaccurate estimation of LWC. Using single return data might be a better choice in this condition, as it is very difficult to assess the area of collision and the received power on the target for multiple returns.

Riegl VZ-400 can also output the full waveform data in addition to the discrete returns. The raw waveform contains the whole energy reflected by a target, taking into consideration the duration of the received signal, instead of only amplitude (Heinzel and Koch 2011; Wagner et al. 2008b). In addition, more parameters can be retrieved from the waveform, such as echo width, backscatter cross section and coefficient using Gaussian decomposition. Echo width has shown to be related to surface roughness (Lin and Mills 2010), which may benefit LWC estimation across species. The backscatter cross section and coefficient have been applied on classification using airborne data in many studies (Alexander et al. 2010; Mallet et al. 2011). Since the backscatter cross section and coefficient are directly related to the biconical reflectance (Wagner 2010), they might be 
more correlated to LWC than backscatter intensity. However, these studies mainly focused on airborne data, the decomposition and calibration of TLS waveform data are not direct due to brightness saturation or logarithmic amplifiers (Hartzell et al. 2015). Further research is needed to address these issues and compare the result from offline full waveform analysis.

The visualization of backscatter intensity and LWC in Fig. 2.9 and Fig. 2.11 demonstrates the differences of LWC in the 3D plant structure. The backscatter intensity of branches is much higher than that of leaves (Fig. 2.9), suggesting that the water content of the branches is lower than that of green leaves because the absorptance is higher in leaves than in branches (Béland et al. 2011). Several studies have used LWC to classify plant photosynthetic and non-photosynthetic tissue, especially for leaf area index estimation (Béland et al. 2011; García et al. 2011). In addition, there are differences of water content inside a leaf: the water content of the venation is higher than other parts. The principal function of the venation of a leaf is to deliver water (Roth-Nebelsick et al. 2001) so it is logical that the LWC is higher. However, we would note that the TLS used in this study has only one band, viz. $1550 \mathrm{~nm}$, which is not only affected by LWC, but also by the leaf's internal structure (Ceccato et al. 2001), so this will require further investigation, for example by correcting for the venation leaf structure component of the signal. Fig. 2.11b also shows that the LWC of older leaves is lower than for new and emerging leaves. Mooney et al. (1977) demonstrated that when leaves are fully hydrated, new leaves have a higher water content than older leaves. 3D LWC mapping has the potential to provide more useful information for physiological and ecological studies in vegetation, such as drought and fuel loading assessment. 


\section{Chapter 3 \\ Canopy leaf water content estimated using terrestrial LiDAR*}

* This chapter is based on: Zhu, X., Wang, T., Skidmore, A.K., Darvishzadeh, R., Niemann, K.O., \& Liu, J. (2017). Canopy leaf water content estimated using terrestrial LiDAR. Agricultural and Forest Meteorology, 232, 152-162. 


\begin{abstract}
Leaf water content (LWC) within plant canopy plays an important role in light penetration and scattering, thus affecting reflectance simulation with radiative transfer models. It is also of key importance for the distribution of other plant biochemical parameters, fire propagation simulation and habitat suitability evaluation. Although passive remote sensing techniques have been widely applied to estimate LWC, they are unable to retrieve the LWC vertical distribution within canopy. In this paper we investigated the applicability of the full-waveform terrestrial laser scanning data (TLS) to estimate the LWC vertical distribution within the canopy of individual plants. A modified skewed Gaussian function that accommodates the nonlinear nature of the system was proposed to perform a decomposition on the full-waveform data. The amplitude, the backscatter cross-section, and the backscatter coefficient were assessed to estimate LWC, respectively. Our results showed that the backscatter coefficient had the strongest correlation with LWC $\left(R^{2}=0.66\right)$ for four plant species after an incidence angle correction. Good agreements were achieved between the predicted vertical profile of LWC and the measured vertical profile of LWC with a mean RMSE (root mean square error) value of $0.001 \mathrm{~g} / \mathrm{cm}^{2}$ and a mean MAPE (mean absolute percent error) value of $4.46 \%$. However, the performance of LWC vertical profile estimation varied across species, suggesting the influence of leaf structure other than LWC on waveform features, which should be considered in future studies. Nevertheless, our study successfully demonstrated the feasibility of retrieving LWC vertical distribution within plant canopy from a full-waveform terrestrial laser scanner.
\end{abstract}




\subsection{Introduction}

Leaf water content (LWC) is an important indicator of plant physiological processes (Bowman 1989) such as stomatal conductance, transpiration, photosynthesis and respiration (Yamasaki and Dillenburg 1999), and a key factor affecting forest fire ignition and fire propagation (Chuvieco et al. 2002). The vertical distribution of LWC may also play an important role in the distribution of other plant biochemical parameters (e.g. nitrogen and carbon) (Zhang et al. 2013), fire propagation (Koetz et al. 2008; Saatchi et al. 2007) and habitat suitability evaluation (Scriber 1977a; Tsunoda et al. 2014).

In the past few decades passive remote sensing data have been widely used for LWC estimation at both leaf and canopy levels (Serrano et al. 2000; Tucker 1980). However, the challenge of using this approach is that the reflectance measured from passive remote sensing is complicated by canopy structure, soil background and solar illumination (Ali et al. 2016a; Eitel et al. 2010a; Wessman 1994). Additionally, passive remote sensing cannot capture the vertical LWC distribution within the canopy. Radiative transfer models can successfully simulate light penetration and scattering, with the assumption that the vertically homogeneous canopy extends to two layers including an understory layer (Liu et al. 2015; Verhoef 1984; Yi et al. 2013). However, plant biophysical and biochemical properties (e.g. leaf area index, chlorophyll content and LWC) vary vertically within the canopy greatly affecting light penetration and scattering (Ciganda et al. 2008; Tang et al. 2012; Wang and Li 2013). Using vertically homogeneous radiative transfer models for simulating directional reflectance by using only the average values of LWC within the plant canopy may lead to significant bias (Wang and Li 2013).

Terrestrial laser scanning (TLS) data can potentially overcome such intrinsic limitations of passive remote sensing data, while being capable of providing vertical information (Calders et al. 2014; Côté et al. 2012; Hosoi and Omasa 2009). There are two different techniques used in LiDAR (light detection and ranging) systems - discrete return and full-waveform. Discrete return LiDAR systems identify major peaks in the return signal that represent discrete objects. They provide the geometric coordinates and amplitude of the return power. Both discrete return LiDAR and waveform data have been tested for estimating LWC (Gaulton et al. 2013; Zhu et al. 2015). Compared to discrete return, fullwaveform offers extra information such as echo width (Heinzel and Koch 2011). Fullwaveform also provides more flexibility in the methods that may be used for processing, since the data are not preprocessed and full-waveform output gives the opportunity for users to adjust the processing method to match the application (Fieber et al. 2013; Hancock et al. 2015). Many studies have been carried out on benefits of full-waveform data for object classification. Heinzel and Koch (2011) explored full-waveform parameters for tree species classification with an overall accuracy of 57\%. Alexander et al. (2010) demonstrated the advantage of using full-waveform attributes in urban classification. Reitberger et al. (2008) developed a methodology for tree species 
classification using full-waveform features, leading in the best case to an overall accuracy of $85 \%$ in a leaf-on situation and $96 \%$ in a leaf-off situation.

A decomposition of the waveform is needed to be performed in order to obtain the fullwaveform parameters. One approach, a Gaussian decomposition, is commonly used to decompose a waveform into a sum of echoes (Mallet and Bretar 2009). More specifically, the range, amplitude and width of each echo can be extracted by applying a decomposition. Gaussian decomposition was developed initially to apply to 'Laser Vegetation Imaging Sensors' (LVIS) waveforms (Hofton et al. 2000). It has been widely applied in various fields, such as vegetation detection (Höfle et al. 2012), tree species classification (Yao et al. 2012) and roughness mapping (Hollaus et al. 2011). A complication is that some LiDAR systems have a nonlinear response to high energy returns resulting in asymmetric curves (Hartzell et al. 2015): a constraint also relevant to the TLS (RIEGL VZ-400) used in this study. Due to the nonlinearity in the detector electronics, there is a long trailing edge at the right side of the waveform (Hancock et al. 2015), meaning that a standard Gaussian approach is not suitable for this type of waveform decomposition. Hartzell et al. (2015) proposed an empirical system response model decomposition from a series of observations using calibrated reflectance targets, and presented a numerical least squares method for decomposing waveforms. They found that this method decreased decomposition fitting errors for high amplitude returns and reduced range estimation errors on planar surfaces by $17 \%$ over a standard Gaussian model. In this study, we present a modified skewed Gaussian function that introduces asymmetry and therefore reduces range estimation errors. Using this modified skewed Gaussian function, it is possible to retrieve the amplitude and echo width as well as the standard Gaussian function without the need to use reflectance targets.

Following the decomposition, a standard radiometric calibration has been suggested (Wagner 2010) to convert the amplitude and echo width to physically well-defined radiometric quantities (e.g. the backscatter cross-section and backscatter coefficient) proportional to surface reflectance of the target (Höfle and Pfeifer 2007). The backscatter cross-section of a target is equal to the physical cross-sectional area of an idealized isotropic target. It is a measure of the electromagnetic energy intercepted and re-radiated by a target towards the sensor (Wagner et al. 2006). The backscatter coefficient is the backscatter cross-section normalized relative to the illuminated target area (Wagner 2010). The main advantage of using the backscatter coefficient is that the data acquired using different sensors and different conditions can be directly compared (Wagner 2010). Previous studies have highlighted the effectiveness of the backscatter coefficient for classification purposes (Alexander et al. 2010; Mallet et al. 2011). Fieber et al. (2013) derived reflectance values of orange trees from backscatter coefficient of single-peak waveforms, which are consistent with published reflectance values. 
However, estimating LWC from TLS is complicated by the effect of the partial hits at the leaf edge. Partial hits occur when the laser beam partially hits the edge of a leaf so that only a fraction of the laser beam is return back (Eitel et al. 2010a). Thus the intensity from partial hits cannot be directly used to describe the scattering properties of targets before radiometric calibration. Additionally, laser return energy is influenced by the incidence angle between the leaf normal and the laser beam. As the incidence angle deviates from the perpendicular orientation, the returned energy decreases due to the energy dispersion caused by spot spreading (Kaasalainen et al. 2011). This energy decay does not follow the cosine incidence angle law so that radar equation cannot be directly applied to correct the incidence angle effect.

In this paper, we aim to retrieve LWC vertical distribution within plant canopy using fullwaveform TLS. The specific objectives are to: (1) develop a modified skewed Gaussian function to decompose the full-waveform data; (2) evaluate the full-waveform features for estimating LWC; (3) examine the influence of the partial hits and the incidence angle effects on LWC estimation.

\subsection{Materials}

\subsubsection{Measurements of leaf water content}

We selected dwarf schefflera (Schefflera arboricola compacta), weeping fig (Ficus benjamina Twilight), Chinese banyan (Ficus microcarpa), ficus (Ficus benjamina Danielle) and Zanzibar gem (Zamioculcas zamiifolia) for retrieval of simultaneous LWC from TLS measurements. We used 4 plants per species (20 in total). In order to obtain LWC vertical distribution, each plant was divided and stratified into 6-10 vertical layers (with interval of $10 \mathrm{~cm}$ per layer) depending on the height of the plant. At each layer, all leaves were harvested and their total fresh weight and surface area were measured. The leaves were dried in an oven for 72 hours at $65^{\circ} \mathrm{C}$ and their dry weight was measured accordingly. The area of the leaves was measured by a LI-3100C area meter (LI-COR, Lincoln, Nebraska USA). The average LWC $\left(\mathrm{g} / \mathrm{cm}^{2}\right)$ of each layer was computed using the following equation (Danson et al. 1992):

$$
(M W-M D) / A
$$

where $M W$ is the mass of all the fresh leaves in the layer (g), $M D$ is the mass of all the completely dried leaves in the layer (g), and $A$ is the total surface area of the leaves in the layer $\left(\mathrm{cm}^{2}\right)$. In total we obtained 146 samples, of which half were used for modeling and the other half were used for validation. Summary statistics of the measurements are presented in Table 3.1. 
Table 3.1 Summary statistics of the measurements

\begin{tabular}{|c|c|c|c|c|c|}
\hline $\begin{array}{l}\text { Common } \\
\text { name }\end{array}$ & Species name & $\begin{array}{l}\text { Samples } \\
\text { size }\end{array}$ & $\begin{array}{l}\text { Mean LWC } \\
\left(\mathrm{g} / \mathrm{cm}^{2}\right)\end{array}$ & $\begin{array}{l}\text { Max } \\
\text { LWC } \\
\left(\mathrm{g} / \mathrm{cm}^{2}\right)\end{array}$ & $\begin{array}{l}\text { Min } \\
\mathrm{LWC} \\
\left(\mathrm{g} / \mathrm{cm}^{2}\right)\end{array}$ \\
\hline $\begin{array}{l}\text { Dwarf } \\
\text { schefflera }\end{array}$ & $\begin{array}{l}\text { Schefflera arboricola } \\
\text { compacta }\end{array}$ & 37 & 0.027 & 0.031 & 0.024 \\
\hline Weeping fig & $\begin{array}{l}\text { Ficus benjamina } \\
\text { Twilight }\end{array}$ & 26 & 0.018 & 0.020 & 0.015 \\
\hline $\begin{array}{l}\text { Chinese } \\
\text { banyan }\end{array}$ & Ficus microcarpa & 30 & 0.023 & 0.025 & 0.013 \\
\hline Ficus & $\begin{array}{l}\text { Ficus benjamina } \\
\text { Danielle }\end{array}$ & 28 & 0.017 & 0.020 & 0.010 \\
\hline Zanzibar gem & $\begin{array}{l}\text { Zamioculcas } \\
\text { zamiifolia }\end{array}$ & 25 & 0.048 & 0.053 & 0.035 \\
\hline Pooled data & & 146 & 0.026 & 0.053 & 0.010 \\
\hline
\end{tabular}

\subsubsection{Terrestrial laser scanning data}

The TLS data used in this study was a time-of-flight scanner RIEGL VZ-400 (Riegl LMS $\mathrm{GmbH}$, Horn, Austria). It employs a short wave infrared $(1550 \mathrm{~nm})$ laser, known to be sensitive to LWC (Tucker 1980). The system has a beam divergence of $0.35 \mathrm{mrad}$, a range accuracy of $5 \mathrm{~mm}$ and an effective measurement rate of 122,000 measurements/s. The pulse energy follows a Gaussian distribution within the laser beam. RIEGL VZ-400 provides both discrete returns and waveform outputs allowing users to adjust the processing methods. The data were acquired in a high speed mode with a pulse repetition rate (PRR) of $300 \mathrm{kHz}$ and an angular step of $0.035^{\circ}$ at a distance of $3 \mathrm{~m}$. The zenith angle of scanning was between $100^{\circ}$ and $130^{\circ}$. The point spacing at this distance is about $2 \mathrm{~mm}$. A typical leaf area is between 20 and $30 \mathrm{~cm}^{2}$, so the number of points on a single leaf is around 500. The waveform data were exported from the instrument using RiWAVELib wfm library(RIEGL Laser Measurement Systems, 2014) and processed in Python.

\subsection{Methods}

\subsubsection{Waveform decomposition}

\subsubsection{Modified Gaussian function}

Due to the asymmetric characteristics of the waveform (Fig. 3.1), the TLS waveform data were decomposed by fitting a series of modified skewed Gaussian functions. 


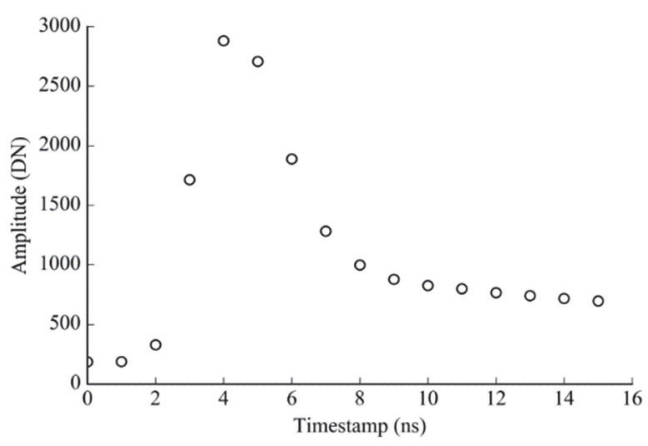

Figure 3.1 A waveform generated from a RIEGL VZ-400 single laser return for extended targets with high reflectance.

A standard Gaussian function describing a single-peak waveform is shown in the following equation (Wagner et al. 2006):

$$
y=A_{0} \exp \left(-\left(t-\mu_{0}\right)^{2} / 2 s_{0}^{2}\right)+n_{0}
$$

where $A_{0}$ is the amplitude, $s_{0}$ is the standard deviation and $n_{0}$ is the noise, $\mu_{0}$ is the peak position and $\mathrm{t}$ is the timestamp.

In order to describe the asymmetric waveform, we firstly replaced the standard deviation $s_{0}$ with a well behaved function $s(t)$ to model the asymmetry (Stancik and Brauns 2008). The standard deviation of the function is allowed to vary:

$$
s(t)=2 s_{0} /\left(1+\exp \left(a\left(t-\mu_{0}\right)\right)\right)
$$

The parameter $a$ is a measure of asymmetry, negative values skew the spectrum toward higher wave numbers while positive values skew it toward lower wave numbers (Stancik and Brauns 2008). When $a$ is zero, Eq. 3.3 becomes a standard Gaussian function as in Eq. 3.2.

Secondly, we adjusted the noise parameter $n_{0}$ to fit the right trailing edge, replacing it with a function:

$$
n(t)=n_{0} \exp (b \mu(t))
$$

where $b$ is a scaling parameter and $\mu(t)$ equals 0 when $t$ is equal or smaller than $\mu 0, \mu(t)$ equals 1 when $t$ larger than $\mu_{0}$. The amplitude $A_{0}$ is also replaced by a function $A(t)$ to make the function continuous at $\mu_{0}$ :

$$
A(\mathrm{t})=\mathrm{A}_{0}-n(\mathrm{t})
$$


For radiometric calibration, we used the value $A_{0}-n_{0}$ as amplitude, and $s_{0}$ as the standard deviation in order to calculate the backscatter cross-section and the backscatter coefficient as a standard Gaussian function (Wagner 2010).

\subsubsection{Fitting algorithm}

Following the decomposition method developed by Hofton et al. (2000), all LiDAR waveforms were modeled by the aforementioned skewed Gaussian function instead of the standard Gaussian function. Firstly, a Gaussian smoothing was performed with a predetermined half-width from the observed waveform in order to isolate the inflection points. Secondly, the initial position and pulse width were estimated based on the inflection points, and the amplitude was estimated using a non-negative least square method. Finally, a constrained least square technique was applied to model the waveform using the skewed Gaussian function. The amplitude was limited to positive values, pulse width was constrained between $0 \mathrm{~ns}$ and $3 \mathrm{~ns}$, while $a$ was constrained between -1 and 1 . The constrained least square technique was used to minimize the sum of the squared differences between the model and the data.

\subsubsection{Radiometric calibration}

The physical principles of the ranging process in LiDAR systems can be described with the same principles as radar systems but at shorter wavelengths (Briese et al. 2008). Before applying the radar equation, a calibration table is used to compensate the nonlinear behavior of the received amplitude for the stronger signal provided by the RiWAVELib wfm. For Gaussian scatterers, considering system variables and additional power losses, the cross-section may be calculated as (Wagner 2010):

$$
\sigma_{i}=C_{c a l} R_{i}^{4} \hat{P}_{i} s_{p, i}
$$

where $\sigma_{i}$ is the cross-section of the $\mathrm{i}^{\text {th }}$ target, $R$ is the range, $\hat{P}_{i}$ is the amplitude and $s_{p, I}$ is the standard deviation. $C_{c a l}$ is the calibration constant:

$$
C_{c a l}=4 \pi \beta_{t}^{2} / \eta_{s s y} \eta_{a t m} D_{r}^{2} \hat{S}_{s}
$$

where $\eta_{s y s}$ is the system transmission factor, $\eta_{\text {atm }}$ is the atmospheric transmission factor, $\beta_{t}$ is the transmitter beam width, $D_{r}$ is the aperture diameter of the receiver optics and $\hat{S}$ and $s_{s}$ are the intensity and pulse width of the transmitted laser pulse, which are assumed to be constant in this study.

In order to obtain the calibration constant, an external Lambertian reference target with known reflectance is needed to estimate the backscatter cross-section (Wagner et al. 2006). We used the reference reflector (Spectralon from Labsphere, Inc.) with a reflectance of 0.17 . The theoretical cross-section of the reflector $\sigma_{r e f}$ is given by: 


$$
\sigma_{r e f}=0.17 \pi R^{2} \beta_{t}^{2}
$$

and the estimate of the calibration constant is:

$$
C_{c a l}=0.17 \pi \beta_{t}^{2} / \mathrm{R}^{2} \hat{P}_{r e f} s_{p, r e f}
$$

where $\hat{P}_{\text {ref }}$ and $s_{p, r e f}$ are the observed mean amplitude and standard deviation of the reflector.

The backscatter coefficient $\gamma$ is the cross-section normalized by the illuminated target area $\left(\mathrm{m}^{2} \mathrm{~m}^{-2}\right)$ (Wagner 2010):

$$
\sigma_{0}=\sigma / A_{i}
$$

where $A_{i}$ is the illuminated target area.

For an extended target that is larger than the footprint size of the laser beam, the illuminated area is equal to the laser's footprint area $A_{l f}$. Such a target obviously produces only a single echo.

$$
\sigma_{0}=\sigma / A_{l f}
$$

Eq. 3.11 only applies on full hits, where the full laser beam hits an extended target which triggers a single return. However, at the edge of a leaf there are many partial hits where only a part of the laser beam hits the leaf. As a consequence, the collision area of the laser beam and the leaf is not identical with the footprint. There are three cases of partial hits. Firstly, when the laser beam is split on a leaf edge and the rest of the energy triggers another return. Secondly, when most of the laser beam hits on a leaf, the rest of the energy is too weak to trigger another return. Lastly, the rest of the energy exits the canopy without another return after a partial hit on a leaf edge. In the first case, the partial hits can be identified as multiple returns. In the second and third cases, there is no second return that can be identified. We have only considered the partial hits in the first case. Because in the second case, the attenuation of the first return is high and the amount of remaining transmitted energy is trivial, we treated them as full returns. As for the third case, the position of the plants in this study were lower than the laser scanner, so the zenith angle of all the laser beams was larger than $90^{\circ}$. Therefore, the LiDAR energy could always hit the ground, and this energy component is obviously included in the first two cases. The backscatter cross-section is the effective area of collision of the laser beam and the target, taking into account the directionality and strength of reflection, as described in (Wagner et al. 2008a):

$$
\sigma_{i}=\left(4 \pi / \Omega_{i}\right) \rho_{i} A_{i}
$$

where $\Omega_{i}$ is the scattering solid angle, $\rho_{i}$ is the reflectivity of the target, and $A_{i}$ is the effective area of collision. For multi-echo waveform, $A_{i}$ is not equal to the footprint area $A_{l f}$. 
Assuming the scattering solid angle $\Omega_{i}$ and reflectivity $\rho_{i}$ of the target are the same for points on the same leaf, the collision area can be obtained using the ratio of the query point and its neighborhood full hit points:

$$
A_{i} / A_{n}=\sigma_{i} / \sigma_{n}
$$

where $\sigma_{n}$ is the mean cross-section of neighborhood full hit points, and $A_{n}$ is the mean collision area of neighborhood full hit points, which is equal to the mean footprint area of these points. So in this step we used the K nearest neighbor (KNN) algorithm to search for full hit points (single returns) close to query partial hit points identified as multiple returns. Therefore, it was possible to obtain the collision area of the partial hit points using Eq. 3.13 The backscatter coefficient was calculated by dividing cross-section by the actual collision area instead of the footprint area for partial hits.

\subsubsection{Correcting for the incidence angle effect}

The backscatter coefficient decreases with increasing incidence angle (Wagner 2010). In this study, we used the reference target-based model (Zhu et al. 2015) for incidence angle correction at individual plant level.

\subsubsection{Incidence angle}

The incidence angle is the angle between the surface normal and the laser beam. Thus, the surface normal is required. We used KNN algorithm to search for the neighborhood points of each point. The number of neighbors $(\mathrm{K})$ that we used to estimate the surface normal was 50, and the resulting average size of the search neighborhood around $8 \mathrm{~mm}$. Then the surface normal was estimated based on the analysis of the eigenvectors and eigenvalues of a covariance matrix from the nearest neighbors of the query point (Gross and Thoennessen 2006). The incidence angle was then calculated as:

$$
\alpha=\arccos (\mathbf{P N} /|\mathbf{P N}|)
$$

where $\alpha$ is the incidence angle, and $\mathbf{P}$ is the laser beam vector from the laser scanner to the surface. $\mathbf{N}$ is the surface normal vector.

\subsubsection{Reference target-based model}

In order to correct the backscatter coefficient, the observed backscatter coefficient at different incidence angles for four panels with different backscatter coefficient at perpendicular angles $\left(\gamma\left(0^{\circ}\right)\right)$ was stored in a look-up table (LUT). The LUT is interpolated to provide $\gamma\left(0^{\circ}\right)$ for any specific incidence angle and observed backscatter intensity, so that $\gamma\left(0^{\circ}\right)$ could be linearly interpolated from the model for a given backscatter intensity recorded by the instrument and the incidence angle. 


\subsubsection{Data analysis}

For statistical analysis, we used half of the plants for modelling and half for validation, with both validation and calibration groups being sampled for each of the 5 species. We evaluated the amplitude, backscatter cross-section and backscatter coefficient to estimate LWC, respectively. In order to explore the full potential of the waveform data, we added the echo width as a co-variable to model LWC, as echo width is affected by the roughness of the object (Lin and Mills 2010). Echo width can potentially help improve the model by differentiating plant species with different roughness.

\subsection{Results}

\subsubsection{Leaf water content measurements}

The LWC measurements varied between species and different layers within plant canopy. As can be observed from Table. 3.1, Z. zamiifolia was considered as an extreme case in this study with very thick leaves and high LWC (about $0.05 \mathrm{~g} / \mathrm{cm}^{2}$ ). We tested our method using both samples with and without the extreme case to assess the prediction ability of the waveform parameters on species with very high LWC.

\subsubsection{Waveform decomposition}

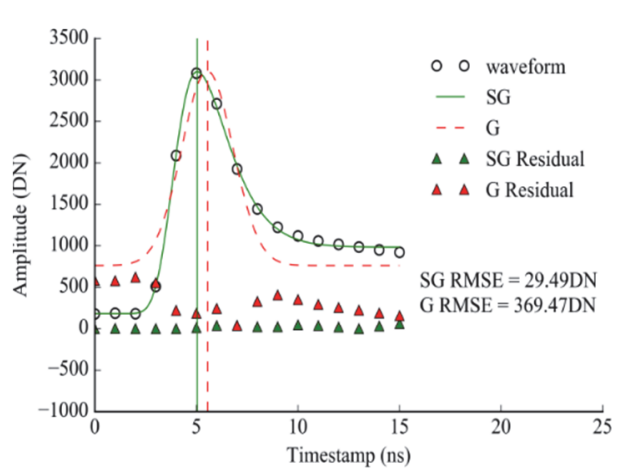

h

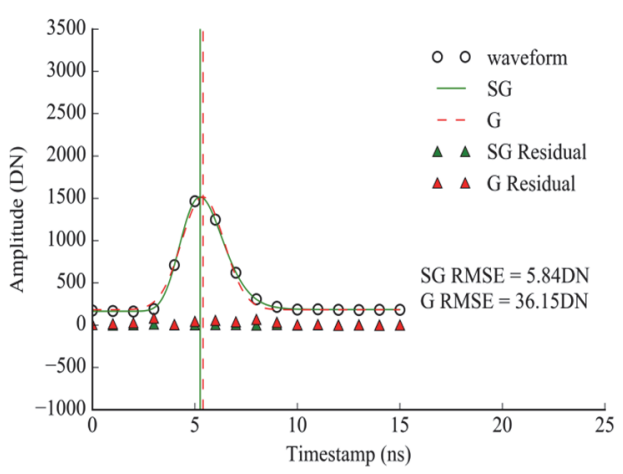

Figure 3.2 Waveform decomposition of a single high amplitude (a) and low amplitude (b), echo with the standard Gaussian model (G) and the modified skewed Gaussian (SG) model (the vertical lines are peak position)

The RMSE and residuals values were calculated between the observed waveform and the fitted models. The observed waveform is the waveform exported from the scanner which is composed of digitized samples with exact timestamp and amplitude. Compared to the standard Gaussian model, the RMSE (root mean square error) and residuals values of the decomposition results were significantly reduced using the modified skewed Gaussian model. The results are presented in Fig. 3.2. As can be observed from the figure, for low 
amplitude echoes, the RMSE decreased from $36.15 \mathrm{DN}$ to $5.84 \mathrm{DN}$. The accuracy of high amplitude echo modeling was also much higher with the skewed Gaussian model.

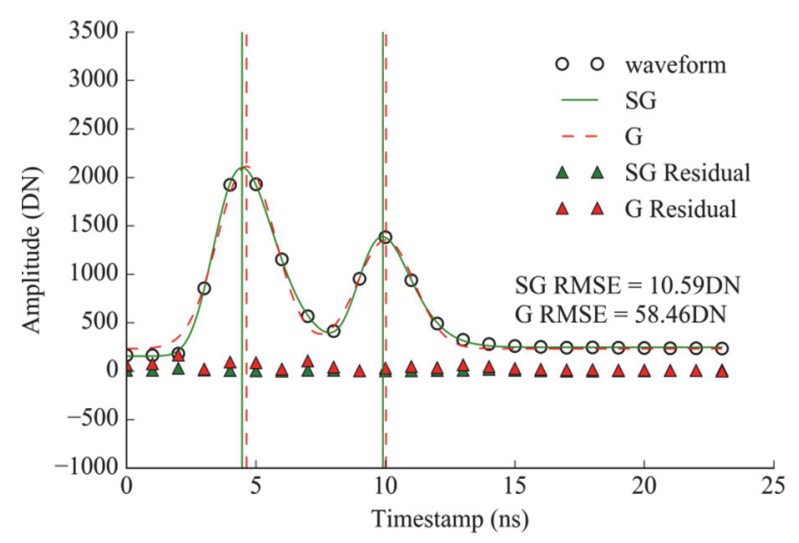

Figure 3.3 Waveform decomposition of a multiple echo with the standard Gaussian model (G) and the skewed modified Gaussian (SG) model (the vertical lines are peak position)

The decomposition of a multiple echo generated from a vegetation canopy using the standard Gaussian function and the skewed Gaussian function is shown in Fig. 3.3. Gaussian decomposition peak positions were delayed again due to the trailing edge of high amplitude targets, while the skewed Gaussian decomposition showed a significantly lower RMSE (10.59 DN). Table. 3.2 shows the decomposition statistical comparison for a number of echoes generated from a scan of a sample plot with several trees. As can be observed form the table, the average RMSE of both single and double echoes were largely improved by using the skewed Gaussian decomposition.

Table 3.2 Mean $R^{2}$ and RMSE values of waveform decomposition comparison between Gaussian decomposition $(\mathrm{G})$ and skewed Gaussian decomposition (SG)

\begin{tabular}{llllll}
\hline Echoes & $\begin{array}{l}\text { Sample } \\
\text { number }\end{array}$ & Mean $R^{2}$ & & \multicolumn{2}{l}{ Mean RMSE (DN) } \\
\hline 1 & G & SG & G & SG \\
2 & 3782 & 0.96 & 0.99 & 121.49 & 16.73 \\
\hline
\end{tabular}

The results of decomposition for reflectance panels are shown in Fig. 3.4. Gaussian decomposition led to notable range discrepancies between target panels with different reflectance. The range discrepancy increased as the reflectance increased. In comparison, the range differences of the skewed Gaussian decomposition were minimized, especially for the three panels with medium and low reflectance. The range discrepancy for the panel with $99 \%$ reflectance has been largely reduced by using the skewed Gaussian 
decomposition, while there were still notable differences. However, it was not in the scope of this research, since our main targets in this study are leaves with low amplitude.

a

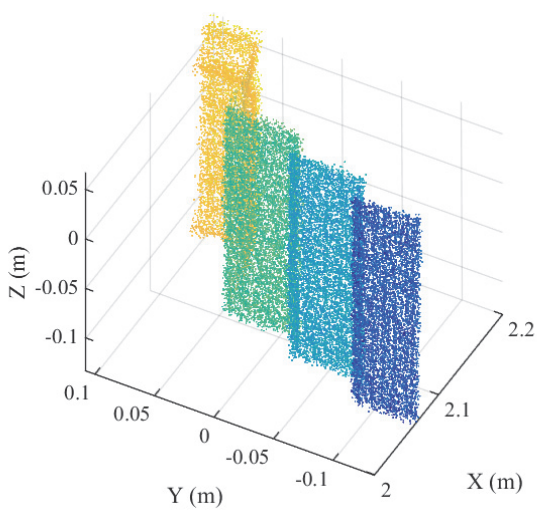

b

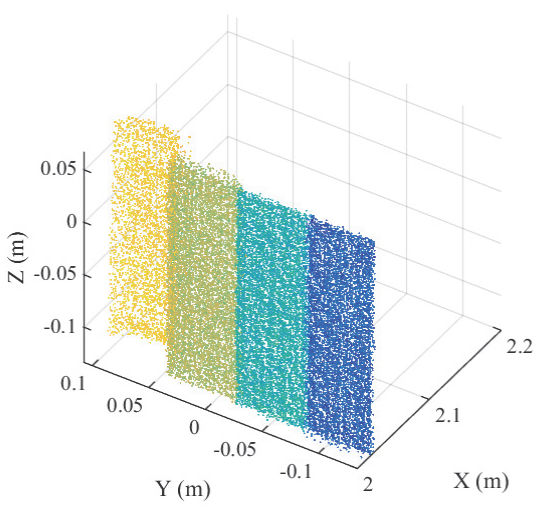

Figure 3.4 Results of decomposition for four reflectance panels (Spectralon from Labsphere, Inc. with reflectance of $99 \%, 61 \%, 34 \%$ and $17 \%$, from left to right). (a) Gaussian decomposition, and (b) Skewed Gaussian decomposition

\subsubsection{Modeling leaf water content}

Before the incidence angle correction, statistically significant relationships were found between LWC and all the waveform parameters $(P<0.05$, Fig. 3.5). However, none showed a strong relationship with LWC (amplitude, backscatter cross-section and backscatter coefficient). All the values of the three parameters declined as LWC increased, while the $R^{2}$ value $(0.38)$ between the backscatter coefficient and LWC was higher than the other two parameters. The backscatter coefficient is still the most sensitive parameter to LWC change $\left(R^{2}=0.50\right)$. However, none of the relationships was strong enough to build a model for predicting LWC of the pooled data. As the backscatter coefficient had a stronger relationship with LWC, it was selected to correct the incidence angle effect for modelling LWC. Fig. 3.6 shows the relationship between the backscatter coefficient and LWC after correcting for the incidence angle effect. Strong relationship $\left(R^{2}=0.66\right)$ emerged, indicating the effectiveness of incidence angle correction. A linear model was used for the LWC prediction without the extreme case.

In addition, we added echo width as a co-variable with the backscatter coefficient to test its usefulness for LWC estimation without the extreme case using a multiple linear regression. The performance of the prediction model was improved. The value of $R^{2}$ increased from 0.66 to 0.70 , and the value of RMSE decreased from $0.0027 \mathrm{~g} / \mathrm{cm}^{2}$ to $0.0025 \mathrm{~g} / \mathrm{cm}^{2}$. 

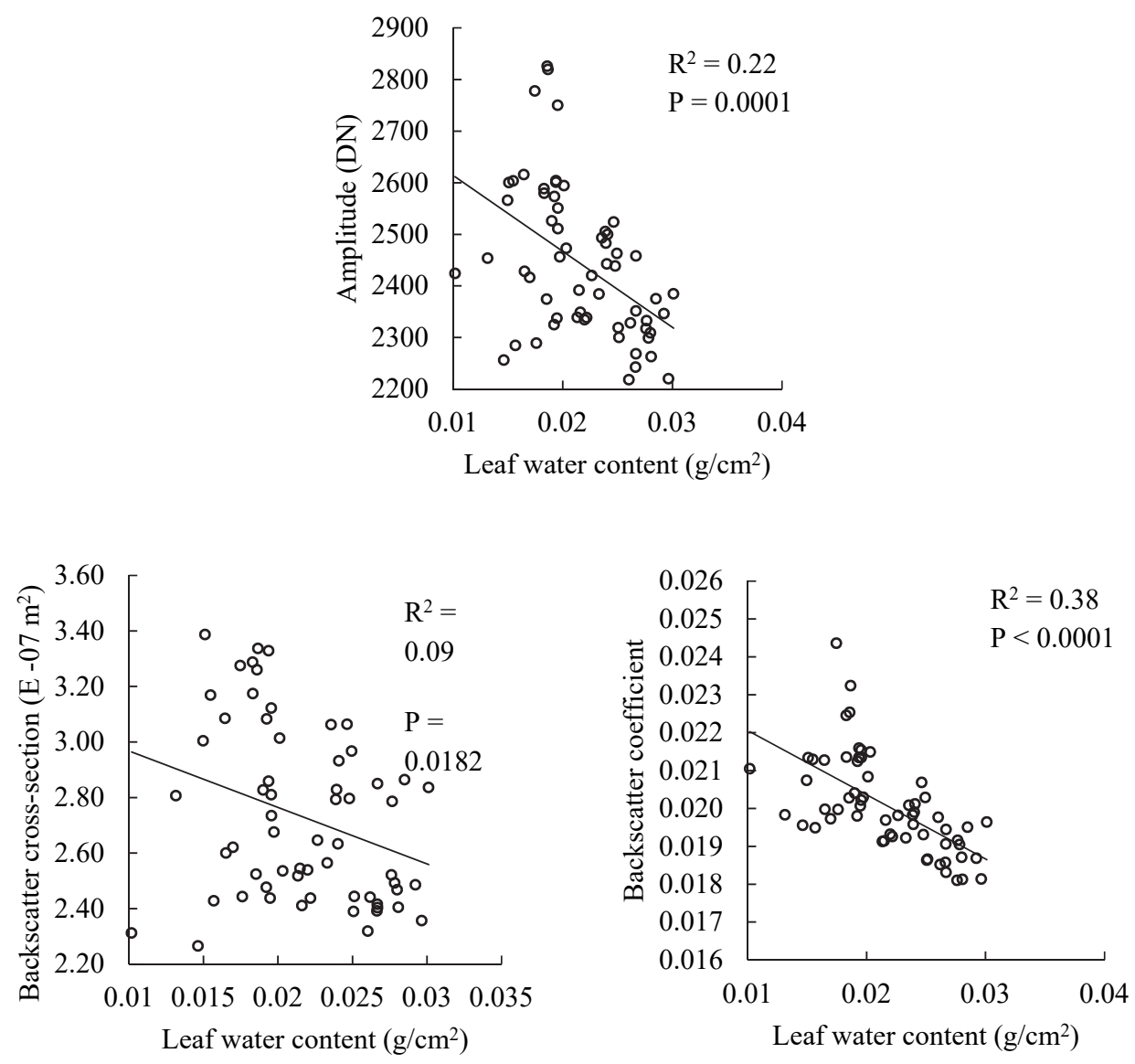

Figure 3.5 Relationships between three waveform parameters (i.e., amplitude, backscatter crosssection, and backscatter coefficient) and LWC before correcting for incidence angle effect

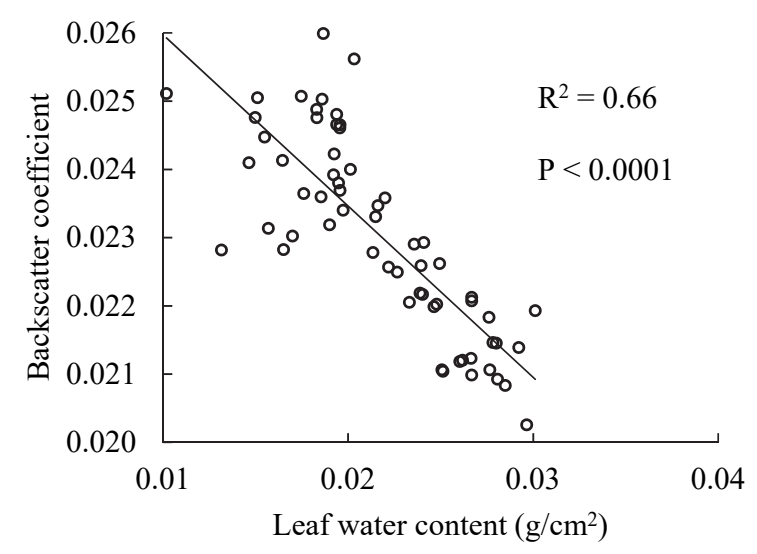

Figure 3.6 Relationships between the backscatter coefficient and LWC after correcting for incidence angle effect 


\subsubsection{Validation of leaf water content estimation}

The observed LWC and predicted LWC from the backscatter coefficient are reported in the scatterplots in Fig. 3.7a. The intercept of -0.001 and slope of 0.956 indicated a high model accuracy, while the $R^{2}$ value of 0.72 and RMSE value of $0.0029 \mathrm{~g} / \mathrm{cm}^{2}$ indicated a high level of precision. In Fig. 7b, the scatterplot of the observed LWC and LWC derived from the prediction model combining the backscatter coefficient and echo width is presented. It can be realized that the slope of 0.756 has decreased from 0.956 (Fig. 3.7a), but the $R^{2}$ value raised from 0.72 (Fig. 3.7a) to 0.85 (Fig. 3.7b). Accordingly the RMSE value declined from $0.0029 \mathrm{~g} / \mathrm{cm}^{2}$ to $0.0016 \mathrm{~g} / \mathrm{cm}^{2}$. In addition, the scattered points were more concentrated to the original 1:1 line (dash line) than in Fig. 3.7a.
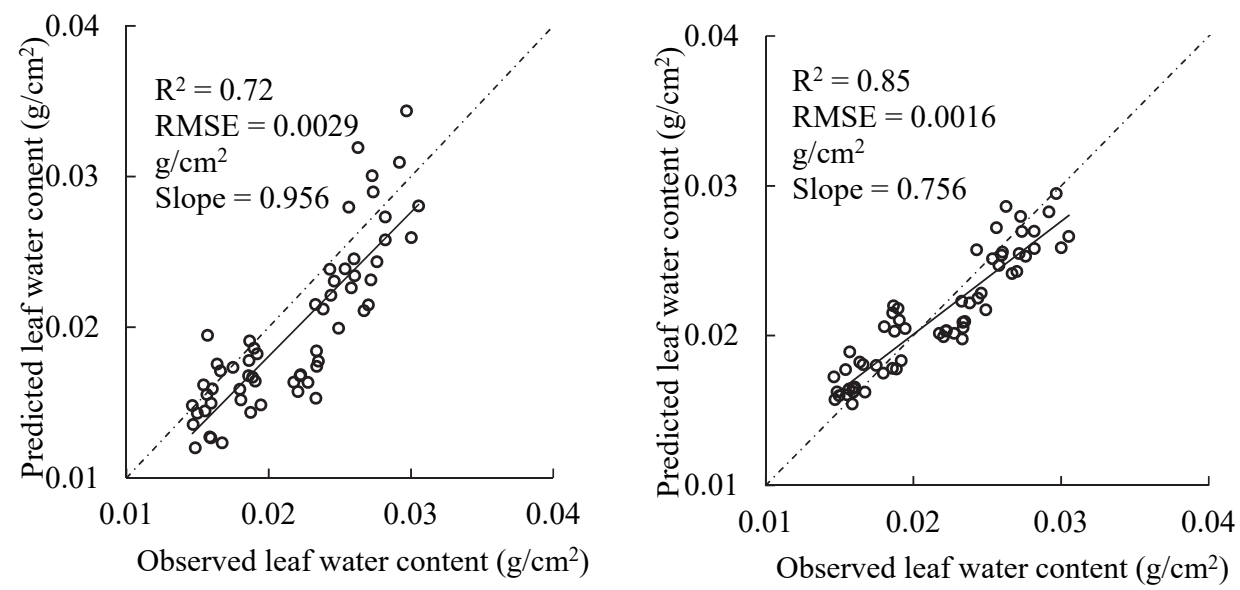

Figure 3.7 Scatterplots of the measured and estimated leaf water content by the backscatter coefficient. (a) prediction without echo width and (b) prediction with echo width

In order to generate LWC vertical profiles, the predicted LWC was compared with the measured LWC at different heights (examples presented in Fig. 3.8). Good agreements were obtained between the predicted and measured LWC vertical profile. The mean MAPE (mean absolute percent error) value of the presented two plants was $4.46 \%$ and the mean RMSE value was $0.001 \mathrm{~g} / \mathrm{cm}^{2}$, indicating a high level of accuracy, while the prediction accuracy varied across species with the RMSE values ranging between 0.0006 $\mathrm{g} / \mathrm{m}^{2}$ and $0.0015 \mathrm{~g} / \mathrm{cm}^{2}$. 

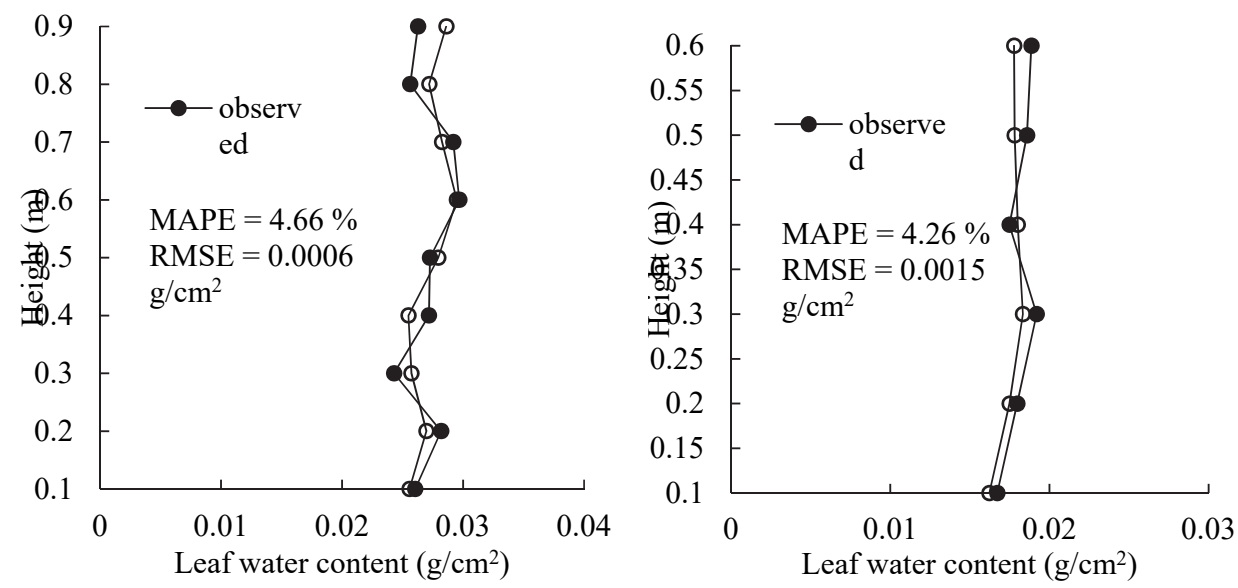

Figure 3.8 Comparison of measured and predicted leaf water content vertical profile (dwarf schefflera, weeping fig)

Fig. 3.9 shows some examples of the LWC vertical profile distribution. The points were distributed in different layers showing the leaf structure distribution along the height. In particular, the points at the top layer of the plants were mostly concentrated at a relatively higher level of LWC with very few points at a lower level, whereas the points at the middle and bottom layers were more evenly distributed at different levels of LWC. At the lower layers, there were a number of points located at very low level of LWC. 
a
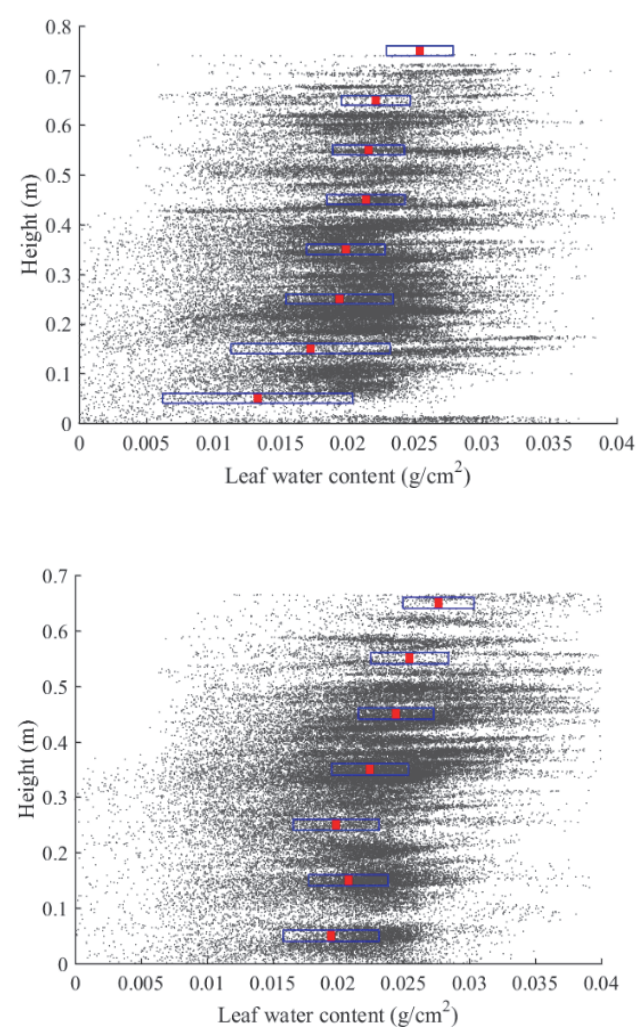

b
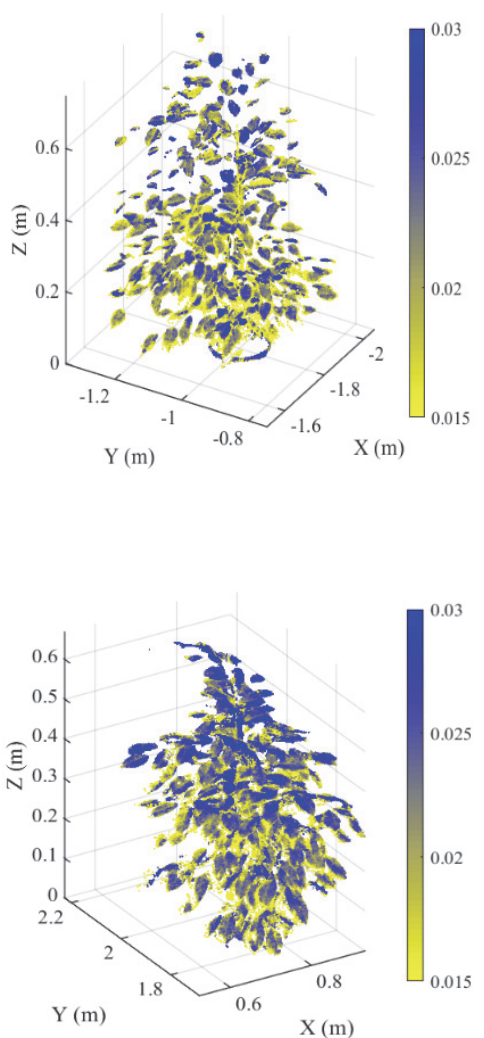

Figure 3.9 Examples of leaf water content distribution of the 2 plant species (weeping fig and ficus). (a) LWC vertical distribution (The red square indicates the mean value and the blue rectangle indicates the standard deviation) and (b) LWC visualization (plants were scanned downward from the TLS)

\subsection{Discussion and conclusion}

In this study, we estimate the LWC vertical distribution within plant canopy using fullwaveform terrestrial laser scanner data. The LWC as a function of plant height was modelled with an acceptable level of accuracy.

Notably, when leaves are fully hydrated, new leaves at the top of a plant have higher water content than older leaves (Mooney et al. 1977). We verified the observation that leaves at the top layers of a plant have high LWC when fully watered (Fig. 3.9). But the differences of LWC along the height were not significant, probably because the height of the plants was too short to show any differences. This phenomenon might be helpful to determine whether a plant is under stress conditions such as drought. Moreover, it has been shown that in some radiative transfer models the vertical heterogeneity of biophysical and biochemical parameters within the canopy largely affects the canopy 
reflectance simulation and inversion (Wang and Li 2013). However, this is very difficult to measure with passive remote sensing data. Using LiDAR techniques, we are able to detect the LWC at different height level. They can provide very useful information for radiative transfer models which incorporate the vertical LWC in the future.

The substantial model fitting errors from the Gaussian decomposition arise from the system nonlinearity effects. This effect led to a right skewed Gaussian echo shape. We adopted and modified a skewed Gaussian function which allowed the echo width to vary with time and the noise level to differ. The modified skewed Gaussian decomposition produced much smaller fitting errors and higher values of $R^{2}$ for both high and low amplitude targets (Fig. 3.2 and Table. 3.1).

Three waveform features, namely the amplitude, the backscatter cross-section and the backscatter coefficient have been tested for modeling LWC. Before the incidence angle correction, all the three parameters were significantly correlated with LWC, though none of them had a high enough correlation to accurately predict LWC. This was mainly due to the variation of the incidence angle effect. The high correlation achieved after incidence angle correction indicates the effectiveness of the reference target based method. This method is supposed to be more effective for near-Lambertian targets, since the specularity of a leaf could be very different from the Spectralon. However, the incidence angle between leaves and laser beams was mostly off-nadir due the orientation of the scanning zenith angle and the leaf angles in this experiment. According to the Phong reflection model, the specular fraction of the intensity was very small when the incidence angle was larger than $10^{\circ}$ (Ding et al. 2013). As a result, the diffuse intensity was the dominant intensity. Therefore, the reference targets based method has significantly improved the results. In a forest plot when the incidence angle is very small, a reflection model needs to be applied to remove the specular intensity. It is possible to determine the specular and diffuse fraction using a reflection model with a sample of near-nadir points.

In Fig. 3.9, the visualization shows the LWC of each point. The low values of LWC indicated high laser energy, as water absorbed the energy. So on some parts of the leaf edge, the corrected backscatter coefficient was slightly higher than the other parts of a leaf. We think this is due to the overcorrection of the incidence angle influence. We found that on the edge of some leaves, the incidence angle was larger due to the curling edge of the leaves. The overestimation of the incidence angle of the leaf edge could lead to overcorrection, leading to a higher backscatter coefficient value.

Among the three parameters, the backscatter coefficient provided the highest value of $R^{2}$ and the lowest value of $P$ (Fig. 3.5). There are two possible reasons for this. Firstly, the backscatter coefficient is normalized relative to the footprint, so the variation of footprint influence has been eliminated. Secondly, the backscatter coefficient is also normalized 
by the illuminated area. Therefore, the influence of attenuated energy on the leaf edge caused by the partial hits from multiple returns has also been minimized. Nonetheless, we have only dealt with the first case of a partial hit where the partial hit has a second return. At plot level, the zenith angle of the laser beams could be smaller than $90^{\circ}$, so after a partial hit, the rest of the energy might exit the canopy without a return. Such partial hits which cannot be neglected are more difficult to be identified: this requires further investigation at plot level.

After the correction of the incidence angle effect with a reference targets-based model, the correlation between backscatter coefficient and LWC became higher (Fig. 3.6). It was previously demonstrated that this method is effective for LWC estimation at the leaf level (Zhu et al. 2015). The results of this study have further demonstrated that the method of incidence angle effect correction is also effective for LWC modelling at individual plant level. To the best of our understanding, LWC has little effect on echo width. Echo width exhibits a relative stable behavior when amplitude, range and scan angle vary substantially, while roughness is the most significant factor affecting echo width (Lin and Mills 2010). The reason that adding echo width improved the model might be that the structure among various plant species are different as reflectance is not only affected by LWC, but also plant structure. The performance of $L W C$ vertical profile prediction varied across species (Fig. 3.8) even by adding echo width, indicating the influence of plant structure other than LWC. The newly emerged instruments such as SALCA (Salford Advanced Laser Canopy Analyser) (Danson et al. 2014) with dual-wavelength capability and the hyperspectral TLS currently under development can potentially address the issue by using a ratio of two bands that are similarly affected by the plant structure.

The point density was very high in this study due to the small angular step $\left(0.035^{\circ}\right)$ and close scanning distance $(3 \mathrm{~m})$. There were about 500 points striking a single leaf, so it appears quite feasible to use a $\mathrm{K}$ of 50 to estimate the surface normal of a leaf in the KNN algorithm. Nevertheless, in a forest stand, the point density will be much lower. With the smallest angular step of $0.024^{\circ}$ at a distance of $30 \mathrm{~m}$ (common radius for a forest plot), the distance between two consecutive points is around $1.2 \mathrm{~cm}$. So on a typical leaf with an area of $30 \mathrm{~cm}^{2}$, there are about 20 points. In order to create the plane within a homogenous region, the $\mathrm{K}$ should be reduced to below 20 . Therefore, for a broadleaf forest plot with $30 \mathrm{~m}$ radius, it should still be feasible to estimate the LWC. As for very small leaves (smaller than $10 \mathrm{~cm}^{2}$ ) and long range data, the surface normal approach to calculate incidence angle is not applicable. Using TLS with higher point density and the pulse width to estimate the incidence angle might be a solution in this situation (Pfennigbauer et al. 2013). 


\section{Chapter 4 \\ Retrieval of leaf water content from airborne LiDAR and hyperspectral data*}

\footnotetext{
* This chapter is based on: Zhu, X., Skidmore, A. K., Darvishzadeh, R., Wang, T. (under review). Improved estimation of canopy leaf water content by combining airborne LiDAR and hyperspectral data. ISPRS Journal of Photogrammetry and Remote Sensing.
} 


\begin{abstract}
The accurate estimation of leaf water content (LWC) and knowledge about its spatial variation are important for forest and agricultural management, since LWC provides key information for evaluating plant physiology. Airborne LiDAR data has the advantage of providing canopy structural information, while its intensity data have not yet been explored for LWC estimation. In comparison, hyperspectral data are rich on spectral information but the effects of canopy structure and background variation confound canopy reflectance, introducing error to the retrieval of LWC. Radiative transfer models (RTM) provide a robust approach to combine information obtained by these two different systems. In this study, we sought to improve the accuracy of LWC retrieval using airborne LiDAR and hyperspectral data. Firstly, a canopy height model (CHM) was created using LiDAR point clouds in order to mask vegetation and thereby obtain a variety of soil spectra from hyperspectral imagery. The soil reflectance derived from hyperspectral and LiDAR remote sensing was introduced as an input to the RTMs in order to generate a look-up table. Secondly, forest canopy cover and understory leaf area index were estimated using LiDAR data and used as a priori information to constrain the model inversion. In addition, LiDAR intensity data were also investigated for LWC estimation. The models were validated against field measurements obtained from 26 forest plots and then used to map LWC in the southern part of the Bavarian Forest National Park in Germany. The results show that the introduction of a priori vegetation cover and understory LAI information obtained from LiDAR significantly improved the accuracy of LWC retrieval when inverting INFORM, from an $R^{2}$ of 0.55 and a normalized RMSE (nRMSE) of 0.28 to 0.78 and 0.19 , respectively. The spatial variation in LWC, derived using remote sensing, corresponded strongly with the distribution of broadleaf, needle leaf and mixed forests. Hybrid RTMs such as INFORM, tuned for specific areas through the use of LiDAR and hyperspectral imagery, point to methods potentially useful to upscale RTMs to regional and continental levels.
\end{abstract}




\subsection{Introduction}

Leaf water is an essential component of plant leaves which plays a key role in many physiological processes such as plant growth, photosynthesis, transpiration, and thermal regulation (de Jong et al. 2014; Running and Gower 1991; Ullah et al. 2013) and is a major driver in predicting the susceptibility to fire (Chuvieco et al. 2004). Leaf water content (LWC) is also important as an essential biodiversity variable, having relevance for ecosystem function and ecosystem structure (Skidmore et al. 2015). The quantification of LWC and knowledge about its spatial variation can provide crucial information for assessing forest drought conditions and predicting future forest change associated with climate change (Anderson et al. 1976; Asner et al. 2011).

Remote sensing data can be used to retrieve leaf properties, including LWC, across a wide range of spatial and temporal scales (Zarco-Tejada et al. 2003). A number of studies have successfully demonstrated the feasibility of retrieving leaf biochemical variables from passive remote sensing data through empirical approaches as well as the inversion of radiative transfer models (RTM) (Ceccato et al. 2001; Cheng et al. 2012; Cheng et al. 2006; Colombo et al. 2008; Mirzaie et al. 2014). Empirical correlations are usually established by linking leaf biochemical variables to reflectance using spectral indices (Seelig et al. 2008; Tong and He 2017), artificial neural networks (Dawson et al. 1999; Mutanga and Skidmore 2004), or partial least square regression (Asner et al. 2015; Atzberger et al. 2010). The main disadvantage of empirical approaches is that they need field observations for model calibration and that the established model may be highly dependent on site and sampling conditions (Cheng et al. 2012; Darvishzadeh et al. 2012). In contrast, RTMs simulate physical processes describing the interaction of photons and vegetation components, and their inversion allows the retrieval of vegetation biochemical and biophysical variables, thus offering advantages in robustness and transferability compared to empirical models (Colombo et al. 2008; Darvishzadeh et al. 2008b; Houborg et al. 2009).

At the canopy level, the link between leaf biochemical variables and reflected radiation is often complicated by canopy structure (e.g. canopy cover, leaf area index (LAI)) (Colombo et al. 2008; Niemann et al. 2012; Ollinger 2011; Wang et al. 2017). According to Fourty and Baret (1998), a large variation in LAI may weaken the relationship between LWC and spectral reflectance. Zarco-Tejada et al. (2003) demonstrated the dependency of water-related optical indices on LAI. Cheng et al. (2006) showed that the retrieval of LWC using RTMs was significantly influenced by vegetation canopy architecture causing over or under estimations.

In addition to the complications arising from canopy structure, background reflectance forms a source of variability in canopy reflectance (Houborg et al. 2009; Jacquemoud 1993). Caetano and Pereira (1996) found that in sparse forest the spectral characteristics 
of the background have a significant contribution to the reflectance of mixed targets of overstory and background. Zarco-Tejada et al. (2004) indicated that soil background dominated the bidirectional reflectance signature in open crop canopies. Cheng et al. (2006) simulated canopy reflectance with three different soil background types using an RTM and showed that the variation in soil background reflectance had a significant effect on canopy reflectance and contributed to the error when retrieving LWC. Eriksson et al. (2006) evaluated the impact of understory on remotely sensed LAI, indicating that the reflectance values vary by up to $\pm 18 \%$ in the red and up to $\pm 10 \%$ in the near infra-red region of the spectra due to the understory and the resulting variation in LAI due to the understory vegetation was found to be 1.6 units on average. Colombo et al. (2008) demonstrated that spatial variability in background reflectance could produce significant variation in canopy reflectance data, especially when the canopy is sparse. Moreover, a sensitivity analysis using the PROSAIL radiative transfer model (Jacquemoud et al. 1995) showed that for sparse canopies, soil reflectance accounted for $4-34 \%$ of the variation in canopy reflectance (Xiao et al. 2014).

A common challenge when using RTMs is the ill-posed problem in the model inversion, as is well documented in a number of studies (Combal et al. 2003; Koetz et al. 2005). The ill-posed problem is mainly caused by the under-determined nature of the modeling schemes (Jacquemoud et al., 1995). Different parameter combinations may yield almost the same spectral signatures (Darvishzadeh et al. 2008a; Jacquemoud 1993). The use of prior knowledge of the model input parameters may help overcome this problem during inversion and improve the estimation of vegetation variables (Combal et al. 2002; Darvishzadeh et al. 2008b). Darvishzadeh et al. (2008a) examined the effect of soil background brightness on LAI retrieval and suggested that using prior knowledge about soil background was important when estimating vegetation variables. Dasgupta et al. (2009) showed that the use of prior information improved fuel moisture content estimation by $18-27 \%$ in a grassland when employing the PROSAIL model. Xiao et al. (2014) indicated that the usage of prior knowledge of canopy structure when inverting an RTM was key to accurately estimating leaf biochemical variables, especially with sparse canopy cover. A number of studies utilizing RTMs have incorporated soil or background reflectance as a separate layer (Atzberger 2000; Kötz et al. 2004; Verhoef and Bach 2003, 2007). However, soil reflectance is often introduced as fixed background reflectance, or if it is considered, then only a small number of different soil types from existing soil type maps have been used (Laurent et al. 2011; Meroni et al. 2004; Yang et al. 2011): this will not fully represent the real situation at a large spatial scale. Prior information of background reflectance and canopy structure is usually collected from field measurements (Ali et al. 2016b; Rautiainen 2005; Yang et al. 2011), though such in situ sampling is labor intensive and time-consuming, especially in remote areas.

An alternative way to obtain information on background reflectance and canopy structural variables more efficiently at large spatial scale is to use airborne Light Detection and 
Ranging (LiDAR). LiDAR data, which provide three-dimensional (3D) information, have been successfully used to estimate vegetation structural properties such as canopy height, canopy cover and LAI (Fieber et al. 2015; Korhonen et al. 2011; Solberg et al. 2009). In addition to structural information, LiDAR also provides radiometric information as backscatter intensity. Intensity data were found to be particularly useful for identify different tree species (Heinzel and Koch 2011; Ørka et al. 2009). But the use of intensity for the estimation of essential biodiversity variables is still limited. The main reason is that LiDAR intensity is affected by many factors including atmospheric conditions, the distance between the sensor and the target and the incidence angle between the laser beam and the target surface normal (Höfle and Pfeifer 2007). Eitel et al. (2010a) used a green band TLS $(532 \mathrm{~nm})$ to measure leaf chlorophyll content, and showed that the green band backscatter intensity is strongly correlated with leaf chlorophyll content $\left(R^{2}=0.77\right)$. Zhu et al. (2015) corrected the incidence angle effects on the intensity data of a TLS, showing that LWC could be estimated with a good accuracy $\left(R^{2}=0.76\right)$. However, directly observing LWC from airborne LiDAR has not been explored.

The integration of LiDAR and hyperspectral data offers the potential to solve key issues related to the effects of canopy structure on the reflectance spectra and the inversion of RTMs by providing prior information (Niemann et al. 2012). Koetz et al. (2007) showed that the introduction of prior information derived from LiDAR data in RTM inversion significantly improved the estimation accuracy of canopy cover compared to an estimation based solely on spectral information. Cao et al. (2012) decomposed the reflectance of spectral scene components using LiDAR. The result was then used as an input in a simplified Li-Strahler geometric-optical model (Li et al. 1995). Ma et al. (2014) estimated canopy height using LiDAR data to parameterize a geometric-optical mutualshadowing (GOMS) model for the retrieval of LAI in a natural forest. To our knowledge, no study has used LiDAR in combination with hyperspectral data to retrieve background reflectance and canopy structure as prior information to retrieve LWC using RTM inversion.

The aim of this study is (1) to demonstrate how airborne LiDAR intensity data may be used for forest LWC estimation, and (2) to obtain a priori information of soil spectra, canopy cover and understory LAI from the combination of LiDAR and hyperspectral data, and use this information to improve the accuracy of LWC estimation through the inversion of a canopy radiative transfer model.

\subsection{Materials}

\subsubsection{Study area}

The study area is located in the Bavarian Forest National Park in southeastern Germany and covers an area of 24,250 hectares of forest (Fig. 4.1). The elevation ranges from 600 
to $1453 \mathrm{~m}$. The natural forest ecosystems of the Bavarian Forest National Park vary according to altitude: there are alluvial spruce forests in the valleys, mixed mountain forests on the hillsides and mountain spruce forests in the high areas. Dominant tree species are Norway spruce (Picea abies) (67\%) and European beech (Fagus sylvatica) (24.5\%), with some white fir (Abies alba) (2.6\%), sycamore maples (Acer psudoplatanus) (1.2\%) and mountain ash (Sorbus aucuparia) (3.1\%) (Heurich et al. 2010).

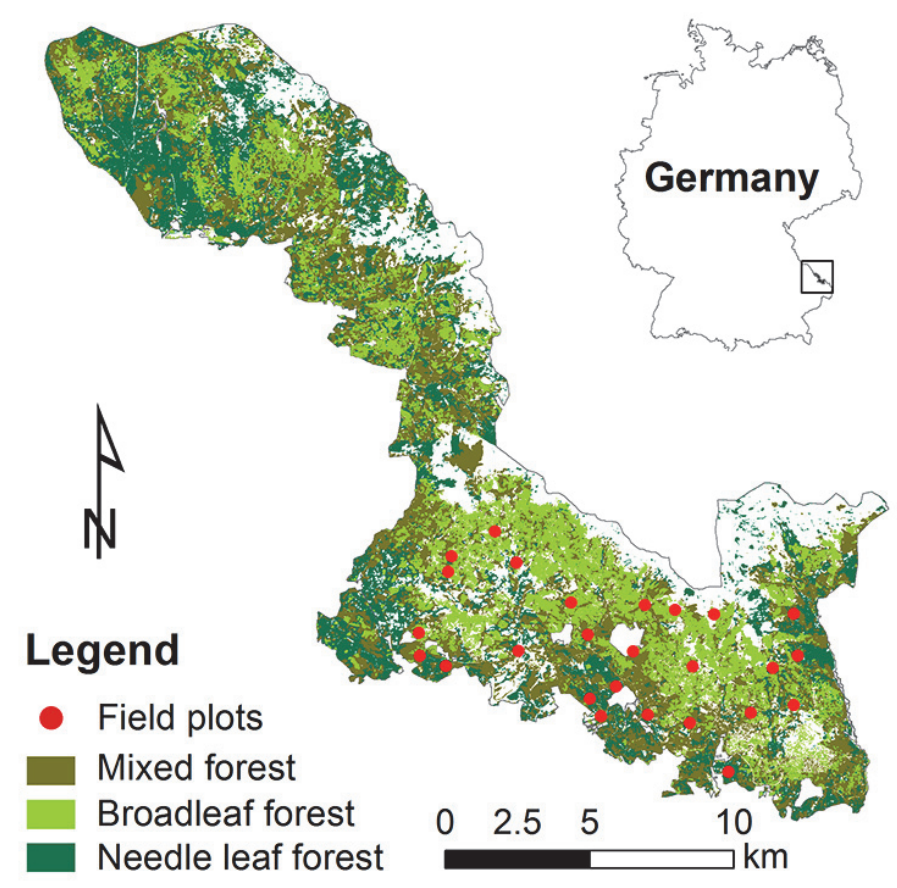

Figure 4.1 The location of the study area in Germany and the distribution of sample plots in the southern part of the Bavarian Forest National Park

\subsubsection{Field data collection and laboratory analysis}

The fieldwork was carried out between July 11 and August 23, 2013, using a stratified random sampling design. A land cover map with the classification of broadleaf, needle leaf and mixed forest including stand age (i.e. mature, medium and young) was provided by the Bavarian Forest National Park for analysis. To incorporate the heterogeneity and variation in canopy structure, 26 plots ( 7 broadleaf, 6 needle leaf, and 13 mixed stands) were randomly selected. Each plot was $30 * 30 \mathrm{~m}$ in size. Within each plot, leaf samples were taken from the branches of one to three dominant tree species depending on the homogeneity. Each sample consisted of at least 20 leaves taken from the top of each selected tree using a crossbow. For each leaf sample, leaf water weight (g) and leaf dry mass $(\mathrm{g})$ were measured from fresh and oven-dried $\left(65^{\circ}\right.$ for $\left.48 \mathrm{~h}\right)$ mass in the laboratory. 
The one-sided area of each leaf sample was computed by multiplying the number of leaf pixels by the pixel area. Leaf pixels were distinguished on the scanned image by performing a segmentation using the Matlab software (MATLAB, The MathWorks Inc., USA, 2016). Then LWC $\left(\mathrm{g} / \mathrm{cm}^{2}\right)$ and leaf dry mass per area $\left(\mathrm{LMA}, \mathrm{g} / \mathrm{cm}^{2}\right)$ were calculated from leaf water weight and dry mass, respectively, along with leaf area. Specific leaf area (SLA, $\mathrm{cm}^{2} / \mathrm{g}$ ) was calculated by dividing the total leaf area of the leaf samples by leaf dry mass.

The canopy structural parameters crown diameter (CD) and stand height (SH) were calculated from the mean $\mathrm{CD}$ and mean height, respectively, of five trees randomly selected in each plot. Tree height, diameter at breast height, and the number of trees for each dominant species were recorded in the field measurments to calculate the species fraction of foliar dry biomass using allometric equations (Widlowski et al. 2003). The plot level LWC was then calculated (Eq. (4.1)) as the mean LWC for each species, weighted by the leaf area fraction, which was calculated from foliar biomass fraction and average specific leaf area for each species (Eq. (4.2)) (Homolova et al. 2013; Wang et al. 2016).

$$
L W C=\sum_{i=1}^{k} l w c_{i} f L A_{i}
$$

where $l w c_{\mathrm{i}}$ represents the average leaf water content of species $i$ within a plot, and $k$ is the number of species within a plot. $f L A_{i}$ is the leaf area fraction of species $i$, which was calculated as

$$
f L A_{i}=\frac{\text { fBiomass }_{i} \text { SLA }_{i}}{\sum_{i=1}^{k} \text { Biomass }_{i} S L A_{i}}
$$

where $f$ Biomass $i$ is the foliar biomass fraction of species $i$, and $S L A_{i}$ is the average specific leaf area for species $i$ within a plot.

At least 16 hemispherical photos at a height of $1.3 \mathrm{~m}$ were taken at each plot from the plot center and $10 \mathrm{~m}$ from the center in all diagonal directions and processed using the Hemisfer software (Hemisfer, Swiss Federal Institute for Forest, Snow and Landscape Research WSL, Switzerland, 2016) to calculate the canopy cover (Thimonier et al. 2010). Hemispherical photos were classified as sky or canopy pixels by applying a brightness threshold. An automatic thresholding procedure was carried out using the algorithm of Nobis and Hunziker (2005) after a gamma correction $(\Upsilon=2.2)$ (Moeser et al. 2014). The hemispherical photos were stratified into 5 rings with a step of $15^{\circ}$ (Moeser et al. 2014). Only the first two rings were used for canopy cover estimation, since Bunnell and Vales (1990) demonstrated that the canopy cover measurements were most accurate with view angles below $30^{\circ}$. Subsequently, the canopy cover was calculated as the ratio of canopy 
pixels to total pixels in the hemispherical photos (Table 4.1) (Moeser et al. 2014). LAI and average leaf angle (ALA) were also computed from the hemispherical photographs (Ali et al. 2016b).

Table 4.1 Summary of statistics regarding in situ measurements of leaf and canopy variables collected in Bavarian Forest National Park. (LMA: leaf dry mass per area, CD: crown diameter, SH: stand height, CC: canopy cover)

\begin{tabular}{lllllllll}
\hline & $\begin{array}{l}\text { LWC } \\
(\text { Needle } \\
\text { leaf }) \\
\left(\mathrm{g} / \mathrm{cm}^{2}\right)\end{array}$ & $\begin{array}{l}\text { LWC } \\
(\text { Broadleaf }) \\
\left(\mathrm{g} / \mathrm{cm}^{2}\right)\end{array}$ & $\begin{array}{l}\text { LWC } \\
(\text { Mixed }) \\
\left(\mathrm{g} / \mathrm{cm}^{2}\right)\end{array}$ & $\begin{array}{l}\text { LMA } \\
\left(\mathrm{g} / \mathrm{cm}^{2}\right)\end{array}$ & $\begin{array}{l}\text { LAI } \\
(\mathrm{m})\end{array}$ & $\begin{array}{l}\text { SH } \\
(\mathrm{m})\end{array}$ & $\begin{array}{l}\mathrm{CC} \\
(\%)\end{array}$ \\
\hline $\begin{array}{l}\text { Mean } \\
\text { Standard }\end{array}$ & 0.0260 & 0.0098 & 0.0184 & 0.0140 & 4.60 & 5.67 & 20.23 & 79.96 \\
deviation & 0.0025 & 0.0029 & 0.0034 & 0.0030 & 0.72 & 1.56 & 4.52 & 11.45 \\
Maximum & 0.0291 & 0.0135 & 0.0229 & 0.0291 & 6.21 & 10.55 & 27.36 & 95.24 \\
Minimum & 0.0215 & 0.0071 & 0.0135 & 0.0034 & 2.79 & 2.91 & 12.26 & 50.12 \\
\hline
\end{tabular}

\subsubsection{Airborne LiDAR and hyperspectral data collection}

An airborne LiDAR flight campaign was carried out at the end of July 2012 using a Riegl LMS-Q 680i sensor at a nominal height of $650 \mathrm{~m}$ above ground covering the whole park. The wavelength of the LiDAR data was $1550 \mathrm{~nm}$ and the data had an average point density of 30 points per $\mathrm{m}^{2}$. The system had a beam divergence of $0.5 \mathrm{mrad}$, a range accuracy of $20 \mathrm{~mm}$ and a maximum pulse repetition rate of 400,000 $\mathrm{Hz}$.

Airborne hyperspectral data were obtained with a HySpex sensor by German Aerospace Center (DLR) for the study area on July 22, 2013. The system comprises two imaging spectrometers with spectral ranges from 400 to $1000 \mathrm{~nm}$ across 160 channels (visible and near infrared, VNIR) and from 1000 to $2500 \mathrm{~nm}$ across 256 channels (short-wave infrared, SWIR). The spatial resolutions of these two spectrometers were $1.65 \mathrm{~m}$ and $3.3 \mathrm{~m}$, respectively. To coincide with the plot size, the data were resampled to $30 \mathrm{~m}$ resolution for the RTMs inversion. The data were collected at an average nominal altitude of 3000 $\mathrm{m}$ in 19 image strips. The data were preprocessed by DLR. Image data were converted from digital numbers to surface reflectance using the atmospheric correction model ATCOR4 based on atmospheric lookup tables generated with the radiative transfer model MODTRAN4 (Wang et al. 2016). Ortho-rectification was performed based on the parametric model/table using recorded attitude and flight path data in combination with a digital terrain model (DEM) (Wang et al. 2017).

\subsection{Methods}

Firstly, LiDAR intensity was investigated for LWC estimation. Secondly, prior knowledge obtained from the combination of the two sensors was evaluated when coupled in RTMs. The prior knowledge consisted of soil reflectance, canopy cover and 
understory LAI. Soil reflectance was obtained from the combination of LiDAR and hyperspectral data, and a range of spectra were used as input in the forward mode of RTMs to simulate canopy reflectance. Canopy cover and understory LAI were directly estimated from LiDAR data, and then used to constrain the RTM inversion

\subsubsection{Leaf water content estimation from LiDAR intensity}

The LiDAR intensity was mainly affected by the range, incidence angle and atmospheric conditions (Höfle and Pfeifer 2007). The atmospheric conditions were assumed to be constant during the flight campaign (Fieber et al. 2013). The correction of range and incidence angle effects was based on the radar equation (Höfle and Pfeifer 2007):

$$
I_{r}=I \frac{R^{2}}{R_{S}^{2}} \frac{1}{\cos \alpha}
$$

where $I_{r}$ is the corrected intensity, $I$ is recorded intensity, $R$ is the range between the sensor and the target, $\alpha$ is the angle of incidence between the laser beam and surface normal, and $R_{s}$ is the normalized range. Single returns were used for LWC estimation, as they were less affected by the area of collision between the laser beam and the target.

To calculate the range $R$ between the sensor and the target, the GPS location of the sensor was interpolated using the recorded GPS time of each return, since the frequency of the GPS recording of the sensor was lower than that of the laser beams. Then the range was calculated between the location of the sensor and the target.

The incidence angle is the angle between the surface normal of each plot and the laser beam. The surface normal was estimated based on the analysis of the eigenvectors of the covariance matrix of the point cloud (Zhu et al. 2015). The vector of the laser beam was obtained based on the point location and the sensor location.

\subsubsection{Retrieval of soil reflectance}

LiDAR point clouds were classified to extract ground returns and non-ground returns using the Lasground module in Lastools (Lastools, rapidlasso GmbH, Germany, 2017) to produce the digital elevation model (DEM). All LiDAR points were then normalized by subtracting the DEM. After normalization, the elevation value of a point indicated the height from the ground to that point. The canopy height model (CHM) matching the resolution of the hyperspectral data $(3.3 \mathrm{~m})$ was created from the LiDAR point cloud using a spike-free algorithm (Khosravipour et al. 2016). The co-aligned LiDAR and hyperspectral data were used to extract the soil reflectance of the study site. For this, 10 large areas (22648 pixels in total) in the southern part of the park were randomly selected. Within these areas, we firstly applied a dilation with a $3 * 3$ moving window to the CHM to enlarge the tree crowns and reduce the effect of shadow. The value of the center pixel was updated by the maximum value of all pixels in the $3 * 3$ moving window (MATLAB, The MathWorks Inc., USA, 2016). To capture the soil reflectance, pixels in the hyperspectral images with a height below $0.1 \mathrm{~m}$ in the CHM were considered to be soil 
pixels. In total, 3497 soil pixels were thus obtained. To parameterize the RTM, the maximum and minimum reflectance of the soil were selected from the 3497 soil pixels. A scale factor was randomly generated to allow the soil reflectance varying within this range.

\subsubsection{Canopy cover estimation}

LiDAR point clouds were classified to extract ground returns and non-ground returns using the Lasground module in Lastools (Lastools, rapidlasso GmbH, Germany, 2017) to produce the digital elevation model (DEM). All LiDAR points were then normalized by subtracting the DEM. After normalization, the elevation value of a point indicated the height from the ground to that point. A threshold of $1.3 \mathrm{~m}$ was used to separate canopy returns and below-canopy returns to coincide with the height of the hemispherical photographs (Korhonen et al. 2011). A Beer's Law modified (two-way transmission loss) intensity ratio (IR) (Hopkinson and Chasmer 2009) was used to estimate canopy cover in the Matlab software (MATLAB, The MathWorks Inc., USA, 2016) (Eq. (4.4)).

$$
I R=1-\left(\frac{\left(\frac{\sum I_{\text {GroundSingle }}}{\sum I_{\text {Total }}}\right)+\sqrt{\frac{\sum I_{\text {GroundLast }}}{\sum I_{\text {Total }}}}}{\left(\frac{\sum I_{\text {First }}+\sum I_{\text {Single }}}{\sum I_{\text {Total }}}\right)+\sqrt{\frac{\sum I_{\text {Intermediate }}+\sum I_{\text {Last }}}{\sum I_{\text {Total }}}}}\right)
$$

where $I$ represents the intensity, and each subscript refers to the class of pulse return.

\subsubsection{Understory leaf area index}

A threshold of $1.3 \mathrm{~m}$ was applied to remove canopy returns. Eq. (4) was used to calculate understory cover. Understory LAI was then calculated based on (Chen and Cihlar 1995):

$$
L A I_{u}=\left(-\ln \left(1-C C_{u}\right)\right) \cos \theta / G
$$

where $\mathrm{CC}_{\mathrm{u}}$ is the understory cover, $\theta$ is the LiDAR viewing zenith angle, $G$ is the fraction of the leaf area projected on a plane normal to the zenith angle $\theta$. $G$ is calculated as ( $\mathrm{Li} \mathrm{et}$ al. 2017):

$$
\mathrm{G}=\frac{\left(x^{2}+\tan ^{2} \theta\right)^{0.5} \cos \theta}{x+1.774(x+1.182)^{-0.733}}
$$

where $x$ is a shape parameter of the leaf angle distribution. A spherical distribution with $x=1$ was applied in this study ( $\mathrm{Li}$ et al. 2017).

\subsubsection{Radiative transfer model}

The invertible forest reflectance model (INFORM) (Atzberger 2000) was chosen for canopy reflectance modeling and inversion. The choice of INFORM was a trade-off between suitability for forest structure characterization and simplicity in generating scenes and inversion (Schlerf and Atzberger 2012). The model is an combination of the 
forest light interaction model (FLIM) (Rosema et al. 1992), SAILH (Verhoef 1985) and PROSPECT (Jacquemoud and Baret 1990), simulating the bidirectional reflectance of forest stands between 400 and $2500 \mathrm{~nm}$. INFORM is hybrid model with geometricaloptical (crown shadowing) and turbid-medium (multiple scattering) representation (Schlerf and Atzberger 2006). Our model adopts the same parameterization values as developed by (Ali et al. 2017) to estimate specific leaf area.

\subsubsection{INFORM parameterization and generation of look-up table}

At leaf level, the leaf structure parameter $(\mathrm{N})$ was fixed at 1.5 since it has little influence on canopy reflectance (Ali et al. 2016b; Jacquemoud 1993). The chlorophyll content was arbitrarily set from 40 to $80\left(\mu \mathrm{g} / \mathrm{cm}^{2}\right)$ (Ali et al. 2016b; Ali et al. 2016c). For the other leaf parameters such as LWC $\left(\mathrm{g} / \mathrm{cm}^{2}\right)$ and leaf dry mass $\left(\mathrm{g} / \mathrm{cm}^{2}\right)$, the range was selected based on the range of the variables as measured in the field.

The model input canopy structural parameters consisted of stem density (SD, n/ha), stand height $(\mathrm{SH}, \mathrm{m})$, crown diameter $(\mathrm{CD}, \mathrm{m})$ and ALA (degree); of which $\mathrm{SD}$ and $\mathrm{CD}$ were mainly used to calculate ground covered by crowns $\left(\mathrm{C}_{\mathrm{o}}\right)$ and by shadow $\left(\mathrm{C}_{\mathrm{s}}\right)$ under an observation zenith angle $\theta_{\mathrm{o}}$ (degree) and a solar zenith angle $\theta_{\mathrm{s}}$ (degree), respectively, using Eq. (4.7) (Atzberger 2000):

$$
C=1-\exp (-\mathrm{k} \cdot \mathrm{SD} / \cos (\theta))
$$

where $\mathrm{k}$ represents the average horizontal area of a single tree crown (ha).

When observed at nadir, ground covered by crowns $\left(\mathrm{C}_{\mathrm{o}}\right)$ equals canopy cover $(\mathrm{CC})$ : the proportion of the forest floor covered by the vertical projection of the tree crowns (Jennings et al. 1999). To obtain SD and CD estimations, segmentation must be performed on individual trees. Recent studies have shown that although canopy height can be retrieved from LiDAR with high accuracy, the retrieval of SD and CD from LiDAR segmentation is complicated and introduces uncertainty (Morsdorf et al. 2004; Reitberger et al. 2009). On the other hand, canopy cover can be retrieved from LiDAR with much less uncertainty (Hopkinson and Chasmer 2009; Korhonen et al. 2011; Moeser et al. 2014). In addition, SD and CD are used to calculate canopy cover which is main input to simulate reflectance in INFORM. Therefore, in this study, SD and CD were replaced with canopy cover in the INFORM forward mode for the simulation of canopy reflectance. The range of forest canopy cover was set from 0.1 (canopy cover above 10 $\%$ ) to 1 (full canopy cover) according to the definition of forest provided by the Forest and Agriculture Organization of the United Nations' definition of forests (FAO 2017). The ranges of other structural parameters (i.e. single tree LAI, LAI, understory LAI, SH, $\mathrm{CD}$, and ALA (degree)) were set based on prior information obtained from field observations. The sun zenith angle (degree), observation angle (degree) and azimuth angle (degree) were determined based on the HySpex image acquisition campaign (Table 
4.2). The standard soil reflectance for comparison purpose was generated using wet and dry soil spectra (Feret et al. 2008), a scale factor was randomly generated to allow the soil reflectance to vary between these two spectra. The soil reflectance derived from hyperspectral and LiDAR data was described in $\S 4.3 .2$. In total, 100,000 combinations were randomly generated and the simulated reflectances were stored in LUT.

Table 4.2 The input parameters and their ranges for LUT generation using INFORM

\begin{tabular}{lllll}
\hline Parameter & Abbreviation & Unit & Minimum value & Maximum value \\
\hline Leaf dry mass & $\mathrm{C}_{\mathrm{m}}$ & $\mathrm{g} / \mathrm{cm}^{2}$ & 0.005 & 0.03 \\
Leaf water content & $\mathrm{LWC}$ & $\mathrm{g} / \mathrm{cm}^{2}$ & 0.005 & 0.035 \\
Leaf structural parameter & $\mathrm{N}$ & - & 1.5 & 1.5 \\
Chlorophyll content & $\mathrm{C}_{\mathrm{ab}}$ & $\mu \mathrm{g} / \mathrm{cm}^{2}$ & 40 & 40 \\
Single tree LAI & $\mathrm{LAI}_{\mathrm{s}}$ & - & 2 & 8 \\
Understory LAI & $\mathrm{LAI}_{\mathrm{u}}$ & - & 0 & 1 \\
Canopy cover & $\mathrm{CC}$ & - & 0.1 & 1 \\
Stand height & $\mathrm{SH}$ & $\mathrm{m}$ & 5 & 40 \\
Crown diameter & $\mathrm{CD}$ & $\mathrm{m}$ & 2 & 10 \\
Average leaf angle & $\mathrm{ALA}$ & degree & 40 & 60 \\
Sun zenith angle & $\theta_{\mathrm{s}}$ & degree & 28 & 38 \\
Observation zenith angle & $\theta_{\mathrm{o}}$ & degree & 0 & 0 \\
Azimuth angle & $\Psi$ & degree & 126 & 182 \\
Fraction of diffused radiation & $\mathrm{Skyl}$ & - & 0.1 & 0.1 \\
\hline
\end{tabular}

\subsubsection{The look-up table inversion}

The simplest and most popular method to solve the inverse problem for RTMs is to use minimization algorithms through LUTs (Combal et al. 2003; Darvishzadeh et al. 2008b). The inversion problem is based on the minimization of a cost function $\delta^{2}$ that concurrently measures the discrepancies between 1) the observed and simulated reflectance and 2) variables to estimate and the associated prior information (Jacquemoud et al. 2009):

$$
\delta^{2}=\sum_{i=1}^{n}\left(\frac{\rho_{o}-\rho_{s}}{\sigma\left(\rho_{o}\right)}\right)^{2}+\sum_{j=1}^{k}\left(\frac{\chi_{j}-\chi_{j}^{\text {prior }}}{\sigma\left(\chi_{j}\right)}\right)^{2}
$$

where $\rho_{o}$ and $\rho_{s}$ are the observed and simulated reflectance, respectively; $\chi_{\mathrm{j}}$ and $\chi^{\text {prior }}{ }_{j}$ are the estimated values of the simultaneously retrieved variables and the prior values, respectively; and $\sigma$ indicates the variance. In this case, the prior information of canopy cover and understory LAI was measured from LiDAR.

\subsubsection{Statistical analysis}

The estimated variables were tested against the measured variables based on the coefficient of determination $\left(R^{2}\right)$, the root mean square error (RMSE), normalized RMSE, as well as normalized bias. In order to compare the estimated and measured variables, the 
regression line was forced to the origin $(y=x)$, and the $R^{2}$ was calculated accordingly. Leave-one-out cross validation was used for the validation of LWC estimation using LiDAR intensity data.

$$
\begin{gathered}
R^{2}=1-\frac{\sum\left(y_{i}-y_{i}^{\prime}\right)^{2}}{\sum\left(y_{i}-\overline{y_{i}}\right)^{2}} \\
\text { RMSE }=\sqrt{\frac{\sum\left(y_{i}-y_{i}^{\prime}\right)^{2}}{n}} \\
\text { nRMSE }=R M S E / \overline{y_{i}} \\
\text { nBias }=\frac{\sum\left(y_{i}-y_{i}^{\prime}\right)}{n \overline{y_{i}}}
\end{gathered}
$$

where $y_{i}$ and $y_{i}^{\prime}$ are the measured and estimated values for sample $i$, and $\overline{y_{i}}$ and $n$ are the mean and the number of samples, respectively.

\subsection{Results}

\subsubsection{Leaf water content estimation from LiDAR intensity}

Fig. 4.2 shows that corrected LiDAR intensity produced a moderate accuracy for LWC estimation with an $\mathrm{R}^{2}$ value of 0.59 and nRMSE of 0.26 .

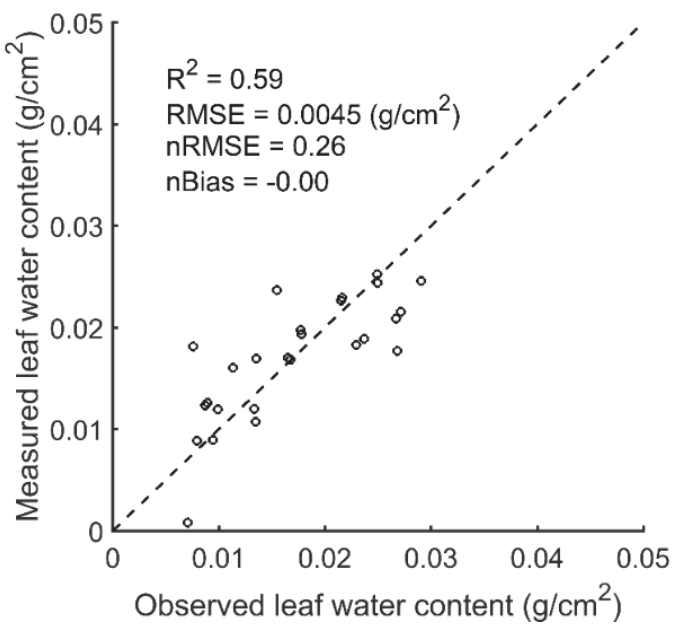

Figure 4.2 Cross validation of LWC estimation using LiDAR intensity 


\subsubsection{Background reflectance}

The background pixels were initially derived from hyperspectral images, being pixels for which the corresponding height in the original CHM was less than $0.1 \mathrm{~m}$ (Fig. 4.3(a) and (c)). Fig. 4.3(b) shows the CHM after $3 * 3$ dilation to avoid shadow on the background pixels. As the tree crowns became larger, fewer pixels (red dots) were selected from the $\mathrm{CHM}$ after the dilation.

Fig. 4.4 shows the mean and spectral variability of background reflectance from the spectral imagery and LiDAR CHM, and the standard soil reflectance (dotted line). For the derived background reflectance using LiDAR height information, the maximum and the mean showed vegetation characteristics with a small peak in the NIR region except for the minimum spectrum, which represents soil without chlorophyll. This indicated that the background was not only composed of soil, but also of low vegetation such as short grass and moss.
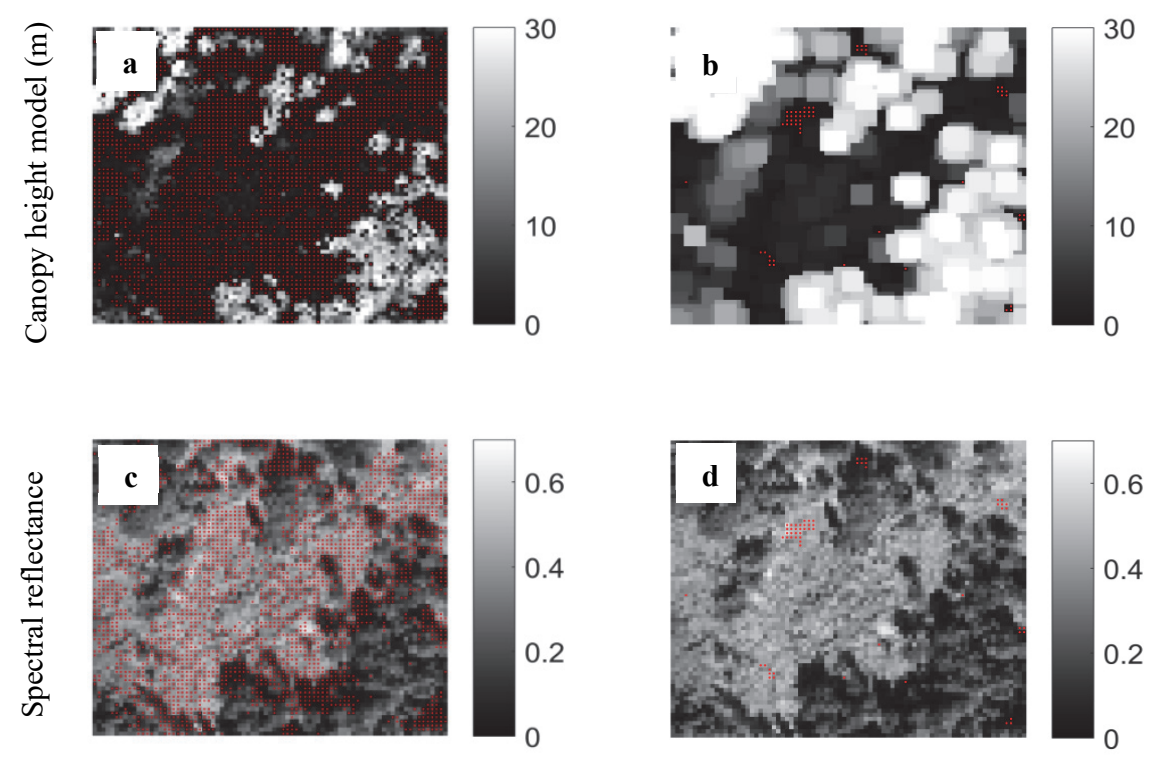

Figure 4.3 An example of background pixels from hyperspectral image and canopy height model. (a) original canopy height model (b) dilated canopy height model; (c) and (d) spectral reflectance (red pixels indicate height below $0.1 \mathrm{~m}$ ), before and after dilation, respectively 


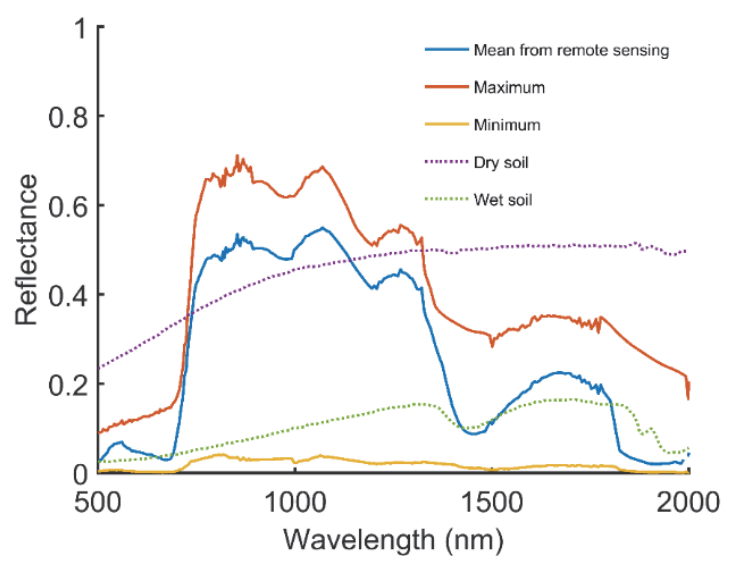

Figure 4.4 Mean and spectral variability of the soil reflectance spectrum (dotted line: standard soil; solid lines: from remote sensing derived background)

\subsubsection{Canopy cover and understory leaf area index}

The LiDAR-derived canopy cover was compared with the canopy cover estimated from the hemispherical photos for the 26 forest plots (Fig. 4.5). The scatterplot showed a good correlation with an $\mathrm{R}^{2}$ value of 0.63 . The low nRMSE value $(0.08)$ indicates that the LiDAR index is suitable for canopy cover estimation.

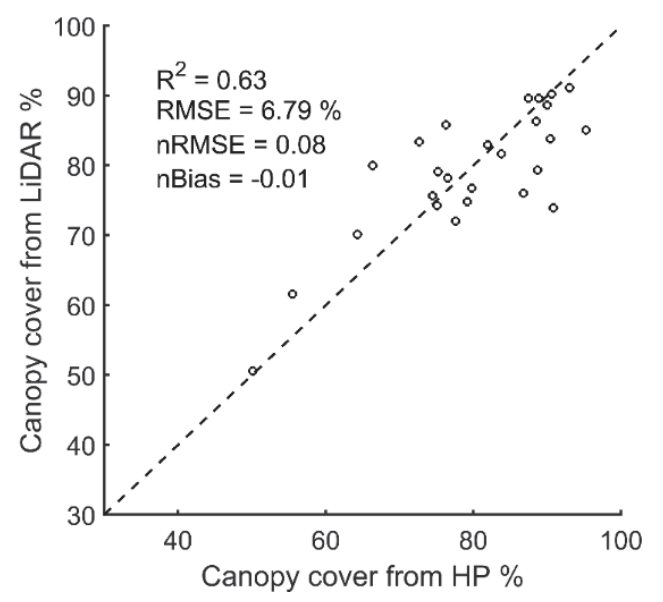

Figure 4.5 Scatterplot showing the relationship between canopy cover obtained from hemispherical photos and the LiDAR index

\subsubsection{Retrieval of leaf water content by inversion}

The roles of LiDAR-derived canopy cover and background reflectance used as prior information in the model inversion for the retrieval of LWC were evaluated on the basis 
of $R^{2}$, RMSE, nRMSE and nBias values. Fig. 4.6(a) demonstrates that the inversion of INFORM for the retrieval of LWC using hyperspectral data alone (without prior information) yielded a low $R^{2}$ with a large bias. After introducing prior information of remote sensing derived soil reflectance in the model, the inversion accuracy significantly increased $\left(R^{2}=0.72\right)$ and the error decreased (nRMSE $\left.=0.22\right)$ (Fig. 4.6(b)), while the introduction of prior information of understory LAI and canopy cover further improved the accuracy marginally.
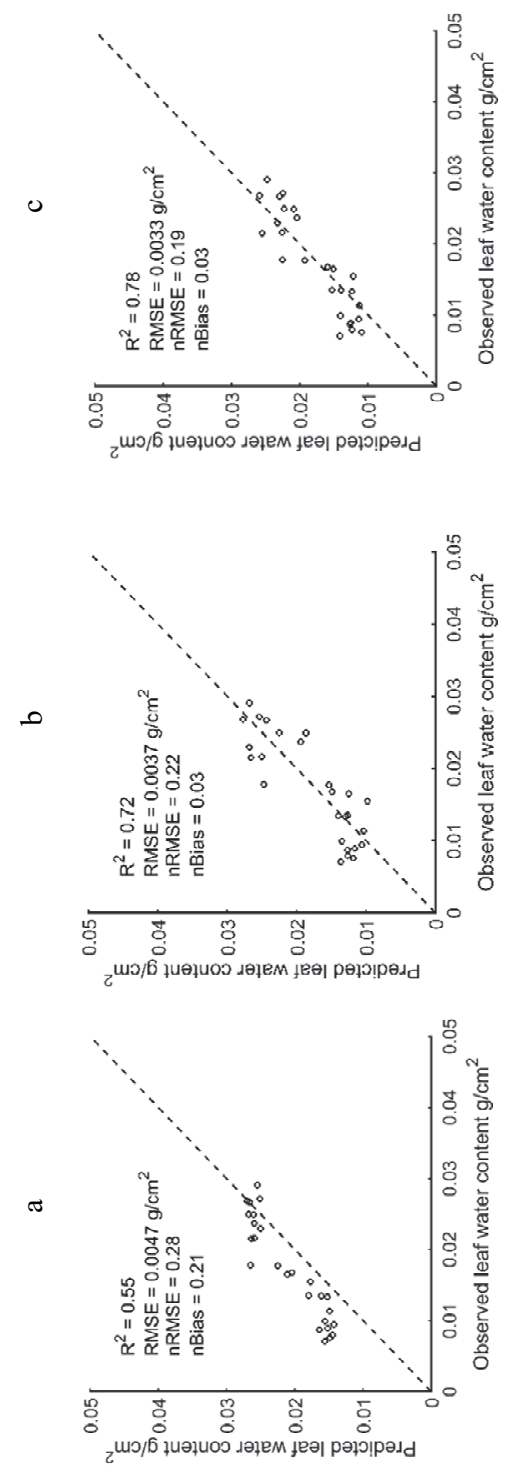

Figure 4.6 Scatterplots between observed leaf water content and predicted leaf water content obtained from (a) inversion of LUT using hyperspectral only; (b) using prior information of remote sensing soil reflectance; (c) using all prior information 


\subsubsection{Mapping of leaf water content using LiDAR and hyperspectral data}

The HySpex image was masked to extract the forest areas from the land cover map provided by the Bavarian Forest National Park. The image and the aligned LiDAR data were used as input in the model inversion of INFORM to produce the map of LWC (Fig. 4.7). In addition, LWC of broadleaf, needle leaf and mixed forests with different stand ages was also extracted using the land cover map. On average, LWC was higher in the areas with lower latitude where needle forests were located.

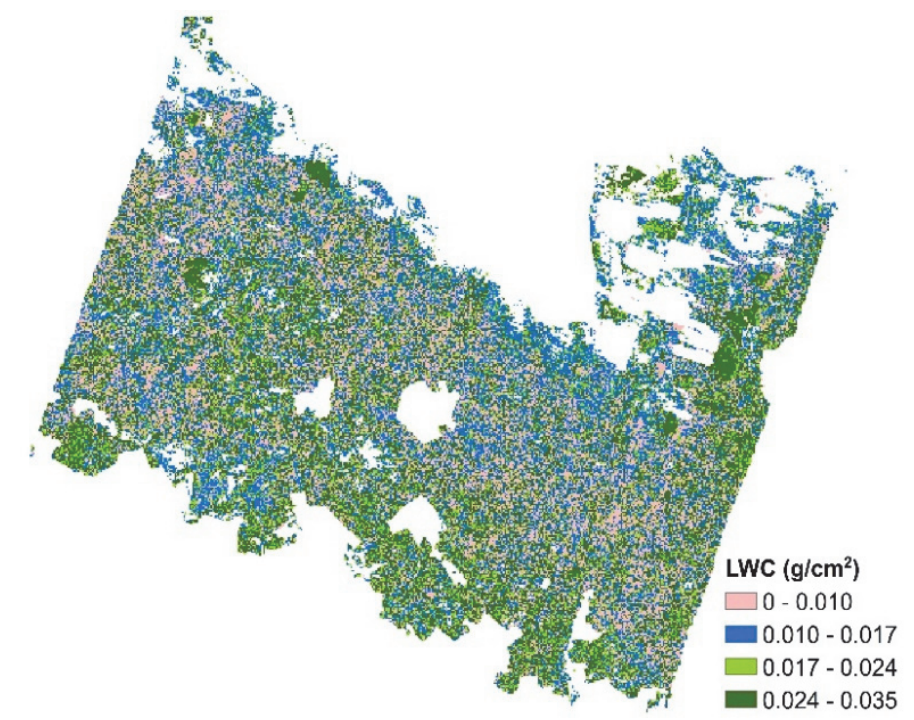

Figure 4.7 Leaf water content mapping of Bavarian Forest National Park using LiDAR and hyperspectral data

We compared the LWC map with the forest type map. The spatial variation of LWC was in accordance with the distribution of broadleaf, needle leaf and mixed forests (Table 4.3). Needle leaf forests had higher LWC than mixed and broadleaf forests. For the needle leaf forests, the LWC was higher in mature forests, while for broadleaf forests, younger forests had higher LWC. 
Retrieval of leaf water content from airborne LiDAR and hyperspectral data

Table 4.3 Summary of statistics for leaf water content of different forest types

\begin{tabular}{|c|c|c|c|c|c|c|c|c|c|}
\hline \multirow[t]{2}{*}{ Forest type } & \multicolumn{3}{|c|}{ Needle leaf } & \multicolumn{3}{|c|}{ Broadleaf } & \multicolumn{3}{|l|}{ Mixed } \\
\hline & Mature & Medium & Young & Mature & Medium & Young & Mature & Medium & Young \\
\hline $\begin{array}{l}\text { Mean leaf } \\
\text { water content } \\
\left(\mathrm{g} / \mathrm{cm}^{2}\right)\end{array}$ & 0.022 & 0.021 & 0.017 & 0.015 & 0.017 & 0.018 & 0.019 & 0.019 & 0.018 \\
\hline $\begin{array}{l}\text { Standard } \\
\text { deviation } \\
\left(\mathrm{g} / \mathrm{cm}^{2}\right)\end{array}$ & 0.008 & 0.009 & 0.007 & 0.009 & 0.009 & 0.008 & 0.010 & 0.009 & 0.008 \\
\hline $\begin{array}{l}\text { Mean of all } \\
\left(\mathrm{g} / \mathrm{cm}^{2}\right)\end{array}$ & 0.021 & & & 0.016 & & & 0.019 & & \\
\hline
\end{tabular}

\subsection{Discussion and conclusions}

We estimated leaf water content (LWC) in the Bavarian Forest National Park from airborne LiDAR and hyperspectral data. Firstly, LiDAR intensity was investigated for the estimation of LWC by correcting the range and incidence angle effects. Secondly, the prior information obtained from the combination of LiDAR and hyperspectral data was introduced as an input in the modelling and inversion of RTMs for LWC estimation. Results show that $\mathrm{LWC}$ was estimated using LiDAR intensity alone with an $\mathrm{R}^{2}$ value of 0.59 and nRMSE value of 0.26 . When combining the information from the two systems in the RTMs inversion, a significantly (t-test; $p<0.05$ ) higher retrieval accuracy of LWC with an $\mathrm{R}^{2}$ value of 0.78 and an nRMSE of 0.19 was obtained than with the results derived from hyperspectral data alone $\left(\mathrm{R}^{2}=0.55, \mathrm{nRMSE}=0.28\right)$

The results show that the introduction of the soil reflectance derived from remote sensing significantly improved the accuracy of LWC estimation. In this study, the soil reflectance was derived from hyperspectral images with a CHM height filter. The height threshold $(0.1 \mathrm{~m})$ minimized the influence of the forest understory layer, which needs to be separated from the soil reflectance in INFORM modeling. The standard soil reflectance, did not appear to represent background variation as well as the remote sensing derived soil reflectance, the product of a larger collection of samples. The wide range of soil reflectance derived from remote sensing indicated that the background in the park was not only composed of soil, but also vegetation such as grass, moss, lichen and plant litter. Allowing the soil reflectance to change within the range of remote sensing derived soil spectra in the LUT generation could better map the variation of the real background than using a standard (single) soil reflectance.

The introduction of canopy cover and understory LAI improved the RMSE of LWC estimation by $13.6 \%$. The mean canopy cover of our plots was $79.96 \%$ with a minimum value of $50.12 \%$. In closed canopies, canopy reflectance is less affected by canopy structure (Xiao et al. 2014). Forest traits can be accurately estimated from canopy reflectance, irrespective of the heterogeneity of structural variables, providing that canopy 
cover exceeds $50 \%$ (Ali et al. 2016b). In addition, due to the high canopy cover, the understory LAI was relatively low (0-1.1). The limited range of understory LAI did not have a large impact on the variation of canopy reflectance. Nevertheless, the prior information of canopy cover and understory LAI was important to minimize the impact of canopy structure and background on canopy reflectance. This technique would work better in a more heterogeneous forest-grassland system, where the variation of canopy cover is larger (e.g. $10 \%-100 \%)$.

In order to fully explore the potential of LiDAR in canopy structure characterization, we employed canopy cover to replace the two structural parameters, stem density (SD) and crown diameter (CD), in INFORM. Our results show that canopy cover can be retrieved using LiDAR accurately (nRMSE $=0.08$ ). We also tried to detect single trees with a segmentation method (i.e. watershed) in our study area, but the accuracy of detection rate was low (nRMSE $>0.45$, not shown in this paper). We expect the influence of replacing $\mathrm{SD}$ and $\mathrm{CD}$ with canopy cover on the inversion results to be minimal. In INFORM, SD and $\mathrm{CD}$ are used to calculate canopy cover for reflectance simulation, so the only discrepancy this modification may have caused is a difference in range. If the range of $\mathrm{SD}$ and $\mathrm{CD}$ was to be converted from the range of canopy cover using the INFORM formula, the only difference in the generated LUTs would be caused by randomness in the LUT generation.

LiDAR systems do not only deliver geometric information, but also radiometric information such as backscatter intensity. Previous studies have shown the potential of airborne LiDAR data for object classification (Mallet et al. 2011; Zhang and Liu 2012). This study demonstrates that airborne LiDAR intensity data could be potentially used for the estimation of LWC. However, there are a number of challenges when using airborne intensity to estimate essential biodiversity variables (Zhu et al. 2015). The incidence angle was calculated based the angle between the laser beam and the surface plane of a plot, and then the incidence angle effects were corrected based on the radar equation. However, forests, especially needle leaf forests, are highly anisotropic. The incidence angle effects are more complicated than when dealing with homogeneous areas such as road and grassland (Kim et al. 2009). In addition, even within the crown of a single tree, the topography changes. Initially in this study, the surface plane was constructed based on a radius of $1 \mathrm{~m}$ to model the local incidence angle within a crown, but the cross validation results indicated that correction of the incidence angle was not effective (not shown in this paper). This issue could potentially be overcome by using a LiDAR system with multiple wavelengths. By using a ratio of two bands which are similarly affected by the incidence angle, the effects could be canceled out as proven on a TLS system by (Gaulton et al. 2013; Hancock et al. 2017).

The map of LWC in the Bavarian Forest National Park showed a spatial variation in accordance with the forest types map provided by the Bavarian Forest National Park administration. Needle leaf forests which are mostly located at low latitude had a higher 
LWC than broadleaf forests at high latitude, which was also observed in our laboratory measurements (Table 4.1). This phenomenon also follows Hatcher (1990) observation that young broadleaf trees have a higher LWC than mature ones. A recent study (Chavana - Bryant et al. 2016) demonstrated monotonic decreases in LWC with age across broadleaf trees. For needle leaf trees, however, our mapping showed that mature trees had a higher LWC than young ones (Table 4.3)

The LiDAR-derived canopy cover was compared with that from hemispherical photos. Although the scan zenith angles mostly fell within $0-30^{\circ}$, they varied between plots. The viewing angles of hemispherical photos were more consistent. This could be one of the reasons causing the discrepancy between the results derived from these two techniques. Even though an automatic thresholding method was applied, changing sun angle and weather conditions during the field work may also cause inconsistency.

There were some noteworthy temporal differences between the field measurements and the remote sensing data. In particular, LiDAR data were obtained in July 2012, while the field data were measured in July and August 2013. We expect the change in the forest canopy structure to be minimal since the data were both collected in the summer when the leaves had stopped growing. The hyperspectral data were collected in July 2013, which is in the same year the fieldwork took place. However, some plots were measured in August, and LWC could have changed due to different weather conditions. More accurate results may have been obtained had all plots been measured at the same time as the hyperspectral data. 


\section{Chapter 5}

\section{Foliar and woody materials discriminated using terrestrial LiDAR in a mixed natural forest*}

* Zhu, X., Skidmore, A.K., Darvishzadeh, R., Niemann, K.O., Liu, J., Shi, Y., \& Wang, T. (2018). Foliar and woody materials discriminated using terrestrial LiDAR in a mixed natural forest. International Journal of Applied Earth Observation and Geoinformation, 64, 43-50. 


\begin{abstract}
Separation of foliar and woody materials using remotely sensed data is crucial for the accurate estimation of leaf area index (LAI) and woody biomass across forest stands. In this paper, we present a new method to accurately separate foliar and woody materials using terrestrial LiDAR point clouds obtained from ten test sites in a mixed forest in Bavarian Forest National Park, Germany. Firstly, we applied and compared an adaptive radius near-neighbor search algorithm with a fixed radius near-neighbor search method in order to obtain both radiometric and geometric features derived from terrestrial LiDAR point clouds. Secondly, we used a random forest machine learning algorithm to classify foliar and woody materials and examined the impact of understory and slope on the classification accuracy. An average overall accuracy of $84.4 \%(\mathrm{Kappa}=0.75)$ was achieved across all experimental plots. The adaptive radius near-neighbor search method outperformed the fixed radius near-neighbor search method. The classification accuracy was significantly higher when the combination of both radiometric and geometric features was utilized. The analysis showed that increasing slope and understory coverage had a significant negative effect on the overall classification accuracy. Our results suggest that the utilization of the adaptive radius near-neighbor search method coupling both radiometric and geometric features has the potential to accurately discriminate foliar and woody materials from terrestrial LiDAR data in a mixed natural forest.
\end{abstract}




\subsection{Introduction}

Forest structure can have profound effects on ecosystem function and diversity, including mass and energy cycling through transpiration and photosynthesis (Hosoi et al. 2013; Spies 1998). In order to understand and manage forest structure, many forest structural parameters have been developed using various remote sensing techniques, such as leaf area index (LAI), biomass and height (Ali et al. 2016a; Hosoi et al. 2010; Lucas et al. 2008). LAI is one of most used and essential forest structural parameters. The estimation of LAI from remote sensing requires the accurate separation between foliage and woody materials. Passive optical remote sensing has been widely used to estimate forest structural variables and essential biodiversity variables (Schlerf and Atzberger 2006; Skidmore et al. 2015; Treitz and Howarth 1999). However, mapping and monitoring forest structure have been challenging with existing passive optical sensors, as in 2D image pixels, different objects are often mixed or overlapped (Wagner et al. 2008a).

Light detection and ranging (LiDAR) provides an advantage over passive remote sensing in its ability to capture 3D information about forest structure (Cao et al. 2016; Henning and Radtke 2006). In particular, terrestrial laser scanning (TLS) data with very high point density and small beam size, would allow an accurate characterization of forest structure (Hosoi et al. 2010). Moreover, using high point density data, TLS is able to capture the geometric features of different vegetation elements and ground (Zheng et al. 2016a). Clawges et al. (2007) estimated leaf area by subtracting the leaf-off returns from leaf-on returns. But this approach requires both leaf-off and leaf-on returns, which is not practical for evergreen vegetation such as coniferous forests. Ma et al. (2016b) applied a geometricbased classification algorithm to separate photosynthetic and nonphotosynthetic components using TLS data, indicating that the local dimensionality features (how the point clouds are geometrically distributed) played a significant role in differentiating leaves and stems as leaves had a random feature and stems had a linear feature. In addition to geometric information, radiometric information from TLS data has also been applied to object detection and forest parameter retrieval (Penasa et al. 2014; Zhu et al. 2017). Zhao et al. (2011) used a threshold on the ratio of total power to reflected pulse width to separate foliage and woody materials, since woods were solid targets that produced a sharply peaked return pulse compared to foliage material. Danson et al. (2014) suggested that the Salford Advanced Laser Canopy Analyser (SALCA), a dual-wavelength LiDAR could be used to derive a ratio of 'apparent reflectance' that was insensitive to laser incidence angle. Thus, it allowed the classification of different structural components of vegetation. Although radiometric and geometric features derived from TLS data have been used separately, their combination for separation of foliar and woody materials needs to be further explored.

In order to obtain the geometric and radiometric features of a point, a local neighborhood point set is needed. The local neighborhood is defined as a spherical shape (Demantke et 
al. 2011), where local points are within a fixed radius (Koenig et al. 2015). The local point set within a spherical neighborhood can be used to calculate the local dimensionality that indicates whether the points are more likely to be a line, a plane surface, or a random cluster (Brodu and Lague 2012). However, the size of the radius used to search for neighborhood points affects the geometric dimensionality (Yang and Dong 2013) and radiometric features (Koenig et al. 2015), which can result in misclassification. As demonstrated by Brodu and Lague (2012), at a few centimeter scale, leaves have a plane surface dimensionality, while at a larger scale (i.e. $30 \mathrm{~cm}$ ), they have a random dimensionality (3D). Ma et al. (2016b) conducted a sensitivity analysis on the effects of the searching radius on photosynthetic and nonphotosynthetic components classification, suggesting that $0.45 \mathrm{~m}$ would be a good choice for a single TLS location-based forest plot with mid-successional ages. In another study, Ma et al. (2016a) suggested that the diameter at breast height (DBH) is a good reference to choose an appropriate size of the searching radius. Koenig et al. (2015) calculated the local neighborhood features with four radius thresholds in crop fields. This multi-scale approach can be used in forest plots with heterogeneous DBHs so that the selection of a specific radius is not needed. However, it could result in very high dimensional features and low computational efficiency in a forest plot due to a large amount of features it produces. An adaptive radius near-neighbor search method (Demantke et al. 2011) choosing the optimal radius might be a good choice for the classification between foliar and woody materials.

The classification accuracy is usually compromised by other factors. Previous studies have shown that the understory and slope have a strong influence on the accuracy of LiDAR-derived forest parameters (e.g. canopy height, fractional cover) (Spaete et al. 2011; Su and Bork 2006; Wasser et al. 2010). Thus, in order to assess the classification results, we also evaluate the influence of understory and slope on the classification accuracy.

This study aims to classify foliar and woody materials using TLS data in a mixed natural forest. Specifically, we address the following research questions: (1) Does the combination of geometric and radiometric features significantly improve the classification of foliar and woody materials using TLS data results compared to those of using either one of them alone? (2) How is the performance of the adaptive radius nearneighbor search method in comparison to the commonly used fixed radius near-neighbor search method for the classification of foliar and woody materials using TLS data? and (3) Do understory and slope significantly affect the classification accuracy of foliar and woody materials using TLS data? 


\subsection{Study area and data}

\subsubsection{Study area}

The study was carried out in Bavarian Forest National Park in south eastern Germany. The natural forest ecosystems of the Bavarian Forest National Park vary according to altitude: there are alluvial spruce forests in the valleys, mixed mountain forests on the hillsides and mountain spruce forests in the high areas. Data from the TLS and in situ measurements were acquired in summer 2016 at four broadleaf, two coniferous, and four mixed circular plots of $10 \mathrm{~m}$ radius with varying density and height. In each plot, we recorded tree species, acquired LiDAR data with the TLS and took hemispherical photos in order to compare the results with LiDAR. Hemispherical photos were processed using the 'Hemisfer' software (Hemisfer, Swiss Federal Institute for Forest, Snow and Landscape Research WSL, Switzerland, 2016) to calculate the plant area index. Hemispherical photos were classified into sky and canopy pixels by applying a brightness threshold. An automatic thresholding procedure was carried out using the algorithm of Nobis and Hunziker (2005) after a gamma correction $(\Upsilon=2.2)$ (Moeser et al. 2014). The description of the data collected in each plot is given in Table 5.1.

Table 5.1 Plot characteristics collected during the fieldwork in Bavarian Forest National Park, Germany.

\begin{tabular}{cccc}
\hline Plot & Vegetation type & Dominant tree species & $\begin{array}{c}\text { Effective leaf area } \\
\text { index }\end{array}$ \\
\hline 1 & Broadleaf & Beech & 2.83 \\
2 & Conifer & Spruce & 0.91 \\
3 & Mixed & Beech, Spruce & 3.08 \\
4 & Conifer & Spruce & 3.18 \\
5 & Broadleaf & Beech & 3.46 \\
6 & Mixed & Beech, Spruce, Fir & 3.14 \\
7 & Mixed & Beech, Spruce & 3.10 \\
8 & Mixed & Beech, Spruce & 3.44 \\
9 & Broadleaf & Beech & 3.17 \\
10 & Broadleaf & Beech & 3.68 \\
\hline
\end{tabular}

\subsubsection{Terrestrial laser scanning data}

The TLS used in this study was a time-of-flight scanner RIEGL VZ-400 (Riegl LMS $\mathrm{GmbH}$, Horn, Austria). It employs a shortwave infrared $(1550 \mathrm{~nm})$ laser. The laser beam is $7 \mathrm{~mm}$ in diameter as it leaves the device. The system has a beam divergence of 0.35 mrad, a range accuracy of $5 \mathrm{~mm}$ and an effective measurement rate of 122,000 measurements/second. The pulse energy follows a Gaussian distribution within the laser beam. The data were acquired in a long-range mode and an angular step of $0.04^{\circ}$. To cover the whole plot, a center scan and three triangular scan positions were used for each plot (Béland et al. 2011). Data were registered with RISCAN Pro software (RIEGL Laser Measurement Systems, 2016). 


\subsection{Method}

\subsubsection{Feature calculation}

The most commonly used geometric parameters derived from LiDAR data for characterizing the structure of an object are height-related features (Koenig et al. 2015) (Table 2). In addition to these features, the local dimensionality features expressing how the neighborhood points are distributed have strong ability to discriminate different leaves and woods (Chi-Keung and Medioni 2002; Zheng et al. 2016b). In order to acquire the local dimensionality features, for a set of neighboring points within a radius of a given point, a local covariance matrix is calculated. The eigenvalues $(\lambda)$ are positive and ordered, so $\lambda_{1}>\lambda_{2}>\lambda_{3}$. The following features $\left(\alpha_{1 \mathrm{D}}, \alpha_{2 \mathrm{D}}, \alpha_{3 \mathrm{D}}\right)$ are selected to describe the likelihood that the shape of the local points of the given point is linear, planar, or random respectively (Demantke et al. 2011):

$$
\alpha_{1 D}=\left(\sqrt{\lambda_{1}}-\sqrt{\lambda_{2}}\right) / \sqrt{\lambda_{1}}, \alpha_{2 D}=\left(\sqrt{\lambda_{2}}-\sqrt{\lambda_{3}}\right) / \sqrt{\lambda_{1}}, \alpha_{3 D}=\sqrt{\lambda_{3}} / \sqrt{\lambda_{1}}
$$

Additionally, some radiometric features are proposed to including backscatter intensity (I), mean intensity of the local points $\left(\mathrm{I}_{\text {mean }}\right)$, intensity coefficient of variation of the local points $\left(\mathrm{I}_{\mathrm{cov}}\right)$, Riegl's pulse deviation $(\delta)$ and red, green and blue DNs from digital camera (RGB) (Table 5.2). Intensity has been proved to be useful in classification in many works (Lin and Herold 2016; Penasa et al. 2014; Zhang and Liu 2012). The distance effect on Riegl's intensity output has been eliminated by subtracting the range-dependent amplitude obtained when measuring to a reflectance standard. (Pfennigbauer and Ullrich 2010).

Table 5.2 List of the radiometric and geometric features used/ extracted in this study from TLS data

\begin{tabular}{|c|c|c|c|}
\hline Type & Feature & Definition & Reference \\
\hline \multirow{7}{*}{$\begin{array}{l}\text { Radiometric } \\
\text { features }\end{array}$} & I & Backscatter intensity & $\begin{array}{l}\text { Pfennigbauer and } \\
\text { Ullrich (2010) }\end{array}$ \\
\hline & $I_{\text {mean }}$ & Mean intensity of the local points & Koenig et al. (2015) \\
\hline & $\mathrm{I}_{\mathrm{cov}}$ & Intensity coefficient of variation of the local points & Koenig et al. (2015) \\
\hline & $\delta$ & Riegl's pulse deviation & $\begin{array}{l}\text { Pfennigbauer and } \\
\text { Ullrich }(2010)\end{array}$ \\
\hline & $\mathrm{R}$ & & \\
\hline & G & Red, green and blue DNs from digital camera & \\
\hline & B & & \\
\hline \multirow{6}{*}{$\begin{array}{l}\text { Geometric } \\
\text { features }\end{array}$} & $\alpha_{1 \mathrm{D}}$ & $\begin{array}{l}\text { The likelihood that the shape of the local points is } \\
\text { linear (1D) }\end{array}$ & Demantke et al. (2011) \\
\hline & $\alpha_{2 D}$ & $\begin{array}{l}\text { The likelihood that the shape of the local points is } \\
\text { planar (2D) }\end{array}$ & Demantke et al. (2011) \\
\hline & $\alpha_{3 \mathrm{D}}$ & $\begin{array}{l}\text { The likelihood that the shape of the local points is } \\
\text { random (3D) }\end{array}$ & Demantke et al. (2011) \\
\hline & StdZ & Standard deviation of the height values & Koenig et al. (2015) \\
\hline & Zdiff & Range of maximum and minimum height value & Koenig et al. (2015) \\
\hline & $\mathrm{Dz}$ & $\begin{array}{l}\text { Height difference between the single point and the } \\
\text { minimum elevation value }\end{array}$ & Koenig et al. (2015) \\
\hline
\end{tabular}




\subsubsection{Adaptive radius for near-neighbor search}

Many geometric and radiometric features relevant to LiDAR classification are based on the local neighborhood of each laser point (Penasa et al. 2014). For the fixed radius nearneighbor search, all points within this spherical neighborhood are included. For the classification of foliage and woody materials, the $\mathrm{DBH}$ of a tree is a good reference to choose a suitable searching radius (Ma et al. 2016a).

But the DBH varies across trees and plots. Thus, in this study, the dimensionality features were computed for increasing radius values $0.2 \mathrm{~m}, 0.3 \mathrm{~m}$ and $0.4 \mathrm{~m}$ based on the DBH variation in our plots from which we can use the adaptive radius search algorithm (Demantke et al. 2011) to choose the optimal one. The choice of the radius was a tradeoff between accuracy and computing time. A Shannon entropy of the discrete probability distribution proposed by Demantke et al. (2011) is calculated:

$$
E_{f}=-\alpha_{1 D} \ln \left(\alpha_{1 D}\right)-\alpha_{2 D} \ln \left(\alpha_{2 D}\right)-\alpha_{3 D} \ln \left(\alpha_{3 D}\right)
$$

The variable definition is detailed in Table 2. $E_{\mathrm{f}}$ is the entropy feature. The lower $\mathrm{E}_{\mathrm{f}}$ is, the more one dimensionality prevails over the two other ones. The optimal radius is obtained when:

$$
r_{\text {optimal }}=\operatorname{argmin}\left(E_{f}\right)
$$

The criterion allows defining an optimal radius that minimizes the entropy feature. So we calculate the entropy feature for the three radiuses and choose the radius with the lowest entropy feature. We are able to derive the optimal radius for each point.

\subsubsection{Classification}

In this study, we employed a nonparametric machine learning algorithm, i.e. Random Forests, since parametric classifiers such as Maximum Likelihood Classification (MLC) have a limitation when dealing with multi-modal input datasets (Liu et al. 2011). Random Forests is an ensemble classifier that has become popular within the remote sensing community. It can handle high data dimensionality with highly correlated features and is both fast and insensitive to overfitting (Belgiu and Drăguţ 2016). Random Forests classifiers have been widely applied in forest applications using hyperspectral and LiDAR data (Belgiu and Drăguţ 2016; Bigdeli et al. 2015; Koenig et al. 2015). Features importance from random forests was assessed using the learning classifier itself which was used to select relevant features. The implementation of classification was based on Scikit-learn python package (Pedregosa et al. 2011).

To train the Random Forests model, training samples were manually selected from the point cloud and were identified as foliage, woody and ground points (about 1000 per 
class). All the geometric and radiometric features listed in Table 2 were calculated with the various (near-neighbor) radii used for classification. These features were used as inputs in the Random Forests classifier. The class weights were 'balanced' for all instances, since the sample size of these classes was not equal. The number of trees was set to 100 (Guan et al. 2013; Pal 2005), and the number of features to consider when looking for the best split was set at default (the squared root of the total number of predictors). Consequently, the model was applied on the whole validation dataset (Fig. 5.1).

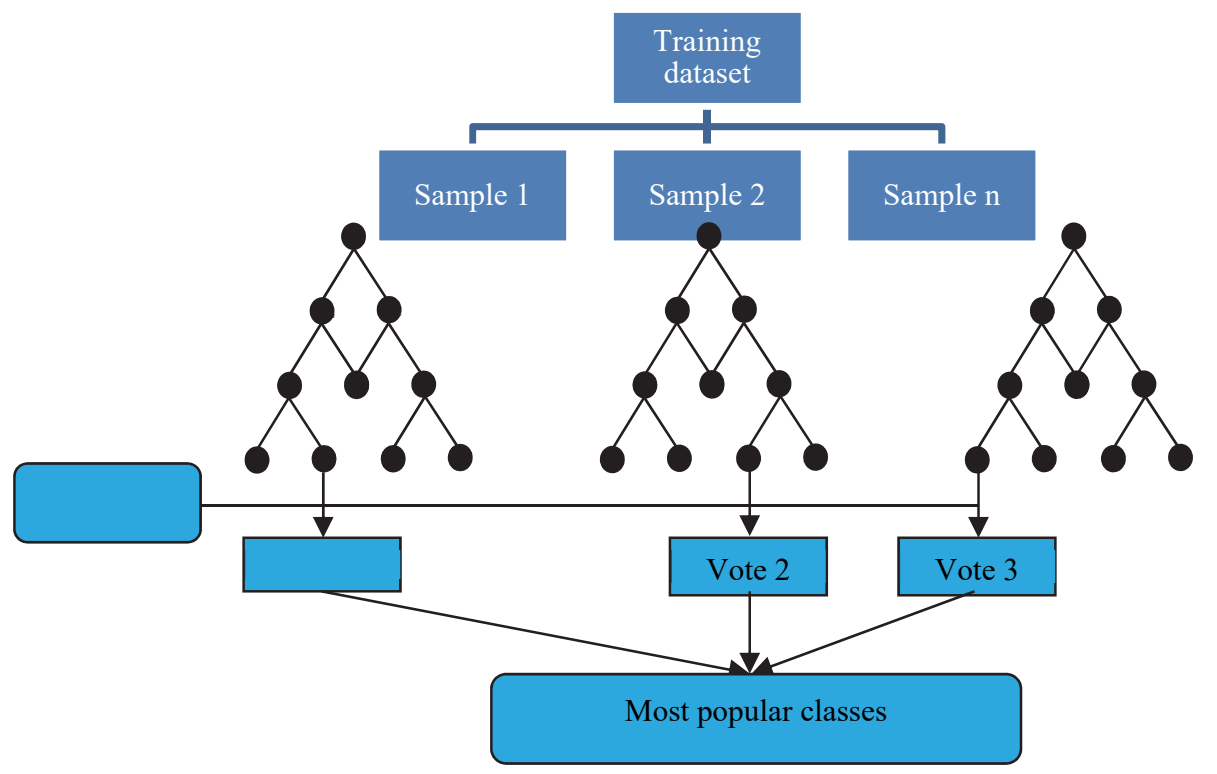

Figure 5.1 The flow chart of Random forests

\subsubsection{Accuracy assessment}

The validation was performed at two levels. First, we randomly selected 50 points per plot which were visually identified into the three different classes (viz. wood, foliage, ground) in LiDAR point cloud visualization. The point color was taken from the true color channel of the camera images for better visualization. Different classes could be easily identified in the point cloud visualization (Fig. 5.2). Second, we classified the images from the hemispherical camera for comparison to the classification results from TLS data. 

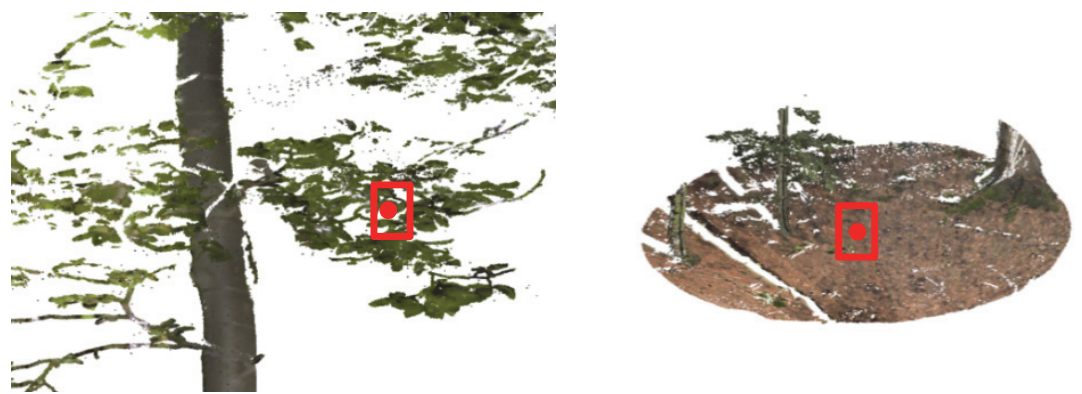

Figure 5.2 Visual interpretation of leaf and ground points

The accuracy assessment included overall accuracy, Cohen's kappa. A McNemar's test (de Leeuw et al. 2006) was conducted to compare the difference between classification results. The comparison of the classification results between TLS data and hemispheric photos was evaluated by a linear correlation test (Pearson 1896).

In order to test the classification accuracy of this method and analyze the factors that contribute to the variation of classification accuracy, we applied the method on ten plots individually and calculated the slope and understory coverage of each plot. We derived the slope by fitting a plane to the ground to calculate the angle between the plane and the horizontal plane (Gross and Thoennessen 2006). As for the understory coverage, we filtered all the points above $2 \mathrm{~m}$ (Martinuzzi et al. 2009), and estimated the coverage by dividing nonground points by all points. A Spearman's partial correlation (Kendall 1946) was used to test the influence of these two factors.

\section{$5.4 \quad$ Results}

\subsubsection{Geometric and radiometric features analysis}

Fig. 5.3 demonstrates the LiDAR intensity image of a subplot, showing the differences in intensity between the foliage, wood, and ground. The separability of intensity between foliage and other classes is illustrated in Fig. 5.4a. The foliage had a lower intensity than the other two classes. In Fig. 5.4b, the surface feature $\left(\alpha_{2 \mathrm{D}}\right)$ shows that the wood had the lowest likelihood of having a planar shape. A clear division can be seen between wood and the other two classes. However, although the ground had the highest values of $\alpha_{2 \mathrm{D}}$, the separability of the ground from foliage was not clear. Combining the mean intensity feature, the three classes could be better identified. 


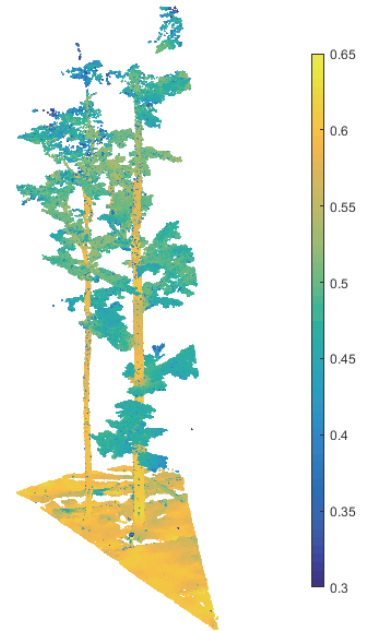

Figure 5.3 LiDAR Intensity image of a sample subplot

(a)

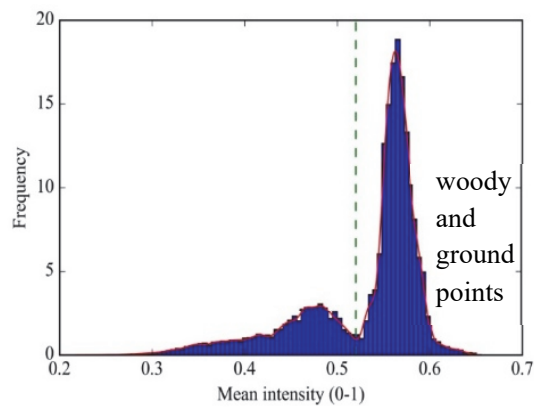

(b)

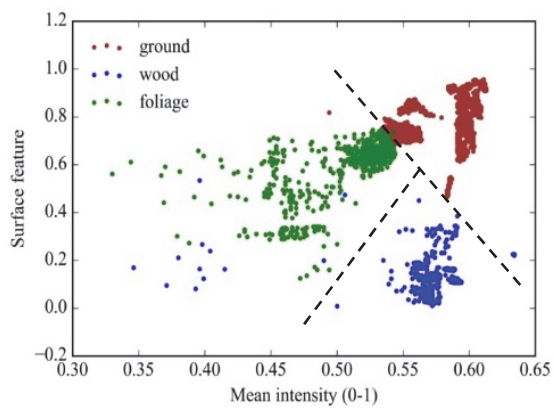

Figure 5.4 Distribution of LiDAR point clouds for different features; (a) frequency distribution of mean intensity data, (b) distribution of mean intensity and surface feature of point clouds

The importance of the features calculated using the Random Forests function is detailed in Table 5.3. 'Importance' can be used to predict the explanatory power of the features. The most relevant features were three geometric $\alpha_{2 \mathrm{D}}, \alpha_{3 \mathrm{D}}, \mathrm{Zdiff}, \mathrm{I}_{\text {mean }}$ and $\mathrm{I}_{\text {cov }}$ with values above 0.1 indicating geometric features were important than radiometric features.

Table 5.3 Feature importance values derived from Random Forests classifier ( : smaller than 0.02)

\begin{tabular}{cccccccccccccc}
\hline Features & $\alpha_{2 \mathrm{D}}$ & $\alpha_{3 \mathrm{D}}$ & Zdiff & $\mathrm{I}_{\text {mean }}$ & $\mathrm{I}_{\text {cov }}$ & $\mathrm{DZ}$ & $\mathrm{StdZ}$ & $\alpha_{1 \mathrm{D}}$ & $\mathrm{I}$ & $\mathrm{B}$ & $\mathrm{R}$ & $\mathrm{G}$ & $\delta$ \\
\hline Importance & 0.187 & 0.165 & 0.150 & 0.116 & 0.111 & 0.077 & 0.071 & 0.063 & 0.029 & $\sim$ & $\sim$ & $\sim$ & $\sim$ \\
\hline
\end{tabular}

The overall accuracy and kappa values for the classification results using different features for the ten plots are listed in Table 5.4. The performance of classification varied across different plots, but the classification using the combination of geometric and 
radiometric features outperformed that of using either of them alone which also is visually apparent in Fig. 5.5.

Table 5.4 Classification performance of using different features

\begin{tabular}{lllllllll}
\hline Plot & $\begin{array}{l}\text { Combination } \\
\text { features }\end{array}$ & of both & Geometric features & \multicolumn{3}{c}{ Radiometric features } \\
\hline & Overall & Kapp & Overall accuracy & Kapp & Overall accuracy & Kapp \\
& accuracy (\%) & a & $(\%)$ & a & $(\%)$ & a \\
1 & 90 & 0.85 & 78 & 0.67 & 56 & 0.34 \\
2 & 86 & 0.77 & 64 & 0.48 & 78 & 0.66 \\
3 & 86 & 0.77 & 60 & 0.40 & 54 & 0.30 \\
4 & 80 & 0.68 & 80 & 0.68 & 18 & 0.06 \\
5 & 82 & 0.72 & 48 & 0.22 & 48 & 0.24 \\
6 & 82 & 0.73 & 74 & 0.61 & 50 & 0.25 \\
7 & 90 & 0.83 & 72 & 0.56 & 26 & 0.01 \\
8 & 84 & 0.73 & 74 & 0.55 & 34 & 0.15 \\
9 & 80 & 0.7 & 78 & 0.66 & 50 & 0.21 \\
10 & 84 & 0.75 & 76 & 0.61 & 44 & 0.22 \\
\hline
\end{tabular}

(a)

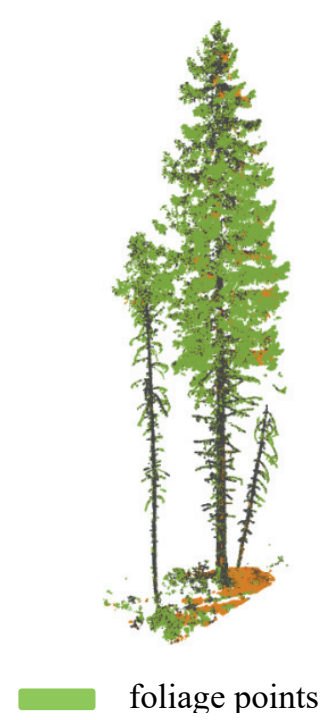

(b)

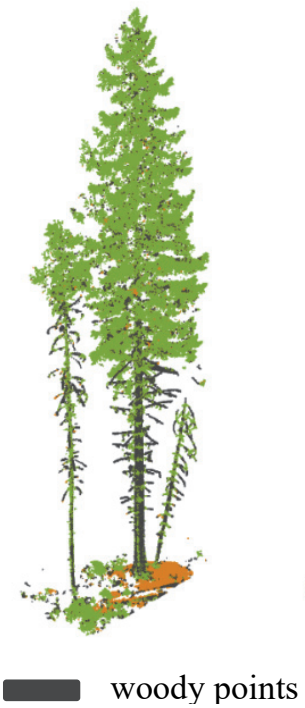

(c)

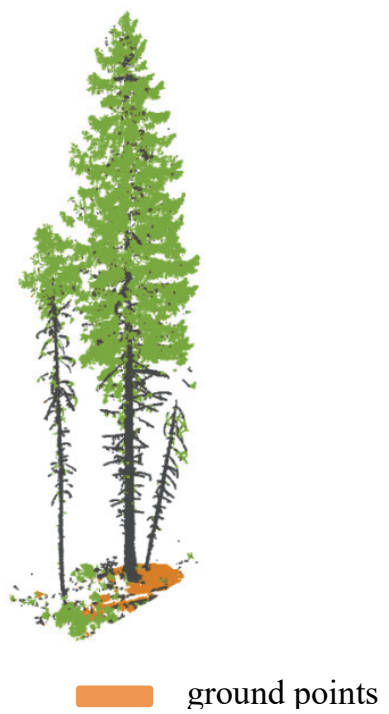

Figure 5.5 Visual comparison of classification results based on (a) radiometric features, (b) geometric features, and (c) the combination of both radiometric and geometric features 


\subsubsection{Effects of searching radius on classification performance}

In order to evaluate the effects of using different searching radiuses, we calculated the overall accuracy and Cohen's kappa of the classification results for the ten plots with the combination of both geometric and radiometric features (Table 5.5). The results obtained with the fixed-radius plots were generally high, the plot-to-plot results varied, while the adaptive radius results were consistently high for all the plots.

Table 5.5 Classification performance of using different searching radiuses

\begin{tabular}{|c|c|c|c|c|c|c|c|c|}
\hline \multirow[b]{2}{*}{$\begin{array}{l}\text { Pl } \\
\text { ot }\end{array}$} & \multicolumn{2}{|c|}{ Adaptive radius } & \multicolumn{2}{|c|}{ Radius: $0.2 \mathrm{~m}$} & \multicolumn{2}{|c|}{ Radius: $0.3 \mathrm{~m}$} & \multicolumn{2}{|c|}{ Radius: $0.4 \mathrm{~m}$} \\
\hline & $\begin{array}{l}\text { Overall } \\
\text { accurac } \\
\text { y }(\%)\end{array}$ & $\begin{array}{l}\text { Kap } \\
\text { pa }\end{array}$ & $\begin{array}{l}\text { Overall } \\
\text { accurac } \\
\text { y (\%) }\end{array}$ & Kappa & $\begin{array}{l}\text { Overall } \\
\text { accuracy } \\
(\%)\end{array}$ & Kappa & $\begin{array}{l}\text { Overall } \\
\text { accuracy } \\
(\%)\end{array}$ & Kappa \\
\hline 1 & 90 & 0.85 & 86 & 0.79 & 84 & 0.76 & 84 & 0.76 \\
\hline 2 & 86 & 0.77 & 72 & 0.57 & 80 & 0.66 & 80 & 0.66 \\
\hline 3 & 86 & 0.77 & 84 & 0.74 & 82 & 0.71 & 84 & 0.74 \\
\hline 4 & 80 & 0.68 & 68 & 0.52 & 60 & 0.39 & 64 & 0.49 \\
\hline 5 & 82 & 0.72 & 64 & 0.39 & 64 & 0.40 & 64 & 0.40 \\
\hline 6 & 82 & 0.73 & 68 & 0.52 & 78 & 0.67 & 72 & 0.58 \\
\hline 7 & 90 & 0.83 & 84 & 0.75 & 68 & 0.49 & 80 & 0.67 \\
\hline 8 & 84 & 0.73 & 74 & 0.61 & 68 & 0.49 & 64 & 0.43 \\
\hline 9 & 80 & 0.70 & 62 & 0.42 & 58 & 0.35 & 56 & 0.33 \\
\hline 10 & 84 & 0.75 & 62 & 0.45 & 48 & 0.22 & 52 & 0.30 \\
\hline
\end{tabular}

Fig. 5.6 shows the classification results using different searching radius in two sample plots. In plot 1 , the misclassification appeared for the results of using the radiuses of 0.2 $\mathrm{m}$ and $0.4 \mathrm{~m}$, whereas the misclassification in plot 2 emerged for the results of using the radiuses of $0.2 \mathrm{~m}$ and $0.3 \mathrm{~m}$. As can be observed from Fig. 5.6 the best fixed radius differed for various plots and trees, while the adaptive radius showed its robustness across different plots. 
(a)

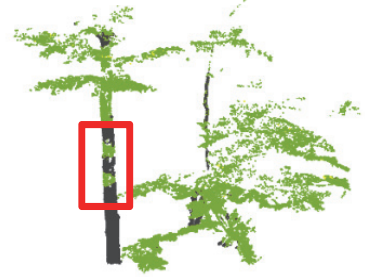

(c)

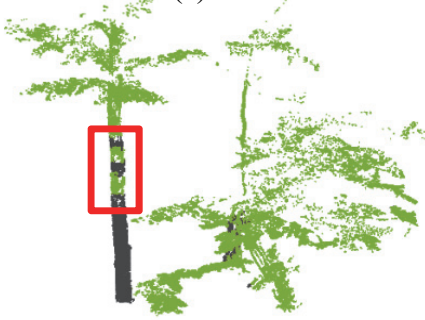

(b)

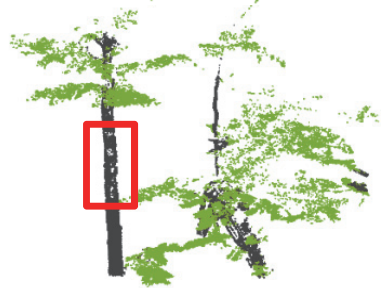

(d)

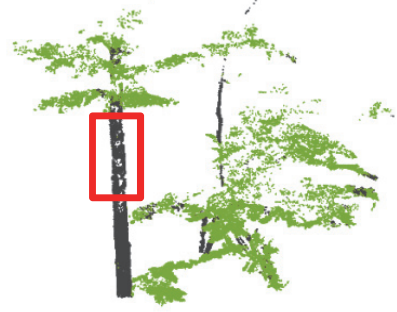

(1) Plot 1

(b)

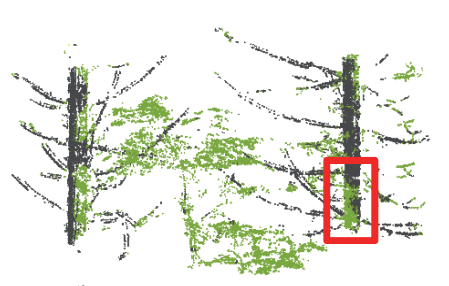

(c)

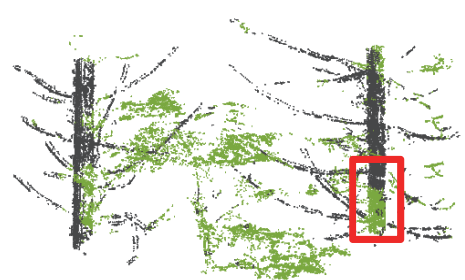

(d)

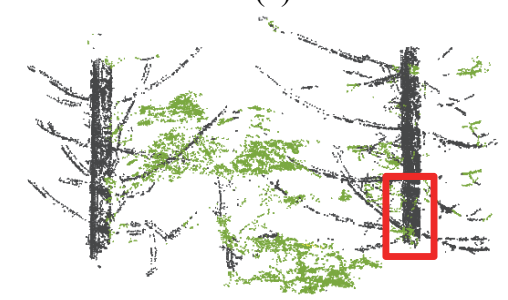

(2) Plot 2

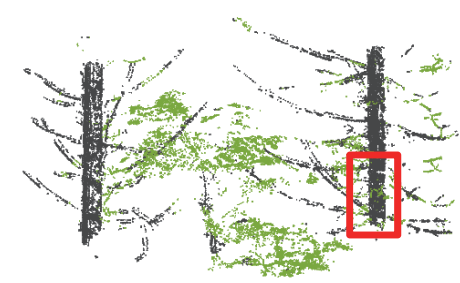

foliar points

woody points

Figure 5.6 Visual comparison of classification results using based on different searching radius for two sample plots; (a) $r=0.2 \mathrm{~m}$, (b) $\mathrm{r}=0.3 \mathrm{~m}$, (c) $\mathrm{r}=0.4 \mathrm{~m}$ and (d) adaptive radius 


\subsubsection{Impact of understory and slope on classification performance}

Table 5.6 shows that the overall accuracy of all the ten plots was above $80 \%$. The Spearman's partial correlation test between the slope, understory coverage, and the accuracy demonstrated (Table 5.7) that both, the slope and understory coverage had significant negative effect on the overall classification accuracy with an R value of -0.79 and -0.76 . The overall accuracy decreased with increasing understory coverage and slope.

Table 5.6 Classification results and statistics for the sample plots

\begin{tabular}{|c|c|c|c|c|c|c|c|c|}
\hline $\begin{array}{c}\text { Plo } \\
\mathrm{t}\end{array}$ & $\begin{array}{l}\text { Dominan } \\
\text { t species }\end{array}$ & $\begin{array}{l}\text { Slop } \\
\text { e }\left(^{\circ}\right)\end{array}$ & $\begin{array}{c}\text { Understor } \\
\text { y coverage } \\
(\%)\end{array}$ & $\begin{array}{c}\text { Overall } \\
\text { accurac } \\
\text { y }(\%)\end{array}$ & $\begin{array}{c}\text { Kapp } \\
\text { a }\end{array}$ & $\begin{array}{l}\text { Ground } \\
\text { accurac } \\
\text { y }(\%)\end{array}$ & $\begin{array}{l}\text { Wood } \\
\text { accurac } \\
\text { y }(\%)\end{array}$ & $\begin{array}{c}\text { Leaf } \\
\text { accurac } \\
\text { y }(\%)\end{array}$ \\
\hline 1 & Beech & 3.6 & 32 & 90 & 0.85 & 100 & 82 & 93 \\
\hline 2 & Spruce & 3.1 & 66 & 86 & 0.77 & 71 & 92 & 90 \\
\hline 3 & $\begin{array}{l}\text { Beech, } \\
\text { Spruce }\end{array}$ & 4.6 & 39 & 86 & 0.77 & 83 & 87 & 100 \\
\hline 4 & Spruce & 4.9 & 53 & 80 & 0.68 & 91 & 71 & 75 \\
\hline 5 & Beech & 11.3 & 50 & 82 & 0.72 & 93 & 71 & 82 \\
\hline 6 & $\begin{array}{l}\text { Beech, } \\
\text { Spruce, } \\
\text { Fir }\end{array}$ & 12.0 & 49 & 82 & 0.73 & 82 & 71 & 94 \\
\hline 7 & $\begin{array}{l}\text { Beech, } \\
\text { Spruce }\end{array}$ & 6.0 & 39 & 90 & 0.83 & 96 & 93 & 67 \\
\hline 8 & $\begin{array}{l}\text { Beech, } \\
\text { Spruce }\end{array}$ & 16.6 & 29 & 84 & 0.73 & 96 & 87 & 44 \\
\hline 9 & Beech & 18.8 & 41 & 80 & 0.70 & 87 & 75 & 82 \\
\hline 10 & Beech & 3.4 & 42 & 84 & 0.75 & 91 & 73 & 83 \\
\hline
\end{tabular}

Table 5.7 Spearman's partial correlation test between the slope, understory coverage and the accuracy (bold: $\mathrm{P}$ value $<0.05$ )

\begin{tabular}{ccc}
\hline & Understory coverage & Slope \\
\hline Overall accuracy & $\mathbf{R}=\mathbf{- 0 . 7 6 ,}, \mathbf{P}=\mathbf{0 . 0 2}$ & $\mathbf{R}=\mathbf{- 0 . 7 9 ,}, \mathbf{P}=\mathbf{0 . 0 1}$ \\
Ground accuracy & $\mathrm{R}=-0.62, \mathrm{P}=0.08$ & $\mathrm{R}=-0.05, \mathrm{P}=0.89$ \\
Wood accuracy & $\mathrm{R}=-0.54, \mathrm{P}=0.14$ & $\mathrm{R}=-0.43, \mathrm{P}=0.25$ \\
Leaf accuracy & $\mathrm{R}=-0.01, \mathrm{P}=0.97$ & $\mathrm{R}=-0.41, \mathrm{P}=0.28$ \\
\hline
\end{tabular}

\subsubsection{Comparison with hemispherical image classification}

We classified images from the hemispherical camera into foliage, woody and sky pixels and estimated a ratio between foliage and woody pixels. This ratio was plotted against that derived from TLS classification. Fig. 5.7 shows that there was a good correlation between the results. TLS underestimated the ratio of foliage and woody materials compared to hemispherical photos. In Fig. 5.8 the final classification result for a mixed plot compared to LiDAR point cloud colorized by RGB photos is presented. 


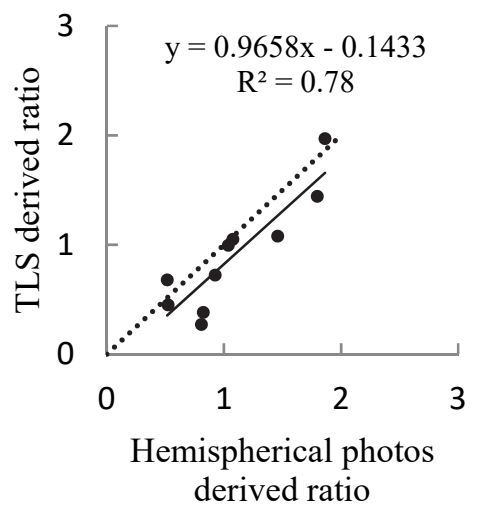

Figure 5.7 Comparison of the ratio between foliage and woody materials derived from TLS and hemispherical photo for the 10 sample plots

(a)

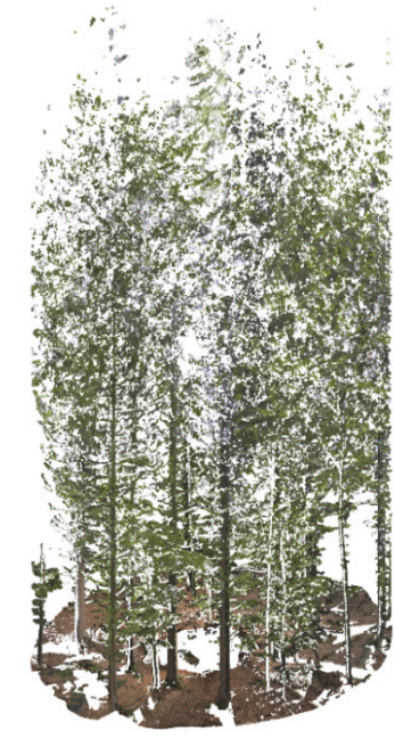

foliage points (b)

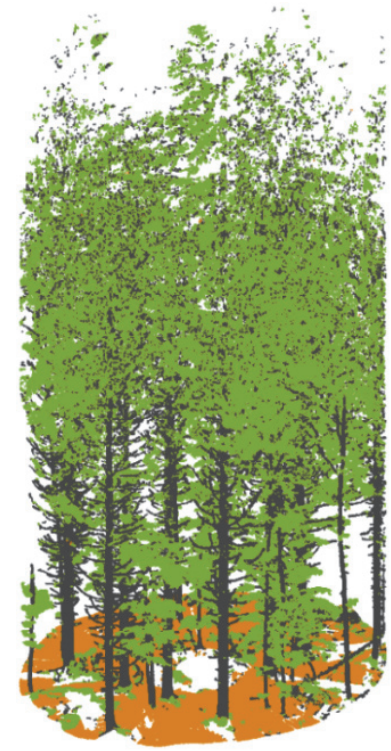

woody points

Figure 5.8 TLS point cloud colorized by RGB photos and its final classification result for a mixed plot; (a) TLS point cloud and (b) classification result

\subsection{Discussion and conclusion}

In this paper, we used an adaptive radius near-neighbor search algorithm to obtain both geometric and radiometric features for discriminating between foliage, woody and ground 
materials from TLS data. We achieved an average overall accuracy of $84.4 \%$ and an average kappa value of 0.75 .

The average overall accuracy of using either geometric features or radiometric features alone was $70.4 \%$ and $45.8 \%$ respectively. Coupling radiometric and geometric information provided a better separability between foliage, woody and ground points than using either of them alone (Table 4). For instance, in the geometric domain, the wood could be easily separated from the ground and foliage in a broadleaf plot due to its nonsurface feature (Fig. 5.4b). However, broadleaves could have the similar geometric feature (surface feature) to the ground (Fig. 5.4b). Intensity provides the possibility to differentiate leaves, thanks to their different spectral characteristics at the water absorption wavelength $(1550 \mathrm{~nm})$. Leaf tissues usually have much lower intensity than woody tissues and ground at this wavelength, as a considerable amount of the energy emitted from TLS is absorbed by the leaf water (Zhu et al. 2015). But the use of intensity data is usually limited by the influence of the distance, partial hit and laser incidence angle (Kaasalainen et al. 2011). The distance effect was eliminated for this instrument, while the partial hit and incidence angle effects are very complicated in this situation. Often in a forest plot, some targets like small branches and needles are irregular and also smaller than the footprint of the scanner, which makes it difficult to estimate the incidence angle and the collision area between the footprint and target. A possible solution to this issue is to use a multi spectral TLS. If the influence of the incidence angle and the collision area of the target are similar at two wavelengths, the ratio would be insensitive to these factors (Gaulton et al. 2013). Combining multi spectral information with geometric information may largely improve the discrimination of different materials in the forest.

The results demonstrated that the adaptive radius near-neighbor search performs robustly comparing to the fixed radius near-neighbor search in the classification between foliage, wood, and ground across different plots (Table 5.5). The average overall accuracy of using the fixed radius was $70.5 \%$ which much lower than using the adaptive radius. One important factor could be that the dimensionality of a local cluster of points varies as the searching radius changes (Brodu and Lague 2012). For instance, the point cloud of a stem at a few centimeter scale could have a surface feature (2D), while at a larger scale, the point cloud of the stem is more likely to have a linear feature (1D). Fig. 5.6 confirms that using fixed radiuses was not the most reliable choice, since the best radius varied in different situations. Combining features from all scales leads to high dimensional data and low computational efficiency, since it produces much more features than using one scale. The adaptive radius search was originally proposed to classify between buildings and vegetation in an urban area at a larger scale (Demantke et al. 2011). It chooses the optimal scale at different situations while avoiding having high dimensional data. The application of this method on the classification of foliage, woody and ground points at a fine scale shows its superiority over the fixed radius search across species and structure. The choice of using the radiuses of $0.2 \mathrm{~m}, 0.3 \mathrm{~m}$, and $0.4 \mathrm{~m}$ was a tradeoff between 
accuracy and computational efficiency. Choosing the optimal radius from more scales of radius might improve the result and can be more robust in various forest situations at a larger scale.

The classification results of the sample plots showed that both slope and understory coverage were significantly correlated to classification accuracy. The accuracy decreased with increasing understory coverage (Table 5.6). In areas with high understory coverage percent, classification could not perform well to separate ground and vegetation points (Huang et al. 2011). Thick vegetation cover makes LiDAR more difficult to penetrate through it to the ground (Hodgson and Bresnahan 2004). The slope also had a negative impact on the classification results. In this study, we have used some features related to height (i.e. StdZ, Zdiff and Dz). These parameters have the ability to discriminate the ground from foliage and wood in flat areas (Koenig et al. 2015), since the height difference of the ground points is much smaller than the other two classes. However, in the plots with complex topography (e.g. plot 9), they become less reliable. In addition, due to the variation of the incidence angle between the ground and laser beam, the intensity of ground points could be lower, further affecting the separation between the foliage, wood, and ground (Tang et al. 2014b).

It is useful to compare our results with those from previous studies, although the forest structure and forest condition vary a lot and the classification methods used earlier are different. Zheng et al. (2016a) used a point classification method to classify foliar, woody and ground points, resulting in an overall accuracy of $85.5 \%$ for one plot. In another study, Ma et al. (2016b) applied a geometric-based automatic forest point classification algorithm to three plots in Washington Park Arboretum and some individual trees in a forest. The overall accuracy of the three plots were $84.3 \%, 94.4 \%$, and $97.8 \%$, respectively. The difference in accuracy can be attributed to several factors. Our study area is located in a mixed natural forest which is more complicated in forest structure, condition and topography. The variation of slope in their study area ranged from $0^{\circ}$ to $10^{\circ}$, much less than our study area (i.e., $3.4^{\circ}$ to $18.8^{\circ}$ ). There was also little understory which showed a negative relationship with the classification accuracy (Table 5.7). In addition, Ma et al. (2016b) showed that the spatial distribution patterns of foliage elements violated the feature definition of leaves in a plot dominated by Douglas fir, thus the method provided poor classification results. It was suggested by the authors to incorporate additional information such as calibrated intensity information (Ma et al. 2016b). In plot 6 in our study, Douglas fir was one of the three main species. An overall accuracy of $82 \%$ and leaf accuracy of $94 \%$ were achieved. However, the percentage of Douglas fir was much lower than that in their study area. Further research is needed to assess the species influence at a larger scale.

The correlation between classifications results obtained using TLS data and hemispherical images was strong, although the one from TLS underestimated the foliage 
material by $17.2 \%$. The images from the hemispherical camera are more subject to the occlusion effect by leaves which were more scattered because of its 2D nature. Therefore, leaf pixels in these images might be overestimated. TLS images from four scan positions, in this case, is less affected by occlusion. Additionally, the images from the hemispherical camera (with a hemispherical view) cover all the objects within the field of view, while TLS only records objects around the scanner within a certain distance (cylinder field of view). This could also cause the discrepancy of the results.

In the Bavarian Forest National Park, there is little human intervention. "Let nature be nature!" is the philosophy of the Bavarian Forest National Park. As a result, some plots were found not only covered by low understory vegetation, but also lying dead wood and dead leaves. This could significantly change the geometric dimensionality and lower the intensity value of the ground. Flat soil ground usually has a surface feature (Demantke et al. 2011), while ground cover with dead leaves can has a random feature, depending on the thickness of the dead leaves on the ground. Therefore, the true ground becomes much more indistinguishable from vegetation components. In addition, the slope of some plots (e.g. plot 9) is steep, which makes it more difficult to differentiate the ground from the other classes. A quantitative assessment of these interactions needs to be further studied, but the relative impact of these interaction effects on the overall accuracy when distinguishing woody from photosynthetic foliar material is most probably marginal. 
Chapter 6

Synthesis: LiDAR and hyperspectral remote sensing of leaf water content 


\subsection{Summary}

Forests cover a third of all land on earth which provide various ecosystem services. The assessment and prediction of forest change and its influence on forest ecosystems require the monitoring of a wide range of essential biodiversity variables. Leaf water content (LWC) as one of the variables plays an important role in mapping and monitoring the condition of forest ecosystems, including environmental stresses, fire susceptibility and species distribution (Asner et al. 2016; Ustin et al. 1998; Yi et al. 2014). As a consequence, water content was proposed as one of the essential biodiversity variables (EBVs) to assess progress towards the Aichi Biodiversity Targets (Skidmore et al. 2015). It is also considered as a very important variable for developing fire susceptibility models by the Global Observation of Forest and Land Cover Dynamics fire implementation team (GOFC/GOLD-Fire) (Chuvieco and Kasischke 2007).

This thesis evaluated the usage of remote sensing data for the estimation of LWC at different scales and examined the impact of the incidence angle and partial hits on LiDAR intensity data, as well as eliminated the influence of canopy structural variables and background reflectance on canopy spectral reflectance. Chapter 2 investigated the influence of the incidence angle and leaf surface feature on the relationship between LWC and terrestrial laser scanner (TLS) backscatter intensity. The accuracy of LWC estimation was significantly improved by applying a reference target-based model using the LiDAR intensity. Chapter 3 examined the partial hits and incidence angle effects on LWC estimation at the individual canopy scale. LWC vertical distribution within plant canopy can be retrieved from TLS. Chapter 4 evaluated the integration of LiDAR and hyperspectral data for the estimation of LWC through radiative transfer models (RTM) at the regional scale. The results showed that the introduction of canopy cover and background reflectance using the integration of LiDAR and hyperspectral data significantly improved the accuracy of LWC retrieval. Chapter 5 assessed the performance of the combination of geometric and radiometric features and an adaptive radius near neighbor search method on the classification of foliar and woody materials using TLS. The findings suggested that an accurate classification between foliar and woody materials can be achieved in a mixed natural forest by using TLS.

\subsection{Leaf water content estimated at the leaf scale}

Most water content studies at the leaf scale have focused on the use of hyperspectral data. Estimation using LiDAR has rarely been explored since LiDAR intensity is significantly affected by the distance and incidence angle effects. Unlike ALS, the distance effect on TLS intensity is mostly dependent on instrumental factors, which is constant for a specific instrument, so the calibration is usually direct (Kaasalainen et al. 2011). However, the incidence angle effect on TLS intensity is strongly associated with surface properties (Krooks et al. 2013). The experiment showed that even when two species had the same LWC, their intensity could be different at the perpendicular angle (Fig. 6.1). For example, 
Photinia fraseri and Ficus benjamina both had around an average LWC of $0.01 \mathrm{~g} / \mathrm{cm}^{2}$, but the intensity was significantly different (Fig. 6.1a). The main reason behind this is that the surface properties (Eitel et al. 2010a) were different. The backscatter intensity reflected from the leaves consists of two parts: the specular intensity and the diffuse intensity. The diffuse intensity is mainly dependent on LWC, whereas the specular intensity is determined by the surface properties. The linear combination of the Lambertian model and Beckmann law successfully simulated the fraction of the specular intensity and the diffuse intensity (Table 6.1). Ficus benjamina had a higher specular fraction (0.56) than Photinia (0.36), so the specular intensity caused by the surface was also higher. The removal of the specular intensity was achieved so that LWC became the main factor that contributed to the recorded intensity (Fig. 6.1b). The reflectance model for intensity correction is desirable, especially for data located in the nadir regions of targets with highly reflective surfaces (Ding et al. 2013).

a

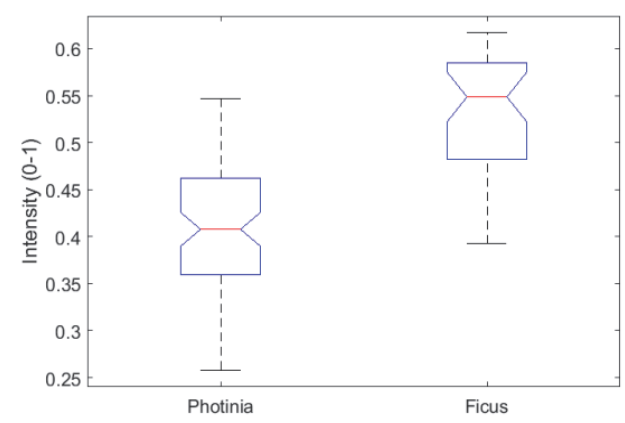

b

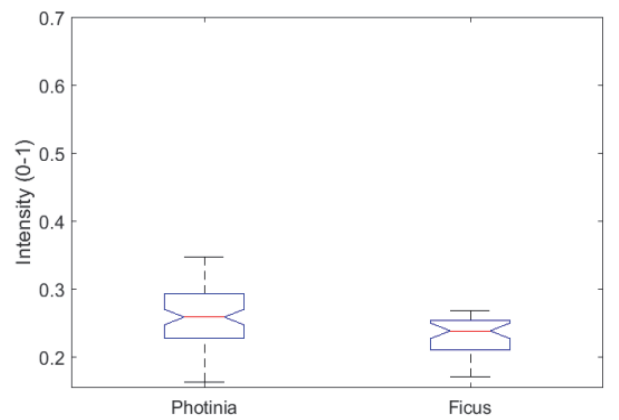

Figure 6.1 The backscatter intensity of two species with same leaf water content. (a) Before removal of the specular intensity and (b) after removal of the specular intensity.

Table 6.1 Terrestrial laser scanner backscatter intensity simulation for 2 plant species using a linear combination of the Lambertian model and Beckmann law. ks: specular fraction

\begin{tabular}{llll}
\hline Species name & $\mathrm{k}_{\mathrm{s}}$ & $R^{2}$ & RMSE \\
\hline Ficus benjamina & 0.56 & 0.98 & 0.01 \\
Photinia fraseri & 0.36 & 0.94 & 0.02 \\
\hline
\end{tabular}

TLS has been demonstrated in numerous studies for its ability to capture vegetation structure, while its potential for LWC estimation has rarely been explored. It offers the advantage to avoid many intrinsic drawbacks of passive remote sensing, by explicitly being able to model and account for the canopy structure influence, viewing and illumination geometry and the noise from background and woody materials (Magney et al. 2014). Additionally, it can capture the detailed LWC distribution within leaves and canopy (Fig. 6.2). Fig. 6.2a shows that the water content of the venation is higher than the other parts since the principal function of the venation is to deliver water (Roth- 
Nebelsick et al. 2001). In Fig. 6.2b, the older leaves had lower LWC than emerging leaves. Mooney et al. (1977) demonstrated that when leaves are fully hydrated, new leaves have a higher water content than older leaves. During drought-stress, plants water is redistributed from old to young leaves to maintain the water content of young leaves, and thereby leaf function (EVANS 1932; Rabas and Martin 2003). Kramer (1959) stated that young leaves usually can obtain water at the expense of older leaves and the latter usually die first during drought. These two maps indicate that TLS has a great potential to assess plant physiological status. Due to the powerful highly collimated laser light and the small footprint size, these measurements using TLS at the fine leaf scale do not require direct physical contact, which is the biggest advantage of TLS against passive remote sensing. With the advance of the technology, the beam divergence will be smaller so that even at a long distance (plot scale) TLS can capture the detailed information of a single leaf. It is more comprehensive, and cost and time efficient, holding promise to replace field measurements.

a

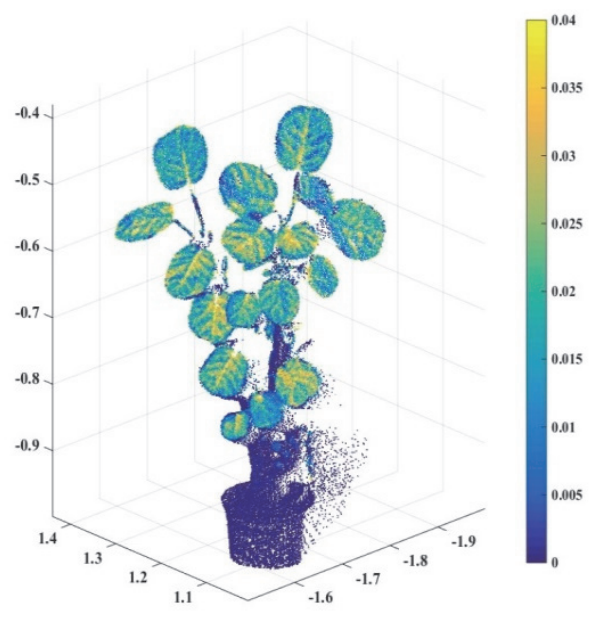

b

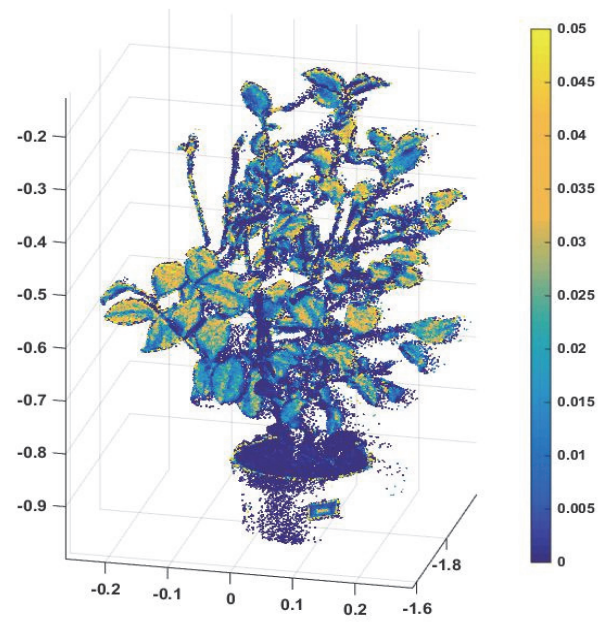

Figure 6.2 3D Leaf water content mapping $\left(\mathrm{g} / \mathrm{cm}^{2}\right)$. (a) Polyscias fabian and (b) Camellia japonica.

\subsection{Leaf water content distribution within the canopy of individual plants}

The distribution of LWC within the canopy affects the light penetration and scattering. It also plays an important role in the distribution of other plant biochemical parameters. But the spatial distribution within the canopy, especially the vertical distribution has never been studied using remote sensing. Chapter 3 investigated the feasibility of estimating LWC distribution within the canopy of individual plants using TLS by removing the effects of partial hits. It demonstrated for 
the first time that LWC distribution within the canopy of individual plants can be estimated with a good accuracy using TLS.

Chapter 2 showed that LWC can be modeled and estimated from the TLS backscatter intensity with a good accuracy at the leaf scale. Chapter 3 intended to upscale the use of TLS from the leaf level to the individual canopy level. In addition, instead of using discrete return data, a number of fullwaveform features were assessed for their ability to estimate LWC. The incidence angle effect remained an issue at the canopy scale since the zenith scan angle of the TLS was far from vertical. Fig. 6.3 shows the relationship between three full-waveform features (i.e., amplitude, backscatter cross-section, and backscatter coefficient) and measured LWC before correcting for the incidence angle effect. None of the three parameters showed a strong relationship with LWC, while the $R^{2}$ value between the backscatter coefficient and LWC was the highest. After the incidence angle correction, a strong and significant relationship $\left(R^{2}=0.63\right)$ emerged, indicating that the incidence angle correction was also effective for the canopy level correction. The logarithmic relationship showed that laser signal was insensitive to high LWC values. This problem also affected the LWC prediction. Fig. 6.5 shows the prediction of LWC for both excluding (a) and including the extreme case (b). Compared to the prediction of LWC $\left(R^{2}=0.72\right)$ excluding the extreme case with very high LWC, the $R^{2}$ value including the extreme case dropped to 0.66 (Fig. 6.5). This problem was also observed at the leaf level study. This requires further investigation.
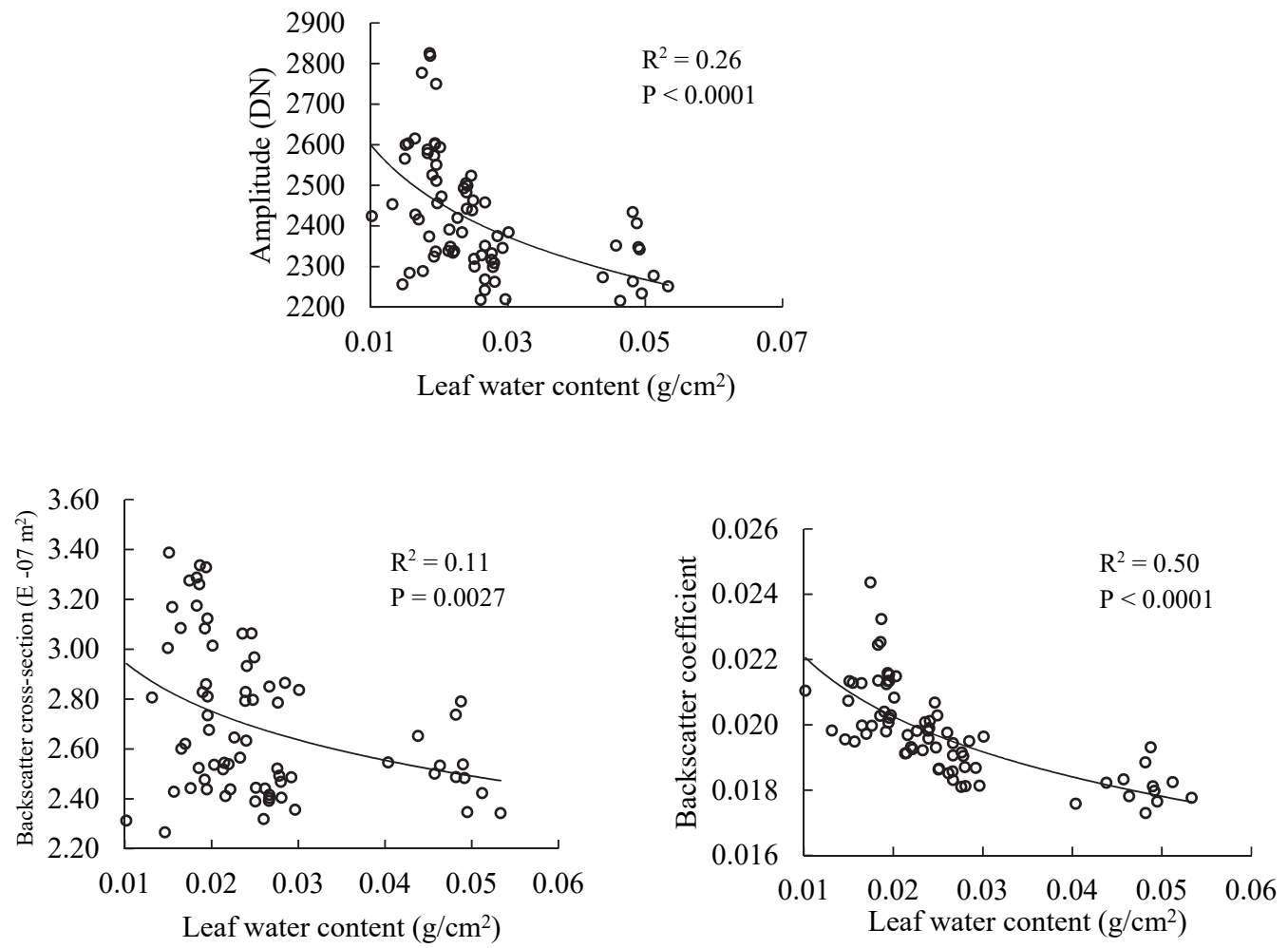

Figure 6.3 Relationships between three waveform parameters (i.e., amplitude, backscatter crosssection, and backscatter coefficient) and LWC before correcting for incidence angle effect. 


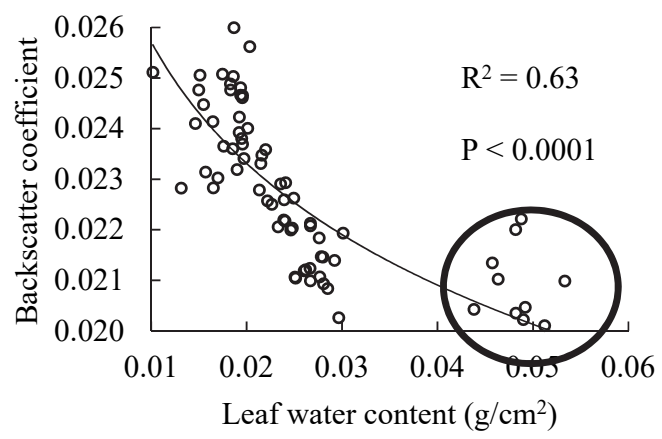

Figure 6.4 Relationships between the backscatter coefficient and LWC after correcting for incidence angle effect (The circled points indicate extremely high LWC).

a

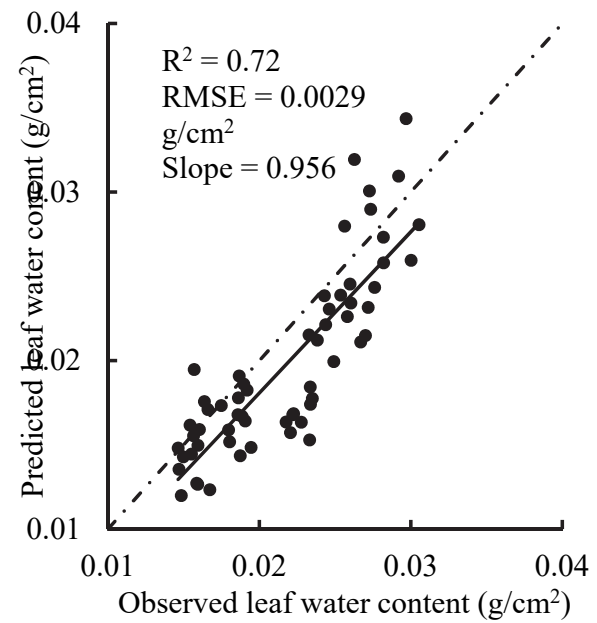

b

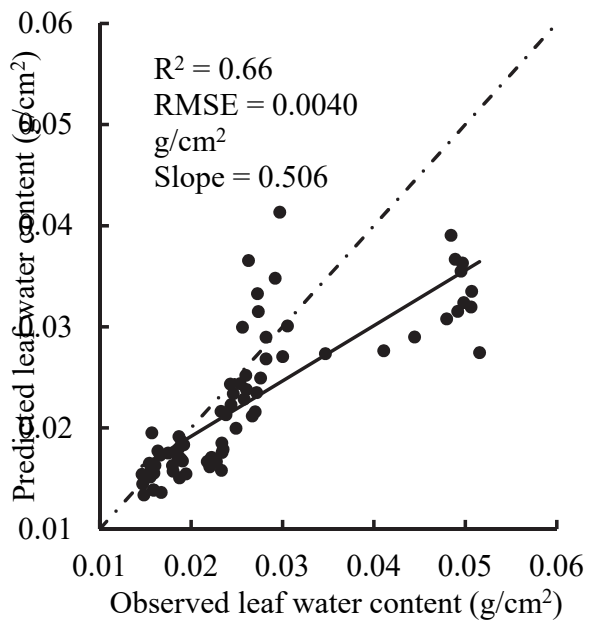

Figure 6.5 Scatterplot of the measured and estimated leaf water content by the backscatter coefficient. (a) excluding extreme case and (b) including extreme case

Fig. 6.6 showed that on some parts of the leaf edge, the LWC was slightly lower than that on the other parts of a leaf. We think this is due to the overcorrection of the incidence angle influence. On the edge of some leaves, the incidence angle was larger due to the irregular curling edge of the leaves. The overestimation of the incidence angle of the leaf edge could cause overcorrection, leading to higher backscatter coefficient values, thus lower LWC values. 

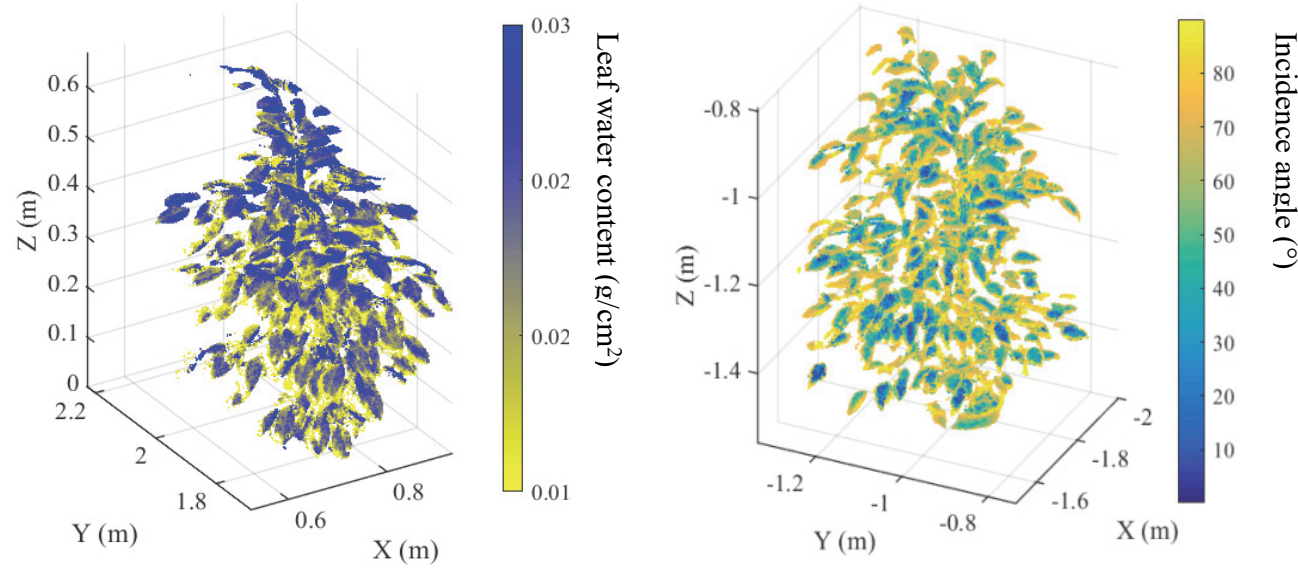

Fig. 6.6 Leaf water content and incidence angle mapping

An example of LWC mapping (Fig. 6.7) showed the LWC vertical distribution as well as the horizontal distribution within the canopy of a typical individual plant. Again, new leaves at the top of the plant showed a higher LWC than older leaves (Magney et al. 2014). This phenomenon might be helpful to determine whether a plant is under stress conditions such as drought. Moreover, it has been shown that in some radiative transfer models the vertical heterogeneity of biophysical and biochemical parameters within the canopy largely affects the canopy reflectance simulation and inversion (Wang and Li 2013). However, this is very difficult to measure with passive remote sensing data. Using LiDAR techniques, we are able to detect the LWC at different heights. They can provide very useful information for RTMs which incorporate the vertical LWC in the future.

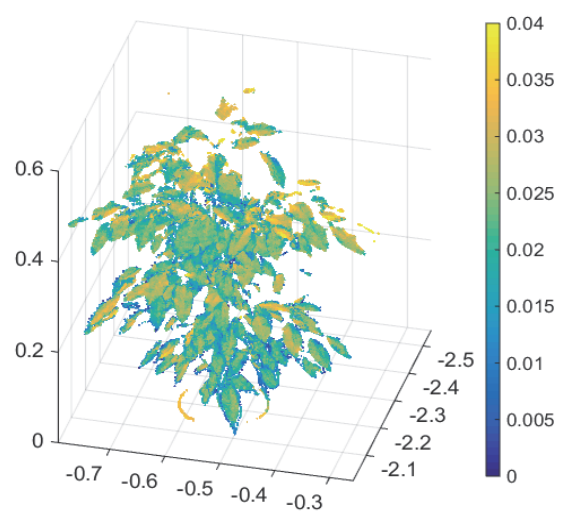

Figure 6.7 An example of leaf water content mapping 


\subsection{Leaf water content estimation at the regional scale}

The estimation of LWC at the regional scale using RTMs or statistical models may be confounded by canopy structure and forest background (Ali et al. 2016b; Darvishzadeh et al. 2008a). When using RTM, a common problem is the ill-posed problem when inverting a model, which is well documented in a number of studies (Combal et al. 2003; Koetz et al. 2005). It is mainly caused by the under-determined nature of the modeling schemes (Jacquemoud et al. 1995). The use of prior knowledge has been suggested by many studies to solve the ill-posed problem (Dasgupta et al. 2009; Xiao et al. 2014). However, at the regional scale, field measurements of such information are labor intensive and time-consuming, especially in remote areas, and there is a lot of temporal and spatial variation. LiDAR offers an alternative to obtaining information about vegetation structure and forest background. The combination of LiDAR and hyperspectral data may allow retrieval of background reflectance and canopy structure which can be used as prior information for LWC estimation - such an approach has not been earlier investigated.

Chapter 4 assessed the performance of the inversion of INFORM radiative transfer model using prior information obtained from airborne LiDAR and hyperspectral data for LWC estimation. The INFORM model was modified by replacing stem density and crown radius with the canopy cover since canopy cover could be effectively retrieved from airborne LiDAR data (Korhonen et al. 2011). The global sensitivity analysis shows that at the regional scale, the canopy cover (CC) (0.1-1) and background reflectance had a larger influence on canopy reflectance than LWC when there was no prior information (Fig. 6.8). The study demonstrated that, despite the large influence of the canopy cover and background reflectance, LWC can be estimated with a good accuracy through the inversion of INFORM model using prior information obtained from LiDAR and hyperspectral data.

In order to map the LWC of the Bavarian Forest National Park, the range of the canopy cover (Fig. 6.9) was set between 0.1-1 for lookup table (LUT) generation based on the FAO (Forest and Agriculture Organization of the United Nations) definition of forests (FAO 2017). This was the main reason why canopy cover had such a large influence on the variation of canopy reflectance. Due to the large coverage of the airborne LiDAR data with high point density (average 30 points $/ \mathrm{m}^{2}$ ), the canopy cover of the park could be retrieved with a good accuracy. Once modeled and known for each pixel, the canopy cover influence on the variation of canopy reflectance was effectively minimized, which was the key to accurately estimating LWC through model inversion. Another factor that contributed to the variation of canopy reflectance was the background reflectance. In this study, for the first time, the background reflectance at the regional scale was acquired through the combination of LiDAR and hyperspectral data. A LiDAR-derived canopy height model (CHM) was used as a mask to extract the background reflectance in the 
hyperspectral imagery. A threshold of $0.1 \mathrm{~m}$ effectively masked (eliminated) those pixels under the influence of understory vegetation. Allowing the soil reflectance to change within the range of remote sensing derived spectra in the LUT generation could better map the variation of the real background than using a standard (single) soil reflectance.

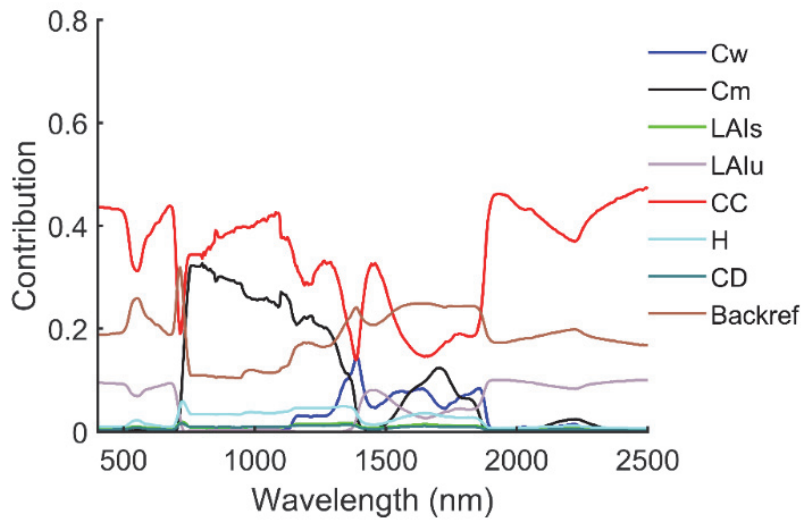

Figure 6.8 Results of FAST first-order sensitivity coefficients to canopy reflectance with modified INFORM (Cw: leaf water content, $\mathrm{Cm}$ : dry mater content, LAIs: single tree LAI, LAIu: understory LAI, CC: canopy cover, H: height, CD: crown diameter, Backref: background reflectance)

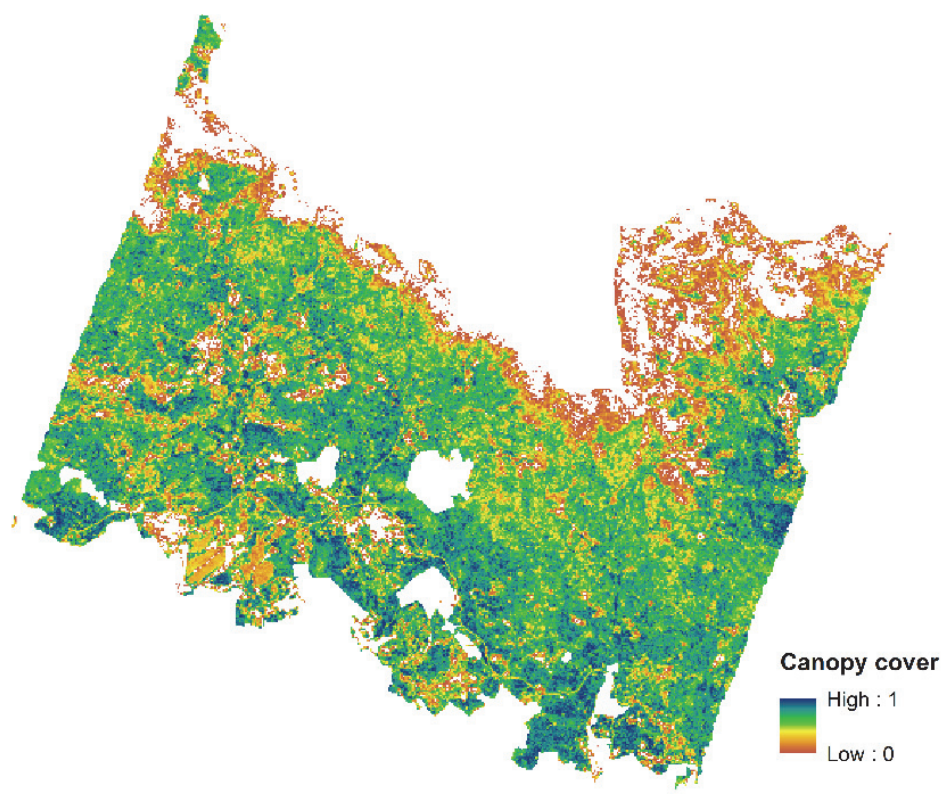

Figure 6.9 Canopy cover mapped using LiDAR data in the Bavarian Forest National Park, Germany

The results show that the introduction of a priori soil reflectance, vegetation cover and understory LAI information obtained from LiDAR significantly improved the accuracy 
of LWC retrieval when inverting INFORM, from an $R^{2}$ of 0.55 and a normalized RMSE (nRMSE) of 0.28 to 0.78 and 0.19 , respectively. The spatial variation of LWC was significantly in accordance with the distribution of broadleaf, needle leaf and mixed forests (Table 6.2, Fig. 6.10), which appeared to be consistent with ecological knowledge. Needle leaf forests had a higher LWC than broadleaf forests. Hatcher (1990) showed that conifers had greater concentrations of all measured foliar constituents than broadleaf trees. As can be seen in Fig. 6.10 (a), striping has appeared in the LWC map. This is due to the edge of the flying strips of the hyperspectral data.

Table 6.2 Summary of statistics for leaf water content of different forest types

\begin{tabular}{lccc}
\hline Forest type & Needle leaf & Broadleaf & Mixed \\
Mean $\left(\mathrm{g} / \mathrm{cm}^{2}\right)$ & 0.021 & 0.016 & 0.019 \\
\hline
\end{tabular}


(a)

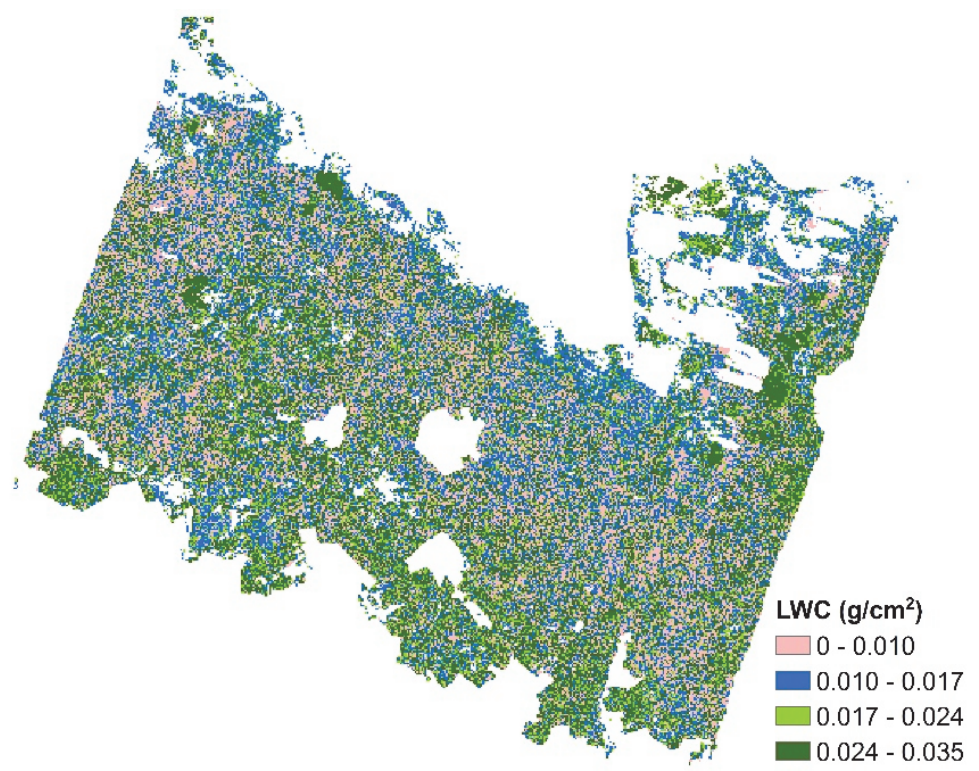

(b)

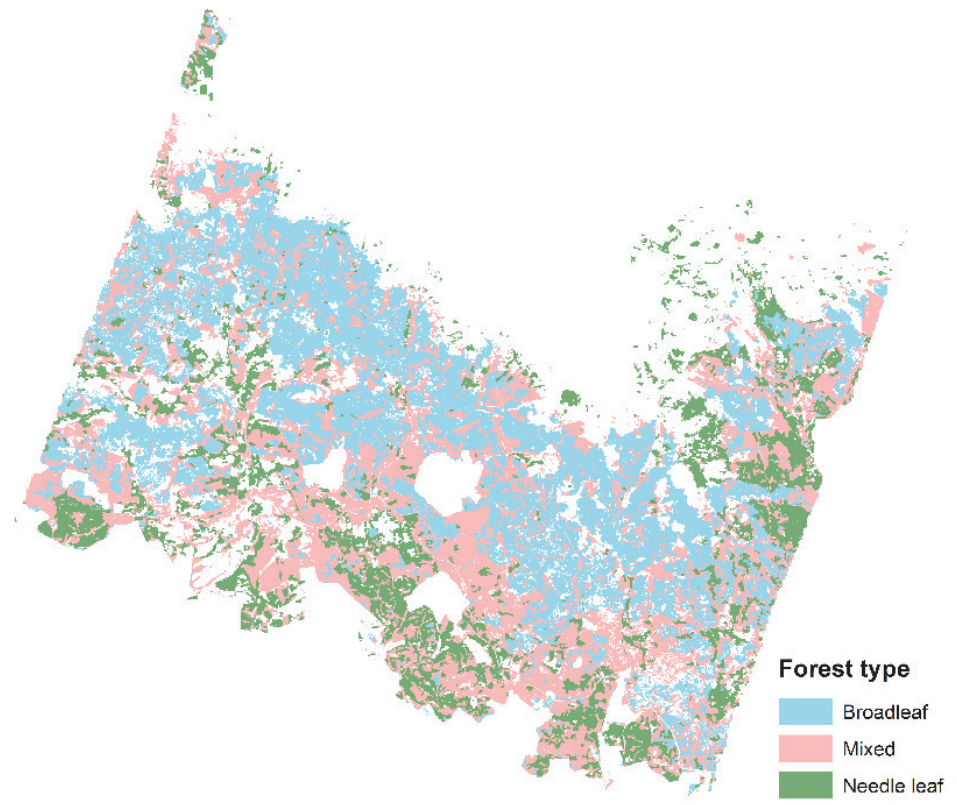

Figure 6.10 Leaf water content mapping (a) and forest type map (b) 


\subsection{Foliar and woody materials discriminated using terrestrial LiDAR}

Quantitative characterization of the forest structure, especially the separation of foliar and woody materials, is critical to accurately estimate biophysical variables (e.g. leaf area index) as well as biochemical variables such as LWC (Ma et al. 2016b). TLS with high point density and small beam size permits an accurate characterization of forest structure (Zheng et al. 2016a). The geometric-based classification has been applied to the separation of foliar and woody materials (Ma et al. 2016a; Zheng et al. 2016b), demonstrating that the local dimensionality features played a significant role in differentiating leaves and stems as they showed different geometric features. Radiometric information has also been used for the classification of these two components since foliar and woody materials have different backscatter intensities (Béland et al. 2011). The combination of geometric and radiometric features for the separation of foliar and woody materials is yet to be explored.

The geometric and radiometric features of a point are calculated based on a group of local neighborhood points. The local neighborhood is defined as a spherical shape (Demantke et al. 2011), where local points are within a fixed radius (Koenig et al. 2015). So the features of each point can be calculated within this local neighborhood. However, the size of the neighborhood is difficult to determine. Different sizes can result in variation in features, thus affecting the classification accuracy.

Chapter 5 evaluated an adaptive radius near-neighbor search algorithm to obtain both geometric and radiometric features for the separation of foliar, and woody and ground materials from TLS data. The combination of radiometric and geometric features yielded a more accurate classification result. Fig. 6.11 showed the clear division between these three classes. Figure 6.12 demonstrates that the combination of geometric and radiometric features produced better classification results than using either of them alone. In the geometric domain, wood could be separated from the ground and foliage, while broadleaf species had a similar surface feature to the ground. Intensity provided a good way to separate the foliage from the other two classes due to the water absorption in leaves. 


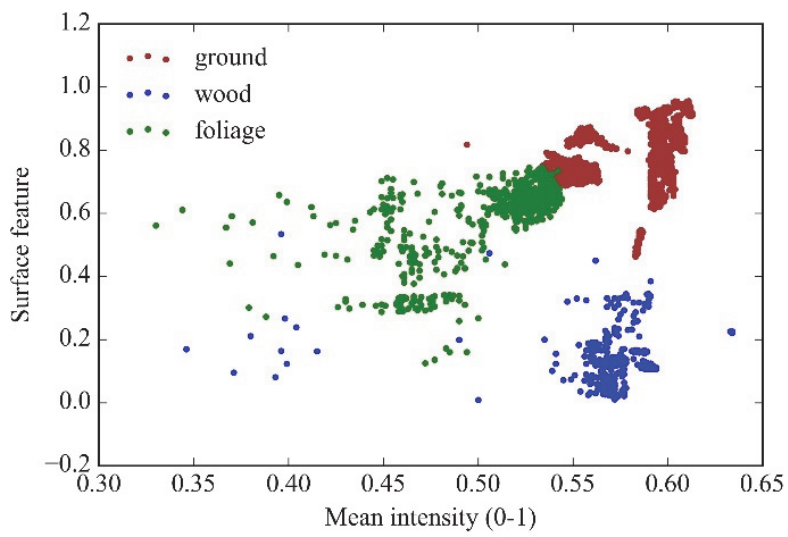

Figure 6.11 The distribution of the ground, wood, and foliage in the geometric (surface feature: the shape of the local points is close to a surface) and radiometric domain.

(a)

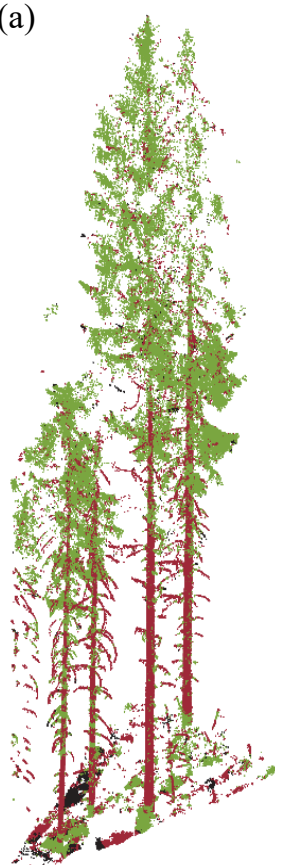

foliage points (b)

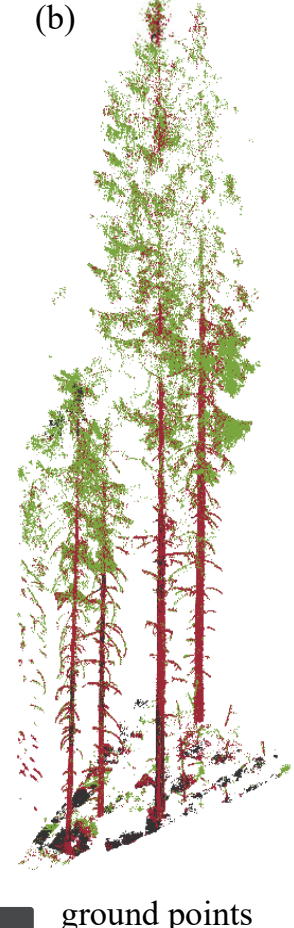

(c)

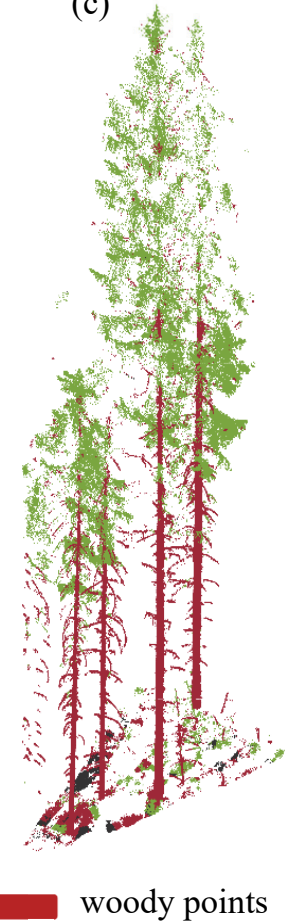

Figure 6.12 Visual comparison of classification results based on (a) radiometric features, (b) geometric features, and (c) the combination of both radiometric and geometric features

The results of the classification also demonstrated that the adaptive radius near-neighbor search performs with a higher accuracy when compared to the fixed radius near-neighbor search in the classification between foliage, wood, and ground (Table 6.3). The highest 
accuracy was achieved by using the adaptive radius, achieving an average overall accuracy of $84.4 \%$. An important factor appears to be that the dimensionality of a local cluster of points varies as the search radius changes (Brodu and Lague 2012). At the scale of a few centimeters, the stem could appear as a surface feature (2D), whereas at a large scale, it is more likely to be a linear feature (1D). The adaptive radius search method chooses the optimal size of the radius. The choice of using three radii of $0.2 \mathrm{~m}, 0.3 \mathrm{~m}$, and $0.4 \mathrm{~m}$ was based on the reality of the plots and a tradeoff between computational efficiency and accuracy. To achieve a more accurate result, a large number of radii is recommended. In the meantime, the size of the radii should be determined based the stem size of the study area. Fig. 6.13 shows the final results of the classification of a broadleaf plot and a needle leaf plot, indicating that the method used in Chapter 5 has the potential to be applied in different forest types.

Table 6.3 Average accuracy of the classification results using different searching radius

\begin{tabular}{|c|c|c|c|c|c|c|c|}
\hline \multicolumn{2}{|c|}{ Adaptive radius } & \multicolumn{2}{|c|}{ Radius: $0.2 \mathrm{~m}$} & \multicolumn{2}{|c|}{ Radius: $0.3 \mathrm{~m}$} & \multicolumn{2}{|c|}{ Radius: $0.4 \mathrm{~m}$} \\
\hline $\begin{array}{c}\text { Overall } \\
\text { accuracy } \\
(\%)\end{array}$ & Kappa & $\begin{array}{c}\text { Overall } \\
\text { accuracy } \\
(\%)\end{array}$ & Kappa & $\begin{array}{c}\text { Overall } \\
\text { accuracy } \\
(\%)\end{array}$ & $\begin{array}{c}\text { Kapp } \\
\text { a }\end{array}$ & $\begin{array}{c}\text { Overall } \\
\text { accuracy } \\
(\%)\end{array}$ & $\begin{array}{c}\text { Kapp } \\
\text { a }\end{array}$ \\
\hline 84.4 & 0.75 & 72.4 & 0.58 & 69.0 & 0.51 & 70.0 & 0.54 \\
\hline
\end{tabular}

(a)

(b)
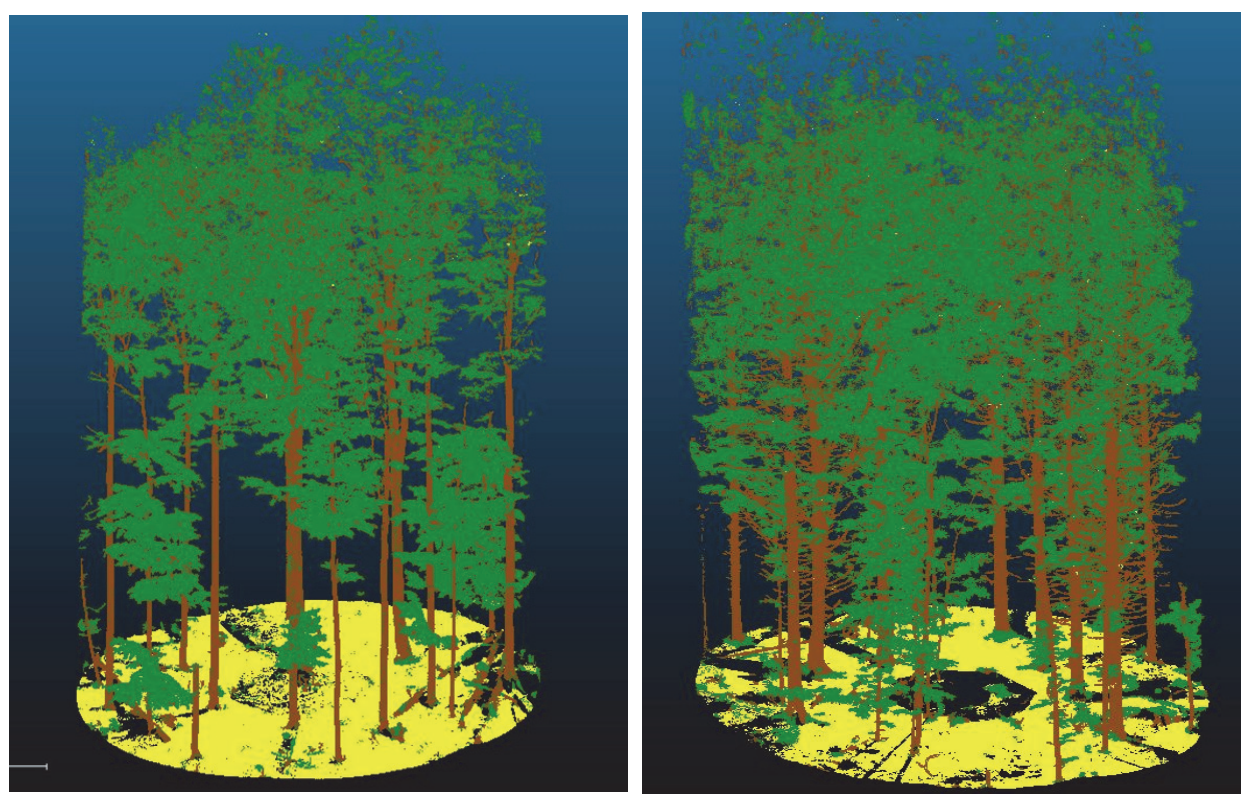

Figure 6.13 Classification results for a broadleaf plot and a needle leaf plot. (a) broadleaf, (b) needle leaf. 


\subsection{Broader implication of leaf water content estimation for ecological studies}

This thesis explored the potential of LiDAR remote sensing alone for LWC estimation at the leaf and individual canopy scales, and the combination of LiDAR and hyperspectral data for LWC estimation at the regional scale. The results indicate that the LWC distribution within individual leaves and the vertical distribution of LWC within vegetation canopy can be estimated using terrestrial LiDAR, and the prior information derived from LiDAR and hyperspectral data can significantly improve the estimation of LWC through the inversion of radiative transfer models at the regional scale. Using the calibration methods developed in this thesis, the approaches have the potential to be transferred to other sites.

At the leaf and individual canopy scale, the distribution of LWC within leaves and an individual canopy estimated from terrestrial LiDAR can provide useful information for forest management. Especially the estimation of the differences of LWC at different height of a plant might be helpful to determine whether a plant is under stress conditions such as drought. At the regional scale, the accurate estimation of LWC is critical for forest managements. Forests provide a variety of goods and ecosystem services for humans, including the habitat for plant and animal species, carbon storage for climate change mitigation and adaptation, water supply for industries and communities, timber for wood products and ecotourism (Chornesky et al. 2015). The variation of LWC could significantly affect forest fire susceptibility and severity, thus the ecosystem services. Regional and global mapping of LWC can further our understanding of physiological processes and ecosystem functions such as photosynthesis, transpiration, thermal regulation, net primary production process and forest fire susceptibility and propagation (Chuvieco et al. 2004; Sanchez et al. 1983; Scriber 1977b). In addition, it could improve the predictions of how forests will change in the future, which can be useful for environmental conservation and management and resource policy making (Asner et al. 2016).

This study is focused on the estimation of LWC over the forest ecosystem. However, the method is in principle transferable to other types of ecosystems such as agricultural and grassland ecosystems. The knowledge of LWC can provide useful information in agriculture for irrigation decisions and drought assessment (Colombo et al. 2008). Grasslands by nature are prone to fires, so improving the estimation of LWC is of importance to assess fire susceptibility and propagation (Dasgupta et al. 2009). The relatively homogenous nature of agriculture and grass landscapes is expected to limit the confounding contribution of biophysical factors to canopy reflectance (Dasgupta et al. 2009). However, canopy reflectance over agriculture and grass landscape can be affected by the background, especially in areas with open canopies. The combination of LiDAR 
and hyperspectral data offers the means to obtain canopy cover and background reflectance information, so the confounding effects can be largely ruled out.

\subsection{Future work and recommendations}

The wavelength of the TLS is $1550 \mathrm{~nm}$ falling in the water absorption region. There are a few other existing instruments with different wavelengths which have been used for the estimation of plant biochemical variables. For example, the Leica ScanStaion 2 employs a pulsed green $(532 \mathrm{~nm})$ laser that is advantageous for the estimation of chlorophyll concentration (Eitel et al. 2010a). The same TLS has also been used for the evaluation of nitrogen status (Eitel et al. 2011a). Magney et al. (2014) demonstrated that the green laser return intensity was significantly correlated with non-photochemical quenching. Although the usage of the single wavelength TLS for plant biochemical variables is still limited, these studies show that TLS holds promise to provide detailed information about plant physiological status as long as the incidence angle effect can be tackled.

The instrument in the aforementioned studies employs a single wavelength laser, so the calibration for the distance and incidence angle effects have to be addressed. Nonetheless, recent advances in TLS provided an alternative to the calibration procedure: multiwavelength laser scanning. With dual-bands, even multiple bands, TLS offers the potential to obtain vegetation indices. The normalized ratio of two selected wavelengths should be insensitive to the incidence angle as the influence can be canceled when the intensity of both wavelengths is similarly affected by the incidence angle (Gaulton et al. 2013). However, the selection of wavelengths has to be careful. A recent study showed that the laser spectral indices based on ratio combinations of green and red laser return intensity reduced the distance effect, while they did not account for variations in leaf angle (Eitel et al. 2014), suggesting the pronounced differences in the bidirectional reflectance distribution factor between the green and red laser wavelengths at the leaf scale. In another study, a number of spectral indices based on the near and middle infrared (1063 nm and $1545 \mathrm{~nm}$ ) were tested for LWC estimation (Gaulton et al. 2013). A strong relationship $\left(R^{2}=0.87\right)$ was found between a normalized ratio and measured LWC, indicating that not only the incidence angle effect could be canceled, the partial beam hits effect could also be minimized by using a dual-wavelength laser scanning system. The development of these new instruments will not only benefit the estimation of plant biochemical variables, but also biophysical variables and tree species classification.

At the regional scale, airborne LiDAR and hyperspectral data were combined to alleviate the ill-posed problem when inverting RTMs. Canopy cover and understory LAI was obtained from a LiDAR metric, and background reflectance was derived from LiDAR and hyperspectral data. LiDAR has the potential to obtain additional structural variables other than canopy cover such canopy height profile (Fieber et al. 2015) and to classify tree species (Yu et al. 2017). These canopy structural parameters may be input to more 
complicated radiative transfer models (Bye et al. 2017; Gastellu-Etchegorry et al. 2015; North et al. 2010) as prior information in model inversion for not only LWC content but also other parameters estimation. Moreover, the recently developed multispectral laser scanning techniques is an attractive option for forest studies, as it not only provides 3D point clouds but also multispectral information (St-Onge and Budei 2015; Yu et al. 2017). This technique can avoid the uncertainty from the geometric and radiometric registration between two different datasets (Wallace et al. 2012). The multispectral information may also tackle many problems relating to the calibration of the intensity data. Coupling with the geometric information, multispectral LiDAR offers a powerful tool for forest canopy radiative transfer modeling, thus the estimation of forest essential biodiversity variables.

The method of combining LiDAR and hyperspectral data in an RTM could potentially be applied to spaceborne data (in the future) for global LWC as well as other EBVs mapping. The main limitation of current available passive satellite data is the spectral or/and spatial resolution. The open satellite data such as Landsat and Sentinel data do not provide detailed narrow spectral information, so the spectral characteristics of plant traits are often masked by convolving the incoming radiation across broad wavelength ranges (Broge and Leblanc 2001). The confounding effects of vegetation structure and forest background are more difficult to eliminate. The integration of LiDAR is of key importance for this matter. In terms of the resolution of available spaceborne LiDAR data, the Geoscience Laser Altimeter System (GLAS) sensor has a large footprint $(70 \mathrm{~m}$ diameter) with a density of 0.52 points $/ \mathrm{km}^{2}$ which is not enough for detailed mapping of EBVs. The Global Ecosystem Dynamics Investigation (GEDI) LiDAR will be launched in 2019, which produces the first high resolution (footprint $=20 \mathrm{~m}$ diameter) laser ranging observations of the 3D structure of the Earth (Lidar 2017). It provides a great opportunity for monitoring global EBVs at a relatively high resolution. 


\section{Bibliography}

Alexander, C., Tansey, K., Kaduk, J., Holland, D., \& Tate, N.J. (2010). Backscatter coefficient as an attribute for the classification of full-waveform airborne laser scanning data in urban areas. ISPRS Journal of Photogrammetry and Remote Sensing, 65, 423-432

Ali, A.M., Darvishzadeh, R., Skidmore, A.K., \& Duren, I.v. (2016a). Effects of canopy structural variables on retrieval of leaf dry matter content and specific leaf area from remotely sensed data. IEEE Journal of Selected Topics in Applied Earth Observations and Remote Sensing, 9, 898-909

Ali, A.M., Darvishzadeh, R., Skidmore, A.K., \& van Duren, I. (2016b). Effects of canopy structural variables on retrieval of leaf dry matter content and specific leaf area from remotely sensed data. IEEE Journal of Selected Topics in Applied Earth Observations and Remote Sensing, 9, 898-909

Ali, A.M., Darvishzadeh, R., Skidmore, A.K., \& van Duren, I. (2017). Specific leaf area estimation from leaf and canopy reflectance through optimization and validation of vegetation indices. Agricultural and Forest Meteorology, 236, 162-174

Ali, A.M., Darvishzadeh, R., Skidmore, A.K., van Duren, I., Heiden, U., \& Heurich, M. (2016c). Estimating leaf functional traits by inversion of PROSPECT: Assessing leaf dry matter content and specific leaf area in mixed mountainous forest. International Journal of Applied Earth Observation and Geoinformation, 45, 6676

Anderson, H.W., Hoover, M.D., \& Reinhart, K.G. (1976). Forests and water: effects of forest management on floods, sedimentation, and water supply

Anderson, J.E., Plourde, L.C., Martin, M.E., Braswell, B.H., Smith, M.-L., Dubayah, R.O., Hofton, M.A., \& Blair, J.B. (2008). Integrating waveform lidar with hyperspectral imagery for inventory of a northern temperate forest. Remote Sensing of Environment, 112, 1856-1870

Asner, G.P., Brodrick, P.G., Anderson, C.B., Vaughn, N., Knapp, D.E., \& Martin, R.E. (2016). Progressive forest canopy water loss during the 2012-2015 California drought. Proceedings of the National Academy of Sciences, 113, E249-E255

Asner, G.P., \& Martin, R.E. (2008). Spectral and chemical analysis of tropical forests: Scaling from leaf to canopy levels. Remote Sensing of Environment, 112, 39583970

Asner, G.P., Martin, R.E., Anderson, C.B., \& Knapp, D.E. (2015). Quantifying forest canopy traits: Imaging spectroscopy versus field survey. Remote Sensing of Environment, 158, 15-27

Asner, G.P., Martin, R.E., Knapp, D.E., Tupayachi, R., Anderson, C., Carranza, L., Martinez, P., Houcheime, M., Sinca, F., \& Weiss, P. (2011). Spectroscopy of canopy chemicals in humid tropical forests. Remote Sensing of Environment, 115, 3587-3598

Atzberger, C. (2000). Development of an invertible forest reflectance model: The INFOR-Model. In, A decade of trans-European remote sensing cooperation. Proceedings of the 20th EARSeL Symposium Dresden, Germany (pp. 39-44)

Atzberger, C., Guérif, M., Baret, F., \& Werner, W. (2010). Comparative analysis of three chemometric techniques for the spectroradiometric assessment of canopy chlorophyll content in winter wheat. Computers and Electronics in Agriculture, $73,165-173$ 
Beckmann, P., \& Spizzichino, A. (1987). The scattering of electromagnetic waves from rough surfaces. New York: Artech Print on Demand

Béland, M., Baldocchi, D.D., Widlowski, J.-L., Fournier, R.A., \& Verstraete, M.M. (2014). On seeing the wood from the leaves and the role of voxel size in determining leaf area distribution of forests with terrestrial LiDAR. Agricultural and Forest Meteorology, 184, 82-97

Béland, M., Widlowski, J.-L., Fournier, R.A., Côté, J.-F., \& Verstraete, M.M. (2011). Estimating leaf area distribution in savanna trees from terrestrial LiDAR measurements. Agricultural and Forest Meteorology, 151, 1252-1266

Belgiu, M., \& Drăguț, L. (2016). Random forest in remote sensing: A review of applications and future directions. ISPRS Journal of Photogrammetry and Remote Sensing, 114, 24-31

Bigdeli, B., Samadzadegan, F., \& Reinartz, P. (2015). Fusion of hyperspectral and LIDAR data using decision template-based fuzzy multiple classifier system. International Journal of Applied Earth Observation and Geoinformation, 38, 309320

Bowman, W.D. (1989). The relationship between leaf water status, gas exchange, and spectral reflectance in cotton leaves. Remote Sensing of Environment, 30, 249-255

Bowyer, P., \& Danson, F.M. (2004). Sensitivity of spectral reflectance to variation in live fuel moisture content at leaf and canopy level. Remote Sensing of Environment, 92, 297-308

Briese, C., Höfle, B., Lehner, H., Wagner, W., Pfennigbauer, M., \& Ullrich, A. (2008). Calibration of full-waveform airborne laser scanning data for object classification. In, SPIE Defense and Security Symposium (pp. 1-8): International Society for Optics and Photonics

Brodu, N., \& Lague, D. (2012). 3D terrestrial lidar data classification of complex natural scenes using a multi-scale dimensionality criterion: Applications in geomorphology. ISPRS Journal of Photogrammetry and Remote Sensing, 68, 121 134

Broge, N.H., \& Leblanc, E. (2001). Comparing prediction power and stability of broadband and hyperspectral vegetation indices for estimation of green leaf area index and canopy chlorophyll density. Remote Sensing of Environment, 76, 156172

Bunnell, F.L., \& Vales, D.J. (1990). Comparison of methods for estimating forest overstory cover: differences among techniques. Canadian Journal of Forest Research, 20, 101-107

Bye, I.J., North, P.R.J., Los, S.O., Kljun, N., Rosette, J.A.B., Hopkinson, C., Chasmer, L., \& Mahoney, C. (2017). Estimating forest canopy parameters from satellite waveform LiDAR by inversion of the FLIGHT three-dimensional radiative transfer model. Remote Sensing of Environment, 188, 177-189

Caetano, M.R., \& Pereira, J.M.T. (1996). Effect of the understory on the estimation of coniferous forest leaf area index (LAI) based on remotely sensed data. In (pp. 6371)

Calders, K., Armston, J., Newnham, G., Herold, M., \& Goodwin, N. (2014). Implications of sensor configuration and topography on vertical plant profiles derived from terrestrial LiDAR. Agricultural and Forest Meteorology, 194, 104-117

Cao, C., Bao, Y., Xu, M., Chen, W., Zhang, H., He, Q., Li, Z., Guo, H., Li, J., Li, X., \& Li, G. (2012). Retrieval of forest canopy attributes based on a geometric-optical 
model using airborne LiDAR and optical remote-sensing data. International Journal of Remote Sensing, 33, 692-709

Cao, L., Coops, N.C., Innes, J.L., Dai, J., Ruan, H., \& She, G. (2016). Tree species classification in subtropical forests using small-footprint full-waveform LiDAR data. International Journal of Applied Earth Observation and Geoinformation, 49, 39-51

Ceccato, P., Flasse, S., Tarantola, S., Jacquemoud, S., \& Grégoire, J.-M. (2001). Detecting vegetation leaf water content using reflectance in the optical domain. Remote Sensing of Environment, 77, 22-33

Ceccato, P., Gobron, N., Flasse, S., Pinty, B., \& Tarantola, S. (2002). Designing a spectral index to estimate vegetation water content from remote sensing data: Part 1: Theoretical approach. Remote Sensing of Environment, 82, 188-197

Champagne, C.M., Staenz, K., Bannari, A., McNairn, H., \& Deguise, J.-C. (2003). Validation of a hyperspectral curve-fitting model for the estimation of plant water content of agricultural canopies. Remote Sensing of Environment, 87, 148-160

Chavana-Bryant, C., Malhi, Y., Wu, J., Asner, G.P., Anastasiou, A., Enquist, B.J., Caravasi, C., Eric, G., Doughty, C.E., \& Saleska, S.R. (2016). Leaf aging of Amazonian canopy trees as revealed by spectral and physiochemical measurements. New Phytologist, 214, 1049-1063

Chen, J.M., \& Cihlar, J. (1995). Quantifying the effect of canopy architecture on optical measurements of leaf area index using two gap size analysis methods. IEEE Transactions on Geoscience and Remote Sensing, 33, 777-787

Cheng, T., Rivard, B., \& Sánchez-Azofeifa, A. (2011). Spectroscopic determination of leaf water content using continuous wavelet analysis. Remote Sensing of Environment, 115, 659-670

Cheng, T., Rivard, B., Sánchez-Azofeifa, A.G., Féret, J.-B., Jacquemoud, S., \& Ustin, S.L. (2012). Predicting leaf gravimetric water content from foliar reflectance across a range of plant species using continuous wavelet analysis. Journal of Plant Physiology, 169, 1134-1142

Cheng, Y.-B., Zarco-Tejada, P.J., Riaño, D., Rueda, C.A., \& Ustin, S.L. (2006). Estimating vegetation water content with hyperspectral data for different canopy scenarios: Relationships between AVIRIS and MODIS indexes. Remote Sensing of Environment, 105, 354-366

Chi-Keung, T., \& Medioni, G. (2002). Curvature-augmented tensor voting for shape inference from noisy 3D data. IEEE Transactions on Pattern Analysis and Machine Intelligence, 24, 858-864

Chornesky, E.A., Ackerly, D.D., Beier, P., Davis, F.W., Flint, L.E., Lawler, J.J., Moyle, P.B., Moritz, M.A., Scoonover, M., \& Byrd, K. (2015). Adapting California's ecosystems to a changing climate. Bioscience, 65, 247-262

Chuvieco, E., Cocero, D., Riaño, D., Martin, P., Martínez-Vega, J., de la Riva, J., \& Pérez, F. (2004). Combining NDVI and surface temperature for the estimation of live fuel moisture content in forest fire danger rating. Remote Sensing of Environment, 92, 322-331

Chuvieco, E., \& Kasischke, E.S. (2007). Remote sensing information for fire management and fire effects assessment. Journal of Geophysical Research: Biogeosciences, 112, n/a-n/a

Chuvieco, E., Riano, D., Aguado, I., \& Cocero, D. (2002). Estimation of fuel moisture content from multitemporal analysis of Landsat Thematic Mapper reflectance 
data: applications in fire danger assessment. International Journal of Remote Sensing, 23, 2145-2162

Ciganda, V., Gitelson, A., \& Schepers, J. (2008). Vertical profile and temporal variation of chlorophyll in maize canopy: Quantitative "crop vigor" indicator by means of reflectance-based techniques. Agronomy Journal, 100, 1409-1417

Clawges, R., Vierling, L., Calhoon, M., \& Toomey, M. (2007). Use of a ground-based scanning lidar for estimation of biophysical properties of western larch (Larix occidentalis). International Journal of Remote Sensing, 28, 4331-4344

Colombo, R., Meroni, M., Marchesi, A., Busetto, L., Rossini, M., Giardino, C., \& Panigada, C. (2008). Estimation of leaf and canopy water content in poplar plantations by means of hyperspectral indices and inverse modeling. Remote Sensing of Environment, 112, 1820-1834

Combal, B., Baret, F., \& Weiss, M. (2002). Improving canopy variables estimation from remote sensing data by exploiting ancillary information. Case study on sugar beet canopies. Agronomie, 22, 205-215

Combal, B., Baret, F., Weiss, M., Trubuil, A., Macé, D., Pragnère, A., Myneni, R., Knyazikhin, Y., \& Wang, L. (2003). Retrieval of canopy biophysical variables from bidirectional reflectance: Using prior information to solve the ill-posed inverse problem. Remote Sensing of Environment, 84, 1-15

Coren, F., \& Sterzai, P. (2006). Radiometric correction in laser scanning. International Journal of Remote Sensing, 27, 3097-3104

Côté, J.-F., Fournier, R.A., Frazer, G.W., \& Olaf Niemann, K. (2012). A fine-scale architectural model of trees to enhance LiDAR-derived measurements of forest canopy structure. Agricultural and Forest Meteorology, 166, 72-85

Côté, J.-F., Widlowski, J.-L., Fournier, R.A., \& Verstraete, M.M. (2009). The structural and radiative consistency of three-dimensional tree reconstructions from terrestrial lidar. Remote Sensing of Environment, 113, 1067-1081

Cruz, M.G., Alexander, M.E., \& Wakimoto, R.H. (2004). Modeling the likelihood of crown fire occurrence in conifer forest stands. Forest Science, 50, 640-658

Curran, P., \& Williamson, H. (1986). Sample size for ground and remotely sensed data. Remote Sensing of Environment, 20, 31-41

Danson, F.M., Gaulton, R., Armitage, R.P., Disney, M., Gunawan, O., Lewis, P., Pearson, G., \& Ramirez, A.F. (2014). Developing a dual-wavelength full-waveform terrestrial laser scanner to characterize forest canopy structure. Agricultural and Forest Meteorology, 198-199, 7-14

Danson, F.M., Steven, M.D., Malthus, T.J., \& Clark, J.A. (1992). High-spectral resolution data for determining leaf water content. International Journal of Remote Sensing, $13,461-470$

Darvishzadeh, R., Atzberger, C., Skidmore, A., \& Schlerf, M. (2011). Mapping grassland leaf area index with airborne hyperspectral imagery: A comparison study of statistical approaches and inversion of radiative transfer models. ISPRS Journal of Photogrammetry and Remote Sensing, 66, 894-906

Darvishzadeh, R., Matkan, A.A., \& Ahangar, A.D. (2012). Inversion of a radiative transfer model for estimation of rice canopy chlorophyll content using a lookuptable approach. IEEE Journal of Selected Topics in Applied Earth Observations and Remote Sensing, 5, 1222-1230

Darvishzadeh, R., Skidmore, A., Atzberger, C., \& van Wieren, S. (2008a). Estimation of vegetation LAI from hyperspectral reflectance data: Effects of soil type and plant 
architecture. International Journal of Applied Earth Observation and Geoinformation, 10, 358-373

Darvishzadeh, R., Skidmore, A., Schlerf, M., \& Atzberger, C. (2008b). Inversion of a radiative transfer model for estimating vegetation LAI and chlorophyll in a heterogeneous grassland. Remote Sensing of Environment, 112, 2592-2604

Dasgupta, S., Qu, J.J., \& Bhoi, S. (2009). Constrained radiative transfer inversions for vegetation moisture retrievals in grasslands. Journal of Applied Remote Sensing, 3, 031503

Davidson, A., Wang, S., \& Wilmshurst, J. (2006). Remote sensing of grasslandshrubland vegetation water content in the shortwave domain. International Journal of Applied Earth Observation and Geoinformation, 8, 225-236

Dawson, T., Curran, P., North, P., \& Plummer, S. (1999). The propagation of foliar biochemical absorption features in forest canopy reflectance: A theoretical analysis. Remote Sensing of Environment, 67, 147-159

Dawson, T.P., Curran, P.J., \& Plummer, S.E. (1998). LIBERTY-Modeling the effects of leaf biochemical concentration on reflectance spectra. Remote Sensing of Environment, 65, 50-60

de Jong, S.M., Addink, E.A., \& Doelman, J.C. (2014). Detecting leaf-water content in Mediterranean trees using high-resolution spectrometry. International Journal of Applied Earth Observation and Geoinformation, 27, Part B, 128-136

de Jong, S.M., Addink, E.A., Hoogenboom, P., \& Nijland, W. (2012). The spectral response of Buxus sempervirens to different types of environmental stress-A laboratory experiment. ISPRS Journal of Photogrammetry and Remote Sensing, $74,56-65$

de Leeuw, J., Jia, H., Yang, L., Liu, X., Schmidt, K., \& Skidmore, A. (2006). Comparing accuracy assessments to infer superiority of image classification methods. International Journal of Remote Sensing, 27, 223-232

Demantke, J., Mallet, C., David, N., \& Vallet, B. (2011). Dimensionality based scale selection in 3D lidar point clouds. The International Archives of the Photogrammetry, Remote Sensing and Spatial Information Sciences, 38, W12

Ding, Q., Chen, W., King, B., Liu, Y., \& Liu, G. (2013). Combination of overlap-driven adjustment and Phong model for LiDAR intensity correction. ISPRS Journal of Photogrammetry and Remote Sensing, 75, 40-47

Eitel, J.U., Vierling, L.A., \& Long, D.S. (2010a). Simultaneous measurements of plant structure and chlorophyll content in broadleaf saplings with a terrestrial laser scanner. Remote Sensing of Environment, 114, 2229-2237

Eitel, J.U., Vierling, L.A., Long, D.S., \& Hunt, E.R. (2011a). Early season remote sensing of wheat nitrogen status using a green scanning laser. Agricultural and Forest Meteorology, 151, 1338-1345

Eitel, J.U.H., Magney, T.S., Vierling, L.A., \& Dittmar, G. (2014). Assessment of crop foliar nitrogen using a novel dual-wavelength laser system and implications for conducting laser-based plant physiology. ISPRS Journal of Photogrammetry and Remote Sensing, 97, 229-240

Eitel, J.U.H., Vierling, L.A., \& Long, D.S. (2010b). Simultaneous measurements of plant structure and chlorophyll content in broadleaf saplings with a terrestrial laser scanner. Remote Sensing of Environment, 114, 2229-2237 
Eitel, J.U.H., Vierling, L.A., Long, D.S., \& Hunt, E.R. (2011b). Early season remote sensing of wheat nitrogen status using a green scanning laser. Agricultural and Forest Meteorology, 151, 1338-1345

Eriksson, H.M., Eklundh, L., Kuusk, A., \& Nilson, T. (2006). Impact of understory vegetation on forest canopy reflectance and remotely sensed LAI estimates. Remote Sensing of Environment, 103, 408-418

EVANS, B.H. (1932). The physiology of succulent plants. Biological Reviews, 7, 181211

FAO (2017). Second expert meeting on harmonizing forest-related definitions for use by various stakeholders. In

Fieber, K.D., Davenport, I.J., Ferryman, J.M., Gurney, R.J., Walker, J.P., \& Hacker, J.M. (2013). Analysis of full-waveform LiDAR data for classification of an orange orchard scene. ISPRS Journal of Photogrammetry and Remote Sensing, 82, 63-82

Fieber, K.D., Davenport, I.J., Tanase, M.A., Ferryman, J.M., Gurney, R.J., Becerra, V.M., Walker, J.P., \& Hacker, J.M. (2015). Validation of Canopy Height Profile methodology for small-footprint full-waveform airborne LiDAR data in a discontinuous canopy environment. ISPRS Journal of Photogrammetry and Remote Sensing, 104, 144-157

Fleming, M.D. (1988). An integrated approach for automated cover-type mapping of large inaccessible areas in Alaska. Photogrammetric Engineering and Remote Sensing, 54, 357-362

Fourty, T., \& Baret, F. (1998). On spectral estimates of fresh leaf biochemistry. International Journal of Remote Sensing, 19, 1283-1297

Franceschi, M., Teza, G., Preto, N., Pesci, A., Galgaro, A., \& Girardi, S. (2009). Discrimination between marls and limestones using intensity data from terrestrial laser scanner. ISPRS Journal of Photogrammetry and Remote Sensing, 64, 522528

Fu, Z., Wang, J., Song, J.L., Zhou, H.M., Pang, Y., \& Chen, B.S. (2011). Estimation of forest canopy leaf area index using MODIS, MISR, and LiDAR observations. Journal of Applied Remote Sensing, 5, 053530-053530-053521

Gao, B.-c. (1996). NDWI-A normalized difference water index for remote sensing of vegetation liquid water from space. Remote Sensing of Environment, 58, 257-266

Gao, B.-C., \& Goetzt, A.F.H. (1995). Retrieval of equivalent water thickness and information related to biochemical components of vegetation canopies from AVIRIS data. Remote Sensing of Environment, 52, 155-162

García, M., Danson, F.M., Riano, D., Chuvieco, E., Ramirez, F.A., \& Bandugula, V. (2011). Terrestrial laser scanning to estimate plot-level forest canopy fuel properties. International Journal of Applied Earth Observation and Geoinformation, 13, 636-645

García, M., Riaño, D., Chuvieco, E., \& Danson, F.M. (2010). Estimating biomass carbon stocks for a Mediterranean forest in central Spain using LiDAR height and intensity data. Remote Sensing of Environment, 114, 816-830

Gastellu-Etchegorry, J.-P., Yin, T., Lauret, N., Cajgfinger, T., Gregoire, T., Grau, E., Feret, J.-B., Lopes, M., Guilleux, J., Dedieu, G., Malenovský, Z., Cook, B., Morton, D., Rubio, J., Durrieu, S., Cazanave, G., Martin, E., \& Ristorcelli, T. (2015). Discrete Anisotropic Radiative Transfer (DART 5) for Modeling Airborne and Satellite Spectroradiometer and LIDAR Acquisitions of Natural and Urban Landscapes. Remote Sensing, 7, 1667 
Gaulton, R., Danson, F.M., Ramirez, F.A., \& Gunawan, O. (2013). The potential of dualwavelength laser scanning for estimating vegetation moisture content. Remote Sensing of Environment, 132, 32-39

Goetz, A.F. (2009). Three decades of hyperspectral remote sensing of the Earth: A personal view. Remote Sensing of Environment, 113, S5-S16

Gong, P., Pu, R., Biging, G.S., \& Larrieu, M.R. (2003). Estimation of forest leaf area index using vegetation indices derived from Hyperion hyperspectral data. Geoscience and Remote Sensing, IEEE Transactions on, 41, 1355-1362

Grant, L. (1987). Diffuse and specular characteristics of leaf reflectance. Remote Sensing of Environment, 22, 309-322

Gross, H., \& Thoennessen, U. (2006). Extraction of lines from laser point clouds. In, Symposium of ISPRS Commission III: Photogrammetric Computer Vision PCV06. International Archives of Photogrammetry, Remote Sensing and Spatial Information Sciences (pp. 86-91)

Guan, H., Li, J., Chapman, M., Deng, F., Ji, Z., \& Yang, X. (2013). Integration of orthoimagery and lidar data for object-based urban thematic mapping using random forests. International Journal of Remote Sensing, 34, 5166-5186

Guang, Z., \& Moskal, L.M. (2012). Computational-Geometry-Based Retrieval of Effective Leaf Area Index Using Terrestrial Laser Scanning. Geoscience and Remote Sensing, IEEE Transactions on, 50, 3958-3969

Hancock, S., Armston, J., Li, Z., Gaulton, R., Lewis, P., Disney, M., Mark Danson, F., Strahler, A., Schaaf, C., Anderson, K., \& Gaston, K.J. (2015). Waveform lidar over vegetation: An evaluation of inversion methods for estimating return energy. Remote Sensing of Environment, 164, 208-224

Hancock, S., Gaulton, R., \& Danson, F.M. (2017). Angular Reflectance of Leaves With a Dual-Wavelength Terrestrial Lidar and Its Implications for Leaf-Bark Separation and Leaf Moisture Estimation. IEEE Transactions on Geoscience and Remote Sensing, 55, 3084-3090

Hartzell, P.J., Glennie, C.L., \& Finnegan, D.C. (2015). Empirical waveform decomposition and radiometric calibration of a terrestrial full-waveform laser scanner. IEEE Transactions on Geoscience and Remote Sensing, 53, 162-172

Hatcher, P.E. (1990). Seasonal and age-related variation in the needle quality of five conifer species. Oecologia, 85, 200-212

Heinzel, J., \& Koch, B. (2011). Exploring full-waveform LiDAR parameters for tree species classification. International Journal of Applied Earth Observation and Geoinformation, 13, 152-160

Henning, J.G., \& Radtke, P.J. (2006). Ground-based laser imaging for assessing three dimensional forest canopy structure. Photogrammetric Engineering and Remote Sensing, 72, 1349

Heurich, M., Beudert, B., Rall, H., \& Křenová, Z. (2010). National parks as model regions for interdisciplinary long-term ecological research: The bavarian forest and šumavá national parks underway to transboundary ecosystem research. In F. Müller, C. Baessler, H. Schubert, \& S. Klotz (Eds.), Long-Term Ecological Research: Between Theory and Application (pp. 327-344). Dordrecht: Springer Netherlands

Hilker, T., Coops, N.C., Newnham, G.J., van Leeuwen, M., Wulder, M.A., Stewart, J., \& Culvenor, D.S. (2012). Comparison of terrestrial and airborne LiDAR in 
describing stand structure of a thinned lodgepole pine forest. Journal of Forestry, 110, 97-104

Hilker, T., van Leeuwen, M., Coops, N.C., Wulder, M.A., Newnham, G.J., Jupp, D.L., \& Culvenor, D.S. (2010). Comparing canopy metrics derived from terrestrial and airborne laser scanning in a Douglas-fir dominated forest stand. Trees, 24, 819832

Hodgson, M.E., \& Bresnahan, P. (2004). Accuracy of airborne LiDAR-derived elevation. Photogrammetric Engineering \& Remote Sensing, 70, 331-339

Höfle, B. (2014). Radiometric correction of terrestrial LiDAR point cloud data for individual maize plant detection. IEEE Geoscience and Remote Sensing Letters, $11,94-98$

Höfle, B., Hollaus, M., \& Hagenauer, J. (2012). Urban vegetation detection using radiometrically calibrated small-footprint full-waveform airborne LiDAR data. ISPRS Journal of Photogrammetry and Remote Sensing, 67, 134-147

Höfle, B., \& Pfeifer, N. (2007). Correction of laser scanning intensity data: data and model-driven approaches. ISPRS Journal of Photogrammetry and Remote Sensing, 62, 415-433

Hofton, M.A., Minster, J.B., \& Blair, J.B. (2000). Decomposition of laser altimeter waveforms. IEEE Transactions on Geoscience and Remote Sensing, 38, 19891996

Hollaus, M., Aubrecht, C., Höfle, B., Steinnocher, K., \& Wagner, W. (2011). Roughness mapping on various vertical scales based on full-waveform airborne laser scanning data. Remote Sensing, 3, 503-523

Homolova, L., Malenovský, Z., Clevers, J.G., García-Santos, G., \& Schaepman, M.E. (2013). Review of optical-based remote sensing for plant trait mapping. Ecological Complexity, 15, 1-16

Hopkinson, C., \& Chasmer, L. (2009). Testing LiDAR models of fractional cover across multiple forest ecozones. Remote Sensing of Environment, 113, 275-288

Hoppe, H., DeRose, T., Duchamp, T., McDonald, J., \& Stuetzle, W. (1992). Surface reconstruction from unorganized points. In, Proceedings of the 19th annual conference on Computer graphics and interactive techniques (pp. 71-78): ACM

Hosoi, F., Nakai, Y., \& Omasa, K. (2010). Estimation and Error Analysis of Woody Canopy Leaf Area Density Profiles Using 3-D Airborne and Ground-Based Scanning Lidar Remote-Sensing Techniques. Geoscience and Remote Sensing, IEEE Transactions on, 48, 2215-2223

Hosoi, F., Nakai, Y., \& Omasa, K. (2013). 3-D voxel-based solid modeling of a broadleaved tree for accurate volume estimation using portable scanning lidar. ISPRS Journal of Photogrammetry and Remote Sensing, 82, 41-48

Hosoi, F., \& Omasa, K. (2007). Factors contributing to accuracy in the estimation of the woody canopy leaf area density profile using 3D portable lidar imaging. Journal of Experimental Botany, 58, 3463-3473

Hosoi, F., \& Omasa, K. (2009). Estimating vertical plant area density profile and growth parameters of a wheat canopy at different growth stages using three-dimensional portable lidar imaging. ISPRS Journal of Photogrammetry and Remote Sensing, 64, 151-158

Houborg, R., Anderson, M., \& Daughtry, C. (2009). Utility of an image-based canopy reflectance modeling tool for remote estimation of LAI and leaf chlorophyll content at the field scale. Remote Sensing of Environment, 113, 259-274 
Houborg, R., Soegaard, H., \& Boegh, E. (2007). Combining vegetation index and model inversion methods for the extraction of key vegetation biophysical parameters using Terra and Aqua MODIS reflectance data. Remote Sensing of Environment, $106,39-58$

Huang, H., Link, T., Smith, A., \& Chen, C. (2011). Accuracy of the LiDAR-derived DEM in dense shrub areas in mountainous NW US. In, Proceedings 2011 IEEE International Conference on Spatial Data Mining and Geographical Knowledge Services (pp. 373-377)

Huemmrich, K. (2001). The GeoSail model: a simple addition to the SAIL model to describe discontinuous canopy reflectance. Remote Sensing of Environment, 75, 423-431

Hyyppä, J., Hyyppä, H., Leckie, D., Gougeon, F., Yu, X., \& Maltamo, M. (2008). Review of methods of small-footprint airborne laser scanning for extracting forest inventory data in boreal forests. International Journal of Remote Sensing, 29, 1339-1366

Jackson, T.J., Chen, D., Cosh, M., Li, F., Anderson, M., Walthall, C., Doriaswamy, P., \& Hunt, E.R. (2004). Vegetation water content mapping using Landsat data derived normalized difference water index for corn and soybeans. Remote Sensing of Environment, 92, 475-482

Jacquemoud, S. (1993). Inversion of the PROSPECT + SAIL canopy reflectance model from AVIRIS equivalent spectra: Theoretical study. Remote Sensing of Environment, 44, 281-292

Jacquemoud, S., \& Baret, F. (1990). PROSPECT: A model of leaf optical properties spectra. Remote Sensing of Environment, 34, 75-91

Jacquemoud, S., Baret, F., Andrieu, B., Danson, F.M., \& Jaggard, K. (1995). Extraction of vegetation biophysical parameters by inversion of the PROSPECT + SAIL models on sugar beet canopy reflectance data. Application to TM and AVIRIS sensors. Remote Sensing of Environment, 52, 163-172

Jacquemoud, S., Verhoef, W., Baret, F., Bacour, C., Zarco-Tejada, P.J., Asner, G.P., François, C., \& Ustin, S.L. (2009). PROSPECT + SAIL models: A review of use for vegetation characterization. Remote Sensing of Environment, 113, Supplement $1, \mathrm{~S} 56-\mathrm{S} 66$

Jelalian, A.V. (1992). Laser radar systems. Artech House

Jennings, S., Brown, N., \& Sheil, D. (1999). Assessing forest canopies and understorey illumination: canopy closure, canopy cover and other measures. Forestry, 72, 5974

Jupp, D.L., Culvenor, D., Lovell, J.L., \& Newnham, G. (2005). Evaluation and validation of canopy laser radar (LIDAR) systems for native and plantation forest inventory. Final Report prepared for the Forest and Wood Products Research and Development Corporation (FWPRDC: PN 02.2902) by CSIRO, 20, 150

Jupp, D.L.B., Culvenor, D.S., Lovell, J.L., Newnham, G.J., Strahler, A.H., \& Woodcock, C.E. (2009). Estimating forest LAI profiles and structural parameters using a ground-based laser called 'Echidna ${ }^{\circledR}$. Tree Physiology, 29, 171-181

Jutzi, B., \& Gross, H. (2009). Normalization of LiDAR intensity data based on range and surface incidence angle. International Archives of Photogrammetry, Remote Sensing and Spatial Information Sciences, 38, 213-218 
Kaasalainen, S., Jaakkola, A., Kaasalainen, M., Krooks, A., \& Kukko, A. (2011). Analysis of incidence angle and distance effects on terrestrial laser scanner intensity: search for correction methods. Remote Sensing, 3, 2207-2221

Kaasalainen, S., Krooks, A., Kukko, A., \& Kaartinen, H. (2009). Radiometric calibration of terrestrial laser scanners with external reference targets. Remote Sensing, 1, 144-158

Kendall, M.G. (1946). The advanced theory of statistics. The advanced theory of statistics.

Khosravipour, A., Skidmore, A.K., \& Isenburg, M. (2016). Generating spike-free digital surface models using LiDAR raw point clouds: A new approach for forestry applications. International Journal of Applied Earth Observation and Geoinformation, 52, 104-114

Kim, S., McGaughey, R.J., Andersen, H.-E., \& Schreuder, G. (2009). Tree species differentiation using intensity data derived from leaf-on and leaf-off airborne laser scanner data. Remote Sensing of Environment, 113, 1575-1586

Kobayashi, H., \& Iwabuchi, H. (2008). A coupled 1-D atmosphere and 3-D canopy radiative transfer model for canopy reflectance, light environment, and photosynthesis simulation in a heterogeneous landscape. Remote Sensing of Environment, 112, 173-185

Koenig, K., Höfle, B., Hämmerle, M., Jarmer, T., Siegmann, B., \& Lilienthal, H. (2015). Comparative classification analysis of post-harvest growth detection from terrestrial LiDAR point clouds in precision agriculture. ISPRS Journal of Photogrammetry and Remote Sensing, 104, 112-125

Koetz, B., Baret, F., Poilvé, H., \& Hill, J. (2005). Use of coupled canopy structure dynamic and radiative transfer models to estimate biophysical canopy characteristics. Remote Sensing of Environment, 95, 115-124

Koetz, B., Morsdorf, F., van der Linden, S., Curt, T., \& Allgöwer, B. (2008). Multi-source land cover classification for forest fire management based on imaging spectrometry and LiDAR data. Forest Ecology and Management, 256, 263-271

Koetz, B., Sun, G., Morsdorf, F., Ranson, K.J., Kneubühler, M., Itten, K., \& Allgöwer, B. (2007). Fusion of imaging spectrometer and LIDAR data over combined radiative transfer models for forest canopy characterization. Remote Sensing of Environment, 106, 449-459

Korhonen, L., Korpela, I., Heiskanen, J., \& Maltamo, M. (2011). Airborne discrete-return LIDAR data in the estimation of vertical canopy cover, angular canopy closure and leaf area index. Remote Sensing of Environment, 115, 1065-1080

Kötz, B., Schaepman, M., Morsdorf, F., Bowyer, P., Itten, K., \& Allgöwer, B. (2004). Radiative transfer modeling within a heterogeneous canopy for estimation of forest fire fuel properties. Remote Sensing of Environment, 92, 332-344

Kramer, P.J. (1959). Transpiration and the water economy of plants. Plant physiology, 2, $607-726$

Krooks, A., Kaasalainen, S., Hakala, T., \& Nevalainen, O. (2013). Correction of intensity incidence angle effect in terrestrial laser scanning. ISPRS Annals of Photogrammetry, Remote Sensing and Spatial Information Sciences, 1, 145-150

Kukko, A., Kaasalainen, S., \& Litkey, P. (2008). Effect of incidence angle on laser scanner intensity and surface data. Applied Optics, 47, 986-992

Laurent, V.C.E., Verhoef, W., Clevers, J.G.P.W., \& Schaepman, M.E. (2011). Estimating forest variables from top-of-atmosphere radiance satellite measurements using 
coupled radiative transfer models. Remote Sensing of Environment, 115, 10431052

Lefsky, M.A., Cohen, W., Acker, S., Parker, G.G., Spies, T., \& Harding, D. (1999). Lidar remote sensing of the canopy structure and biophysical properties of Douglas-fir western hemlock forests. Remote Sensing of Environment, 70, 339-361

Li, X., \& Strahler, A.H. (1986). Geometric-Optical Bidirectional Reflectance Modeling of a Conifer Forest Canopy. Geoscience and Remote Sensing, IEEE Transactions on, $G E-24,906-919$

Li, X., Strahler, A.H., \& Woodcock, C.E. (1995). A hybrid geometric optical-radiative transfer approach for modeling albedo and directional reflectance of discontinuous canopies. IEEE Transactions on Geoscience and Remote Sensing, 33, 466-480

Li, Y., Guo, Q., Su, Y., Tao, S., Zhao, K., \& Xu, G. (2017). Retrieving the gap fraction, element clumping index, and leaf area index of individual trees using single-scan data from a terrestrial laser scanner. ISPRS Journal of Photogrammetry and Remote Sensing, 130, 308-316

Liang, S. (2005). Quantitative remote sensing of land surfaces. John Wiley \& Sons

Lidar, G.E.D.I. (2017). GEDI | Science Mission Directorate. In

Lim, K., Treitz, P., Wulder, M., St-Onge, B., \& Flood, M. (2003). LiDAR remote sensing of forest structure. Progress in physical Geography, 27, 88-106

Lin, Y.-C., \& Mills, J.P. (2010). Factors influencing pulse width of small footprint, full waveform airborne laser scanning data. Photogrammetric Engineering \& Remote Sensing, 76, 49-59

Lin, Y., \& Herold, M. (2016). Tree species classification based on explicit tree structure feature parameters derived from static terrestrial laser scanning data. Agricultural and Forest Meteorology, 216, 105-114

Lindberg, E., Olofsson, K., Holmgren, J., \& Olsson, H. (2012). Estimation of 3D vegetation structure from waveform and discrete return airborne laser scanning data. Remote Sensing of Environment, 118, 151-161

Liu, K., Shi, W., \& Zhang, H. (2011). A fuzzy topology-based maximum likelihood classification. ISPRS Journal of Photogrammetry and Remote Sensing, 66, 103114

Liu, S., Peng, Y., Du, W., Le, Y., \& Li, L. (2015). Remote estimation of leaf and canopy water content in winter wheat with different vertical distribution of water-related properties. Remote Sensing, 7, 4626-4650

Loudermilk, E.L., Hiers, J.K., O'Brien, J.J., Mitchell, R.J., Singhania, A., Fernandez, J.C., Cropper, W.P., \& Slatton, K.C. (2009). Ground-based LIDAR: a novel approach to quantify fine-scale fuelbed characteristics. International Journal of Wildland Fire, 18, 676-685

Loudermilk, E.L., Singhania, A., Fernandez, J.C., Hiers, J.K., O’Brien, J.J., Cropper Jr, W.P., Slatton, K.C., \& Mitchell, R.J. (2007). Application of ground-based LIDAR for fine-scale forest fuel modeling. USDA Forest Service Processing RMRS-P$46 C D$

Lovell, J., Jupp, D.L., Culvenor, D., \& Coops, N. (2003). Using airborne and groundbased ranging lidar to measure canopy structure in Australian forests. Canadian Journal of Remote Sensing, 29, 607-622

Lucas, R., Lee, A., \& Bunting, P. (2008). Retrieving forest biomass through integration of CASI and LiDAR data. International Journal of Remote Sensing, 29, 15531577 
Ma, H., Song, J., Wang, J., Xiao, Z., \& Fu, Z. (2014). Improvement of spatially continuous forest LAI retrieval by integration of discrete airborne LiDAR and remote sensing multi-angle optical data. Agricultural and Forest Meteorology, 189-190, 60-70

Ma, L., Zheng, G., Eitel, J.U.H., Magney, T.S., \& Moskal, L.M. (2016a). Determining woody-to-total area ratio using terrestrial laser scanning (TLS). Agricultural and Forest Meteorology, 228-229, 217-228

Ma, L., Zheng, G., Eitel, J.U.H., Moskal, L.M., He, W., \& Huang, H. (2016b). Improved salient feature-based approach for automatically separating photosynthetic and nonphotosynthetic components within terrestrial lidar point cloud data of forest canopies. IEEE Transactions on Geoscience and Remote Sensing, 54, 679-696

Magney, T.S., Eusden, S.A., Eitel, J.U.H., Logan, B.A., Jiang, J., \& Vierling, L.A. (2014). Assessing leaf photoprotective mechanisms using terrestrial LiDAR: towards mapping canopy photosynthetic performance in three dimensions. New Phytologist, 201, 344-356

Mallet, C., \& Bretar, F. (2009). Full-waveform topographic lidar: State-of-the-art. ISPRS Journal of Photogrammetry and Remote Sensing, 64, 1-16

Mallet, C., Bretar, F., Roux, M., Soergel, U., \& Heipke, C. (2011). Relevance assessment of full-waveform lidar data for urban area classification. ISPRS Journal of Photogrammetry and Remote Sensing, 66, S71-S84

Martinuzzi, S., Vierling, L.A., Gould, W.A., Falkowski, M.J., Evans, J.S., Hudak, A.T., \& Vierling, K.T. (2009). Mapping snags and understory shrubs for a LiDAR-based assessment of wildlife habitat suitability. Remote Sensing of Environment, 113, 2533-2546

Meroni, M., Colombo, R., \& Panigada, C. (2004). Inversion of a radiative transfer model with hyperspectral observations for LAI mapping in poplar plantations. Remote Sensing of Environment, 92, 195-206

Mirzaie, M., Darvishzadeh, R., Shakiba, A., Matkan, A., Atzberger, C., \& Skidmore, A. (2014). Comparative analysis of different uni-and multi-variate methods for estimation of vegetation water content using hyper-spectral measurements. International Journal of Applied Earth Observation and Geoinformation, 26, 111

Moeser, D., Roubinek, J., Schleppi, P., Morsdorf, F., \& Jonas, T. (2014). Canopy closure, LAI and radiation transfer from airborne LiDAR synthetic images. Agricultural and Forest Meteorology, 197, 158-168

Mooney, H., Ehleringer, J., \& Björkman, O. (1977). The energy balance of leaves of the evergreen desert shrub Atriplex hymenelytra. Oecologia, 29, 301-310

Moorthy, I., Miller, J.R., Hu, B., Chen, J., \& Li, Q. (2008). Retrieving crown leaf area index from an individual tree using ground-based lidar data. Canadian Journal of Remote Sensing, 34, 320-332

Morsdorf, F., Kötz, B., Meier, E., Itten, K.I., \& Allgöwer, B. (2006). Estimation of LAI and fractional cover from small footprint airborne laser scanning data based on gap fraction. Remote Sensing of Environment, 104, 50-61

Morsdorf, F., Meier, E., Kötz, B., Itten, K.I., Dobbertin, M., \& Allgöwer, B. (2004). LIDAR-based geometric reconstruction of boreal type forest stands at single tree level for forest and wildland fire management. Remote Sensing of Environment, 92, 353-362 
Mutanga, O., \& Skidmore, A.K. (2004). Integrating imaging spectroscopy and neural networks to map grass quality in the Kruger National Park, South Africa. Remote Sensing of Environment, 90, 104-115

Ni, H., Lin, X., \& Zhang, J. (2017). Classification of ALS Point Cloud with Improved Point Cloud Segmentation and Random Forests. Remote Sensing, 9, 288

Niemann, K.O., Quinn, G., Goodenough, D.G., Visintini, F., \& Loos, R. (2012). Addressing the effects of canopy structure on the remote sensing of foliar chemistry of a 3-dimensional, radiometrically porous surface. IEEE Journal of Selected Topics in Applied Earth Observations and Remote Sensing, 5, 584-593

Nobis, M., \& Hunziker, U. (2005). Automatic thresholding for hemispherical canopyphotographs based on edge detection. Agricultural and Forest Meteorology, 128, 243-250

North, P. (1996). Three-dimensional forest light interaction model using a Monte Carlo method. Geoscience and Remote Sensing, IEEE Transactions on, 34, 946-956

North, P.R.J., Rosette, J.A.B., Suárez, J.C., \& Los, S.O. (2010). A Monte Carlo radiative transfer model of satellite waveform LiDAR. International Journal of Remote Sensing, 31, 1343-1358

Ollinger, S.V. (2011). Sources of variability in canopy reflectance and the convergent properties of plants. New Phytologist, 189, 375-394

Ørka, H.O., Næsset, E., \& Bollandsås, O.M. (2009). Classifying species of individual trees by intensity and structure features derived from airborne laser scanner data. Remote Sensing of Environment, 113, 1163-1174

Pal, M. (2005). Random forest classifier for remote sensing classification. International Journal of Remote Sensing, 26, 217-222

Pearson, K. (1896). Mathematical contributions to the theory of evolution. III. Regression, heredity, and panmixia. Philosophical Transactions of the Royal Society of London. Series A, Containing Papers of a Mathematical or Physical Character, 187, 253-318

Pedregosa, F., Varoquaux, G., Gramfort, A., Michel, V., Thirion, B., Grisel, O., Blondel, M., Prettenhofer, P., Weiss, R., \& Dubourg, V. (2011). Scikit-learn: Machine learning in Python. Journal of Machine Learning Research, 12, 2825-2830

Penasa, L., Franceschi, M., Preto, N., Teza, G., \& Polito, V. (2014). Integration of intensity textures and local geometry descriptors from Terrestrial Laser Scanning to map chert in outcrops. ISPRS Journal of Photogrammetry and Remote Sensing, 93, 88-97

Peñuelas, J., Filella, I., Biel, C., Serrano, L., \& Save, R. (1993). The reflectance at the 950-970 $\mathrm{nm}$ region as an indicator of plant water status. International Journal of Remote Sensing, 14, 1887-1905

Pesci, A., \& Teza, G. (2008). Effects of surface irregularities on intensity data from laser scanning: an experimental approach. Annals of Geophysics, 51, 839-848

Pfennigbauer, M., \& Ullrich, A. (2010). Improving quality of laser scanning data acquisition through calibrated amplitude and pulse deviation measurement. In, Proc. SPIE 7684, Laser Radar Technology and Applications XV (p. 76841F)

Pfennigbauer, M., Wolf, C., \& Ullrich, A. (2013). Enhancing online waveform processing by adding new point attributes. In, Proc. SPIE (pp. 873104-873101-873109)

Pirotti, F., Guarnieri, A., \& Vettore, A. (2013). Ground filtering and vegetation mapping using multi-return terrestrial laser scanning. ISPRS Journal of Photogrammetry and Remote Sensing, 76, 56-63 
Poullain, E., Garestier, F., Bretel, P., \& Levoy, F. (2012). Modeling of als intensity behavior as a function of incidence angle for coastal zone surface study. In, Geoscience and Remote Sensing Symposium (IGARSS), 2012 IEEE International (pp. 2849-2852)

Rabas, A.R., \& Martin, C.E. (2003). Movement of Water from Old to Young Leaves in Three Species of Succulents. Annals of Botany, 92, 529-536

Rautiainen, M. (2005). Retrieval of leaf area index for a coniferous forest by inverting a forest reflectance model. Remote Sensing of Environment, 99, 295-303

Ray, D., Nepstad, D., \& Moutinho, P. (2005). Micrometeorological and canopy controls of fire susceptibility in a forested Amazon landscape. Ecological Applications, 15, $1664-1678$

Reitberger, J., Krzystek, P., \& Stilla, U. (2008). Analysis of full waveform LIDAR data for the classification of deciduous and coniferous trees. International Journal of Remote Sensing, 29, 1407-1431

Reitberger, J., Schnörr, C., Krzystek, P., \& Stilla, U. (2009). 3D segmentation of single trees exploiting full waveform LIDAR data. ISPRS Journal of Photogrammetry and Remote Sensing, 64, 561-574

Richter, K., Vuolo, F., D'Urso, G., \& Dini, L. (2007). Evaluation of different methods for the retrieval of LAI using high resolution airborne data. In, Proceedings of SPIE, the International Society for Optical Engineering (pp. 67420E. 67421-67420E. 67410): Society of Photo-Optical Instrumentation Engineers

Rosema, A., Verhoef, W., Noorbergen, H., \& Borgesius, J.J. (1992). A new forest light interaction model in support of forest monitoring. Remote Sensing of Environment, $42,23-41$

Roth-Nebelsick, A., Uhl, D., Mosbrugger, V., \& Kerp, H. (2001). Evolution and function of leaf venation architecture: a review. Annals of Botany, 87, 553-566

Rothermel, R.C., Wilson, R., Morris, G.A., \& Sackett, S.S. (1986). Modeling moisture content of fine dead wildland fuels. USDA Forest Service research paper. INT, 359

Running, S.W., \& Gower, S.T. (1991). FOREST-BGC, a general model of forest ecosystem processes for regional applications. II. Dynamic carbon allocation and nitrogen budgets. Tree Physiology, 9, 147-160

Saatchi, S., Halligan, K., Despain, D.G., \& Crabtree, R.L. (2007). Estimation of forest fuel load from radar remote sensing. IEEE Transactions on Geoscience and Remote Sensing, 45, 1726-1740

Sanchez, R.A., Hall, A.J., Trapani, N., \& de Hunau, R.C. (1983). Effects of water stress on the chlorophyll content, nitrogen level and photosynthesis of leaves of two maize genotypes. Photosynthesis Research, 4, 35-47

Schlerf, M., \& Atzberger, C. (2006). Inversion of a forest reflectance model to estimate structural canopy variables from hyperspectral remote sensing data. Remote Sensing of Environment, 100, 281-294

Schlerf, M., \& Atzberger, C. (2012). Vegetation structure retrieval in beech and spruce forests using spectrodirectional satellite data. IEEE Journal of Selected Topics in Applied Earth Observations and Remote Sensing, 5, 8-17

Scriber, J.M. (1977a). Limiting effects of low leaf-water content on the nitrogen utilization, energy budget, and larval growth of Hyalophora cecropia (Lepidoptera: Saturniidae). Oecologia, 28, 269-287 
Scriber, J.M. (1977b). Limiting effects of low leaf-water content on the nitrogen utilization, energy budget, and larval growth ofHyalophora cecropia (Lepidoptera: Saturniidae). Oecologia, 28, 269-287

Seelig, H.D., Hoehn, A., Stodieck, L.S., Klaus, D.M., Adams Iii, W.W., \& Emery, W.J. (2008). Relations of remote sensing leaf water indices to leaf water thickness in cowpea, bean, and sugarbeet plants. Remote Sensing of Environment, 112, 445455

Serrano, L., Ustin, S.L., Roberts, D.A., Gamon, J.A., \& Penuelas, J. (2000). Deriving water content of chaparral vegetation from AVIRIS data. Remote Sensing of Environment, 74, 570-581

Sinclair, T., Schreiber, M., \& Hoffer, R. (1973). Diffuse reflectance hypothesis for the pathway of solar radiation through leaves. Agronomy Journal, 65, 276-283

Skidmore, A., Pettorelli, N., Coops, N., Geller, G., Hansen, M., Lucas, R., Mücher, C., O'Connor, B., Paganini, M., \& Pereira, H. (2015). Environmental science: Agree on biodiversity metrics to track from space. Nature, 523, 403

Skidmore, A.K., Ferwerda, J.G., Mutanga, O., Van Wieren, S.E., Peel, M., Grant, R.C., Prins, H.H., Balcik, F.B., \& Venus, V. (2010). Forage quality of savannasSimultaneously mapping foliar protein and polyphenols for trees and grass using hyperspectral imagery. Remote Sensing of Environment, 114, 64-72

Solberg, S., Brunner, A., Hanssen, K.H., Lange, H., Næsset, E., Rautiainen, M., \& Stenberg, P. (2009). Mapping LAI in a Norway spruce forest using airborne laser scanning. Remote Sensing of Environment, 113, 2317-2327

Solberg, S., Hill, R., Rosette, J., \& Suárez, J. (2008). Comparing discrete echoes counts and intensity sums from ALS for estimating forest LAI and gap fraction. In, Proceedings of SilviLaser 2008, 8th international conference on LiDAR applications in forest assessment and inventory, Heriot-Watt University, Edinburgh, UK, 17-19 September, 2008. (pp. 247-256): SilviLaser 2008 Organizing Committee

Spies, T.A. (1998). Forest structure: a key to the ecosystem. Northwest science, 72, 3436

St-Onge, B., \& Budei, B. (2015). Individual tree species identification using the multispectral return intensities of the Optech Titan LiDAR system. Proceedings of SilviLaser, 28-30

Stancik, A.L., \& Brauns, E.B. (2008). A simple asymmetric lineshape for fitting infrared absorption spectra. Vibrational Spectroscopy, 47, 66-69

Strahler, A.H., Jupp, D.L., Woodcock, C.E., Schaaf, C.B., Yao, T., Zhao, F., Yang, X., Lovell, J., Culvenor, D., \& Newnham, G. (2008). Retrieval of forest structural parameters using a ground-based lidar instrument (Echidna $\left.{ }^{\circledR}\right)$. Canadian Journal of Remote Sensing, 34, S426-S440

Suits, G.H. (1971). The calculation of the directional reflectance of a vegetative canopy. Remote Sensing of Environment, 2, 117-125

Tang, H., Brolly, M., Zhao, F., Strahler, A.H., Schaaf, C.L., Ganguly, S., Zhang, G., \& Dubayah, R. (2014a). Deriving and validating Leaf Area Index (LAI) at multiple spatial scales through lidar remote sensing: A case study in Sierra National Forest, CA. Remote Sensing of Environment, 143, 131-141

Tang, H., Brolly, M., Zhao, F., Strahler, A.H., Schaaf, C.L., Ganguly, S., Zhang, G., \& Dubayah, R. (2014b). Deriving and validating Leaf Area Index (LAI) at multiple 
spatial scales through lidar remote sensing: A case study in Sierra National Forest, CA. Remote Sensing of Environment, 143, 131-141

Tang, H., Dubayah, R., Swatantran, A., Hofton, M., Sheldon, S., Clark, D.B., \& Blair, B. (2012). Retrieval of vertical LAI profiles over tropical rain forests using waveform lidar at La Selva, Costa Rica. Remote Sensing of Environment, 124, 242-250

Thimonier, A., Sedivy, I., \& Schleppi, P. (2010). Estimating leaf area index in different types of mature forest stands in Switzerland: a comparison of methods. European Journal of Forest Research, 129, 543-562

Thomas, V., Treitz, P., McCaughey, J.H., Noland, T., \& Rich, L. (2007). Canopy chlorophyll concentration estimation using hyperspectral and lidar data for a boreal mixedwood forest in northern Ontario, Canada. International Journal of Remote Sensing, 29, 1029-1052

Tong, A., \& He, Y. (2017). Estimating and mapping chlorophyll content for a heterogeneous grassland: Comparing prediction power of a suite of vegetation indices across scales between years. ISPRS Journal of Photogrammetry and Remote Sensing, 126, 146-167

Torabzadeh, H., Morsdorf, F., Leiterer, R., \& Schaepman, M.E. (2014). Fusing imaging spectrometry and airborne laser scanning data for tree species discrimination. In, Geoscience and Remote Sensing Symposium (IGARSS), 2014 IEEE International (pp. 1253-1256): IEEE

Treitz, P.M., \& Howarth, P.J. (1999). Hyperspectral remote sensing for estimating biophysical parameters of forest ecosystems. Progress in physical Geography, 23, 359-390

Tsunoda, T., Kachi, N., \& Suzuki, J.I. (2014). Availability and temporal heterogeneity of water supply affect the vertical distribution and mortality of a belowground herbivore and consequently plant growth. Plos One, 9

Tucker, C.J. (1980). Remote sensing of leaf water content in the near infrared. Remote Sensing of Environment, 10, 23-32

Ullah, S., Skidmore, A.K., Groen, T.A., \& Schlerf, M. (2013). Evaluation of three proposed indices for the retrieval of leaf water content from the mid-wave infrared (2-6 $\mu \mathrm{m})$ spectra. Agricultural and Forest Meteorology, 171-172, 65-71

Ullah, S., Skidmore, A.K., Naeem, M., \& Schlerf, M. (2012). An accurate retrieval of leaf water content from mid to thermal infrared spectra using continuous wavelet analysis. Science of The Total Environment, 437, 145-152

Ullah, S., Skidmore, A.K., Ramoelo, A., Groen, T.A., Naeem, M., \& Ali, A. (2014). Retrieval of leaf water content spanning the visible to thermal infrared spectra. ISPRS Journal of Photogrammetry and Remote Sensing, 93, 56-64

Us Department of Commerce, N.O., \& Atmospheric, A. (2012). What is LIDAR. In

Ustin, S.L., Roberts, D.A., Pinzon, J., Jacquemoud, S., Gardner, M., Scheer, G., Castaneda, C.M., \& Palacios-Orueta, A. (1998). Estimating canopy water content of chaparral shrubs using optical methods. Remote Sensing of Environment, 65, 280-291

Verhoef, W. (1984). Light scattering by leaf layers with application to canopy reflectance modeling: The SAIL model. Remote Sensing of Environment, 16, 125-141

Verhoef, W. (1985). Earth observation modeling based on layer scattering matrices. Remote Sensing of Environment, 17, 165-178 
Verhoef, W., \& Bach, H. (2003). Simulation of hyperspectral and directional radiance images using coupled biophysical and atmospheric radiative transfer models. Remote Sensing of Environment, 87, 23-41

Verhoef, W., \& Bach, H. (2007). Coupled soil-leaf-canopy and atmosphere radiative transfer modeling to simulate hyperspectral multi-angular surface reflectance and TOA radiance data. Remote Sensing of Environment, 109, 166-182

Vierling, L.A., Xu, Y., Eitel, J.U., \& Oldow, J.S. (2013). Shrub characterization using terrestrial laser scanning and implications for airborne LiDAR assessment. Canadian Journal of Remote Sensing, 38, 709-722

Vosselman, G., \& Maas, H.-G. (2010). Airborne and terrestrial laser scanning. Whittles Publishing

Wagner, W. (2010). Radiometric calibration of small-footprint full-waveform airborne laser scanner measurements: Basic physical concepts. ISPRS Journal of Photogrammetry and Remote Sensing, 65, 505-513

Wagner, W., Hollaus, M., Briese, C., \& Ducic, V. (2008a). 3D vegetation mapping using small-footprint full-waveform airborne laser scanners. International Journal of Remote Sensing, 29, 1433-1452

Wagner, W., Hyyppä, J., Ullrich, A., Lehner, H., Briese, C., \& Kaasalainen, S. (2008b). Radiometric calibration of full-waveform small-footprint airborne laser scanners. International Archives of Photogrammetry, Remote Sensing and Spatial Information Sciences, 37, 163-168

Wagner, W., Ullrich, A., Ducic, V., Melzer, T., \& Studnicka, N. (2006). Gaussian decomposition and calibration of a novel small-footprint full-waveform digitising airborne laser scanner. ISPRS Journal of Photogrammetry and Remote Sensing, $60,100-112$

Wallace, A., Nichol, C., \& Woodhouse, I. (2012). Recovery of Forest Canopy Parameters by Inversion of Multispectral LiDAR Data. Remote Sensing, 4, 509

Wang, Q., \& Li, P. (2013). Canopy vertical heterogeneity plays a critical role in reflectance simulation. Agricultural and Forest Meteorology, 169, 111-121

Wang, Z., Skidmore, A.K., Wang, T., Darvishzadeh, R., Heiden, U., Heurich, M., Latifi, H., \& Hearne, J. (2017). Canopy foliar nitrogen retrieved from airborne hyperspectral imagery by correcting for canopy structure effects. International Journal of Applied Earth Observation and Geoinformation, 54, 84-94

Wang, Z., Wang, T., Darvishzadeh, R., Skidmore, A.K., Jones, S., Suarez, L., Woodgate, W., Heiden, U., Heurich, M., \& Hearne, J. (2016). Vegetation indices for mapping canopy foliar nitrogen in a mixed temperate forest. Remote Sensing, 8, 491

Wessman, C. (1994). Estimating canopy biochemistry through imaging spectrometry. In J. Hill, \& J. Mégier (Eds.), Imaging Spectrometry - A Tool For Environmental Observations (pp. 57-69). Netherlands: Springer Netherlands

Widlowski, J., Verstraete, M., Pinty, B., \& Gobron, N. (2003). Allometric relationships of selected european tree species: Parametrizations of tree architecture for the purpose of 3-d canopy reflectance models used in the interpretation of remote sensing: Betula pubescens. Fagus sylvatica, Larix decidua, Picea abies, Pinus sylvestris

Woodhouse, I.H., Nichol, C., Sinclair, P., Jack, J., Morsdorf, F., Malthus, T.J., \& Patenaude, G. (2011). A multispectral canopy LiDAR demonstrator project. IEEE Geoscience and Remote Sensing Letters, 8, 839-843 
Xiao, Y., Zhao, W., Zhou, D., \& Gong, H. (2014). Sensitivity analysis of vegetation reflectance to biochemical and biophysical variables at leaf, canopy, and regional scales. IEEE Transactions on Geoscience and Remote Sensing, 52, 4014-4024

Yamasaki, S., \& Dillenburg, L.R. (1999). Measurements of leaf relative water content in Araucaria angustifolia. Revista Brasilleira de Fisiologia Vegetal, 11, 69-75

Yang, B., \& Dong, Z. (2013). A shape-based segmentation method for mobile laser scanning point clouds. ISPRS Journal of Photogrammetry and Remote Sensing, $81,19-30$

Yang, G., Zhao, C., Liu, Q., Huang, W., \& Wang, J. (2011). Inversion of a radiative transfer model for estimating forest lai from multisource and multiangular optical remote sensing data. IEEE Transactions on Geoscience and Remote Sensing, 49, 988-1000

Yao, W., Krzystek, P., \& Heurich, M. (2012). Tree species classification and estimation of stem volume and DBH based on single tree extraction by exploiting airborne full-waveform LiDAR data. Remote Sensing of Environment, 123, 368-380

Yebra, M., \& Chuvieco, E. (2009). Linking ecological information and radiative transfer models to estimate fuel moisture content in the Mediterranean region of Spain: Solving the ill-posed inverse problem. Remote Sensing of Environment, 113, 24032411

Yi, Q.-x., Bao, A.-m., Wang, Q., \& Zhao, J. (2013). Estimation of leaf water content in cotton by means of hyperspectral indices. Computers and Electronics in Agriculture, 90, 144-151

Yi, Q., Wang, F., Bao, A., \& Jiapaer, G. (2014). Leaf and canopy water content estimation in cotton using hyperspectral indices and radiative transfer models. International Journal of Applied Earth Observation and Geoinformation, 33, 67-75

Yu, X., Hyyppä, J., Litkey, P., Kaartinen, H., Vastaranta, M., \& Holopainen, M. (2017). Single-Sensor Solution to Tree Species Classification Using Multispectral Airborne Laser Scanning. Remote Sensing, 9, 108

Zarco-Tejada, P.J., Miller, J.R., Morales, A., Berjón, A., \& Agüera, J. (2004). Hyperspectral indices and model simulation for chlorophyll estimation in opencanopy tree crops. Remote Sensing of Environment, 90, 463-476

Zarco-Tejada, P.J., Rueda, C.A., \& Ustin, S.L. (2003). Water content estimation in vegetation with MODIS reflectance data and model inversion methods. Remote Sensing of Environment, 85, 109-124

Zhang, L., Zhou, Z., Zhang, G., Meng, Y., Chen, B., \& Wang, Y. (2012). Monitoring the leaf water content and specific leaf weight of cotton (Gossypium hirsutum L.) in saline soil using leaf spectral reflectance. European Journal of Agronomy, 41, 103117

Zhang, Y., Zhu, H., Yan, B., Ou, Y., \& Li, X. (2013). Vertical distribution and temporal variation of nitrogen, phosphorus and carbon in ditch sediment of Sanjiang plain, northeast China. Fresenius Environmental Bulletin, 22, 2265-2272

Zhang, Z., \& Liu, X. (2012). Support vector machines for tree species identification using LiDAR-derived structure and intensity variables. Geocarto International, 28, 364378

Zhao, F., Yang, X., Schull, M.A., Román-Colón, M.O., Yao, T., Wang, Z., Zhang, Q., Jupp, D.L.B., Lovell, J.L., Culvenor, D.S., Newnham, G.J., Richardson, A.D., NiMeister, W., Schaaf, C.L., Woodcock, C.E., \& Strahler, A.H. (2011). Measuring effective leaf area index, foliage profile, and stand height in New England forest 
stands using a full-waveform ground-based lidar. Remote Sensing of Environment, 115, 2954-2964

Zhao, K., Popescu, S., \& Nelson, R. (2009). Lidar remote sensing of forest biomass: A scale-invariant estimation approach using airborne lasers. Remote Sensing of Environment, 113, 182-196

Zheng, G., Ma, L., He, W., Eitel, J.U., Moskal, L.M., \& Zhang, Z. (2016a). Assessing the contribution of woody materials to forest angular gap fraction and effective leaf area index using terrestrial laser scanning data. IEEE Transactions on Geoscience and Remote Sensing, 54, 1475-1487

Zheng, G., Ma, L.X., He, W., Eitel, J.U.H., Moskal, L.M., \& Zhang, Z.Y. (2016b). Assessing the Contribution of Woody Materials to Forest Angular Gap Fraction and Effective Leaf Area Index Using Terrestrial Laser Scanning Data. IEEE Transactions on Geoscience and Remote Sensing, 54, 1475-1487

Zhu, X., Skidmore, A.K., Darvishzadeh, R., Niemann, K.O., Liu, J., Shi, Y., \& Wang, T. (2018). Foliar and woody materials discriminated using terrestrial LiDAR in a mixed natural forest. International Journal of Applied Earth Observation and Geoinformation, 64, 43-50

Zhu, X., Wang, T., Darvishzadeh, R., Skidmore, A.K., \& Niemann, K.O. (2015). 3D leaf water content mapping using terrestrial laser scanner backscatter intensity with radiometric correction. ISPRS Journal of Photogrammetry and Remote Sensing, $110,14-23$

Zhu, X., Wang, T., Skidmore, A.K., Darvishzadeh, R., Niemann, K.O., \& Liu, J. (2017). Canopy leaf water content estimated using terrestrial LiDAR. Agricultural and Forest Meteorology, 232, 152-162 


\section{Summary}

Forest leaf water content (LWC) plays a key role in many physiological processes such as plant growth, photosynthesis, transpiration, and thermal regulation. The estimation of LWC and knowledge about its spatial variation can provide important information for assessing forest drought conditions and predicting future forest change associated with climate change. Recently it was proposed as one of the essential biodiversity variables (EBVs) to assess progress towards the Aichi Biodiversity Targets.

Remote sensing data have been proved effective to provide a non-destructive, rapid and economical way for the estimation of LWC at various scales. At the leaf scale, terrestrial laser scanning (TLS) data enable vegetation information to be explored at small spatial scales $(\mathrm{mm})$. The backscatter intensity value of the reflected backscatter signal recorded by TLS is, to some extent, insensitive to ambient light and atmospheric conditions and provides good spectral separability for detecting and classifying objects. At the individual canopy scale, TLS data are capable of providing vertical information of LWC within canopies. However, at the regional scale, unlike TLS, due to the large footprint size of airborne laser scanning (ALS), most laser beams hit multiple targets within the footprint. With insufficient prior knowledge about full hits from the same target, the true reflectance of partially hit individual targets cannot be unmixed because the target reflectance and the collision area between the laser beam and partial hits both contribute to the returned intensity.

The thesis aimed to estimate LWC at the leaf, canopy and regional scales using LiDAR and/or hyperspectral data. The research in the thesis firstly presented a new method to eliminate the incidence angle effects on TLS intensity data. The results showed that LWC and its 3D spatial distribution can be estimated at a moderate accuracy using corrected TLS intensity data. Secondly, the thesis evaluated the partial hits and incidence angle effects on LWC estimation at the individual canopy scale. LWC vertical distribution within plant canopy can be retrieved from TLS. To quantify the impact of woody material on the estimation of LWC, the thesis subsequently investigated a new algorithm to classify foliar and woody materials using TLS data. An adaptive radius near-neighbor search algorithm was employed to extract both geometric and radiometric features. The findings suggested that an accurate classification between foliar and woody materials can be achieved in a mixed natural forest by using TLS. Then the research moved on to the regional scale with the emphasis on removing canopy structural and background effects on LWC estimation from airborne LiDAR and hyperspectral data using radiative transfer models. Prior knowledge about canopy cover and background reflectance using the integration of LiDAR and hyperspectral data was used in the inversion of radiative transfer models. The results showed that the structural and background effects could be effectively minimized and the accuracy of LWC estimation was significantly improved. 
This thesis explored the potential of LiDAR remote sensing alone for LWC estimation at the leaf and individual canopy scales, and the combination of LiDAR and hyperspectral data for LWC estimation at the regional scale. It demonstrates that the LWC distribution within individual leaves and the vertical distribution of LWC within vegetation canopy can be estimated using terrestrial LiDAR, and the prior information derived from LiDAR and hyperspectral data can significantly improve the estimation of LWC through the inversion of radiative transfer models at the regional scale. Using the calibration methods developed in this thesis, the approaches have the potential to be transferred to other sites. Global maps of LWC can be generated when satellites data such as the Global Ecosystem Dynamics Investigation (GEDI) LiDAR become operational. 


\section{Samenvatting}

Blad-watergehalte speelt een sleutelrol in veel fysiologische processen, zoals plantengroei, fotosynthese, transpiratie en thermische regulatie. De inschatting van bladwatergehalte en kennis over de ruimtelijke variatie ervan kan belangrijke informatie opleveren voor het beoordelen van bos-droogtecondities en het voorspellen van toekomstige verandering van het bos in verband met klimaatverandering. Onlangs werd bovenstaande voorgesteld als een van de essentiële biodiversiteitsvariabelen om de voortgang van de Aichi-biodiversiteitsdoelen te beoordelen.

Gegevens van teledetectie zijn effectief gebleken om een niet-destructieve, snelle en economische manier te bieden voor de schatting van blad-watergehalte op verschillende schalen. Op het blad-niveau maken terrestrische laserscanning (TLS) gegevens het verkennen van kleine ruimtelijke schaal mogelijk. De intensiteitswaarde van het gereflecteerde terugverstrooiing signaal geregistreerd door TLS is in zekere mate ongevoelig voor omgevingslicht en atmosferische omstandigheden, en biedt een goede spectrale scheidbaarheid voor het detecteren en classificeren van objecten. Op de individuele canopy-schaal kunnen TLS-gegevens verticale informatie van LWC binnen blad-luifels leveren. Op regionale schaal, in tegenstelling tot TLS, hebben de meeste laserstralen echter door de grote gebieds-grootte van laserstralen in de lucht worden verschillende doelen binnen het gebied geraakt. Met onvoldoende voorkennis over volledige treffers van hetzelfde doel, kan de werkelijke reflectie van gedeeltelijk getroffen individuele doelen niet uniform zijn, aangezien de reflectie van het doel en het botsingsgebied tussen de laserstraal en gedeeltelijke treffers beide aan de gereflecteerde intensiteit bijdragen.

Het proefschrift was gericht op het schatten van blad watergehalte op de blad-, kruin- en regionale schalen met behulp van LiDAR en/of hyperspectrale gegevens. Ten eerst presenteerde het onderzoek in het proefschrift een nieuwe methode om de incidentiehoekeffecten op TLS-intensiteitsgegevens te elimineren. De resultaten toonden aan dat blad-watergehalte en zijn ruimtelijke 3D-verdeling op gematigde nauwkeurigheid met behulp van gecorrigeerde TLS-intensiteitsgegevens kunnen worden geschat.

Ten tweede evalueerde het proefschrift de partiële treffers en incidentiehoekeffecten op de schatting van blad watergehalte op de individuele blad-luifelschaal. Verticale verdeling van blad-watergehalte binnen het bladerdak van de plant kan door TLS worden verkregen. Om de impact van houtachtig materiaal te kunnen kwantificeren op basis van de schatting van LWC, onderzocht het proefschrift vervolgens een nieuw algoritme om blad- en houtachtige materialen te classificeren met behulp van TLS-gegevens. Een adaptief radius near-neighbour search-algoritme werd gebruikt om zowel geometrische als radiometrische kenmerken te extraheren. De bevindingen suggereerden dat een 
nauwkeurige classificatie tussen blad- en houtachtige materialen in een gemengd natuurlijk bos met behulp van TLS kan worden bereikt.

Ten derde werd het onderzoek voortgezet op regionale schaal met de nadruk op het verwijderen van luifel structurele en achtergrondeffecten op basis van de LWC schatting en van in de lucht aanwezige LiDAR en hyperspectrale gegevens met behulp van stralingsoverdrachtmodellen. Voorafgaande kennis over luifeldekking en achtergrondreflectie met behulp van de integratie van LiDAR en hyperspectrale gegevens werd gebruikt bij de omkering van radiatieve overdrachtsmodellen. De resultaten toonden aan dat de structurele en achtergrondeffecten effectief konden worden geminimaliseerd en de nauwkeurigheid van de schatting van blad watergehalte aanzienlijk werd verbeterd.

Dit proefschrift onderzocht het potentieel van alleen LiDAR remote sensing voor de schatting van blad watergehalte op de blad- en individuele luifels, alsmede de combinatie van LiDAR en hyperspectrale gegevens voor de schatting van blad watergehalte op regionale schaal. Het toont aan dat de verdeling van de blad watergehalte binnen individuele bladeren en de verticale verdeling van blad watergehalte binnen de begroeiing van vegetatie met behulp van terrestrische LiDAR kan worden geschat, en dat de voorafgaande informatie afgeleid van LiDAR en hyperspectrale gegevens de schatting van LWC aanzienlijk door de omvorming van modellen voor stralingsoverdracht op regionale schaal kan verbeteren. Met behulp van de kalibratiemethoden die in dit proefschrift zijn ontwikkeld, kunnen de benaderingen naar andere sites worden overgedragen. Globale kaarten van blad watergehalte kunnen worden gegenereerd wanneer satellietsgegevens zoals de Global Ecosystem Dynamics Investigation (GEDI) LiDAR operationeel worden. 


\section{Biography}

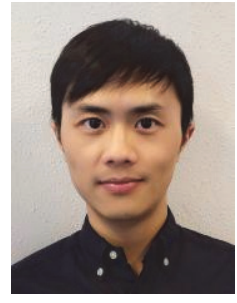

Xi Zhu was born on 18 June 1987 in Anhui, China. He received his BSc degree in Forestry in Beijing Forestry University, and his MSc degree in Forest Remote Sensing from Research Institute of Forest Resource Information Techniques, Chinese Academy of Forestry. In 2013, he started to pursue his PhD degree at the Faculty of GeoInformation Science and Earth Observation (ITC), University of Twente. His research is focused on the exploration of LiDAR and hyperspectral data in forest applications.

\section{Peer-reviewed journal papers:}

Zhu, X., Wang, T., Darvishzadeh, R., Skidmore, A.K., \& Niemann, K.O. (2015). 3D leaf water content mapping using terrestrial laser scanner backscatter intensity with radiometric correction. ISPRS Journal of Photogrammetry and Remote Sensing, $110,14-23$.

Zhu, X., Wang, T., Skidmore, A.K., Darvishzadeh, R., Niemann, K.O., \& Liu, J. (2017). Canopy leaf water content estimated using terrestrial LiDAR. Agricultural and Forest Meteorology, 232, 152-162.

Zhu, X., Skidmore, A.K., Darvishzadeh, R., Niemann, K.O., Liu, J., Shi, Y., \& Wang, T. (2018). Foliar and woody materials discriminated using terrestrial LiDAR in a mixed natural forest. International Journal of Applied Earth Observation and Geoinformation, 64, 43-50.

Zhu, X., Skidmore, A.K., Darvishzadeh, R., \& Wang, T. (under review). Retrieval of leaf water content from airborne hyperspectral and LiDAR data. ISPRS Journal of Photogrammetry and Remote Sensing.

Zhu, X., Skidmore, A.K., Wang, T., Liu, J., Darvishzadeh, R., Shi Y., Premier J., \& Heurich, M. (under review). Improving leaf area index (LAI) estimation by correcting for foliage clumping and woody effects using terrestrial laser scanning. Agricultural and Forest Meteorology.

Liu, J., Skidmore, A.K., Jones, S., Wang, T., Heurich, M., Zhu, X., Shi, Y. (2018). Large off-nadir scan angle of airborne LiDAR can severely affect the estimates of forest structure metrics. ISPRS Journal of Photogrammetry and Remote Sensing, $136,13-25$.

\section{Conference Proceedings:}

Zhu, X., Skidmore, A. K., Darvishzadeh, R., \& Wang, T. (2015). Detecting leaf water content using intensity data from terrestrial laser scanner. In SilviLaser 2015, 28-30 September 2015, La Grande Motte, France.

Zhu, X., Skidmore, A. K., Darvishzadeh, R., \& Wang, T. (2016, October). Retrieval of vertical leaf water content profile using terrestrial full-waveform lidar. In SPIE Remote Sensing (pp. 99981U-99981U). International Society for Optics and Photonics. 


\section{ITC Dissertation List}

https://www.itc.nl/Pub/research programme/Research-review-and-output/PhD-Graduates 